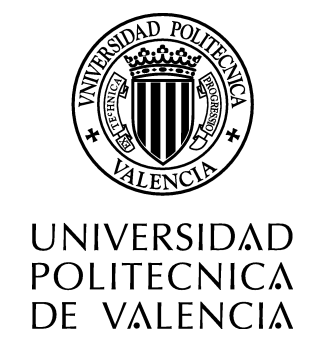

\title{
Contractive Maps and Complexity Analysis in Fuzzy Quasi-Metric Spaces
}

\author{
Memoria presentada por \\ Pedro Tirado Peláez \\ para optar al Grado de doctor en \\ Ciencias Matemáticas \\ Dirigida por el Doctor \\ D. Salvador Romaguera Bonilla
}

Valencia, Febrero 2008 

D. Salvador Romaguera Bonilla, Catedrático del Departamento de Matemática Aplicada de la Universidad Politécnica de Valencia

Certifico: que la presente memoria "Contractive Maps and Complexity analysis in Fuzzy Quasi-Metric Spaces" ha sido realizada bajo mi dirección por D. Pedro Tirado Peláez, en el Departamento de Matemática Aplicada de la Universidad Politécnica de Valencia, y constituye su tesis para optar al grado de Doctor en Ciencias Matemáticas.

Y para que así conste, presento la referida tesis, firmando el presente certificado.

Valencia, Febrero de 2008

Fdo. Salvador Romaguera Bonilla 


\section{Agradecimientos}

Parte de esta tesis ha sido elaborada en el marco del proyecto del Ministerio de educación y FEDER MTM2006-14925-C02-01.

Desde aquí quiero expresar mi más sincero agradecimiento al director de esta tesis, Salvador Romaguera. No es un tópico decir que gracias a él he podido realizar este trabajo. A lo largo de este periodo ha mostrado una enorme paciencia y dedicación, animándome en los momentos que más lo necesitaba. A su lado se puede aprender mucho, como científico y como persona. También quiero agradecer a Jesús Rodríguez su inestimable ayuda con latex. 
¡Qué agradable resulta caminar por la calle después de trabajar largo rato.! El mundo parece tan nuevo y sorprendente como si Dios lo hubiera creado ayer mismo. (Orhan Pamuk: Me llamo Rojo) 
$\mathrm{Al}$ último curso de acceso, sin que ellos sean conscientes, y a Mónica. 


\title{
Funciones Contractivas y Análisis de Complejidad en Espacios Casi-métricos Difusos
}

\author{
Pedro Tirado Peláez
}

En los últimos años se ha desarrollado una teoría matemática con propiedades robustas con el fin de fundamentar la Ciencia de la Computación. En este sentido, un avance significativo lo constituye el establecimiento de modelos matemáticos que miden la "distancia" entre programas y entre algoritmos, analizados según su complejidad computacional.

En 1995, M. Schellekens inició el desarrollo de un modelo matemático para el análisis de la complejidad algorítmica basado en la construcción de una casi-métrica definida en el espacio de las funciones de complejidad, proporcionando una interpretación computacional adecuada del hecho de que un programa o algoritmo sea más eficiente que otro en todos su "inputs". Esta información puede extraerse en virtud del carácter asimétrico del modelo. Sin embargo, esta estructura no es aplicable al análisis de algoritmos cuya complejidad depende de dos parámetros. Por tanto, en esta tesis introduciremos un nuevo espacio casi-métrico de complejidad que proporcionará un modelo útil para el análisis de este tipo de algoritmos. Por otra parte, el espacio casi-métrico de complejidad no da una interpretación computacional del hecho de que un programa o algoritmo sea sólo asintóticamente más eficiente que otro. Los espacios (casi-)métricos difusos aportan un parámetro "t", cuya adecuada utilización puede originar una información extra sobre el proceso computacional a estudiar; por ello introduciremos la noción de casi-métrica difusa de complejidad, que proporciona un modelo satisfactorio para interpretar la eficiencia asintótica de las funciones de complejidad.

En este contexto extenderemos los principales teoremas de punto fijo en espacios métricos difusos, utilizando una determinada noción de completitud, y obtendremos otros nuevos. Algunos de estos teoremas también se 
establecerán en el contexto general de los espacios casi-métricos difusos intuicionistas, de lo que resultarán condiciones de contracción menos fuertes.

Los resultados obtenidos se aplican a problemas interesantes en Ciencia de la Computación, como es la determinación de solución única para ecuaciones de recurrencia asociadas a determinados algoritmos, así como al análisis de la eficiencia asintótica de algoritmos. 


\title{
Funcions Contractives i Análisi de Complexitat en Espais Quasi-Mètrics Difusos
}

\author{
Pedro Tirado Peláez
}

En els últims anys s'ha desenrrollat una teoria matemàtica amb propietats robustes a fi de fonamentar la Ciència de la Computació. En este sentit, constituix un avaç significatiu l'establiment de models matemàtics que mesuren la distància entre programes i entre algoritmes, analitzats segons la complexitat computational respectiva.

En 1995, M. Schellekens va iniciar el desenrrollament d'un model matemàtic per a l'análisis de la complexitat algorímica basat en la construcció d'una quasi-métrica definida en l'espai de les funcions de complexitat, que proporcionava una intrepretació computacional adequada del fet que un programa o un algoritme siga més eficient que un atre en totes les entrades o inputs. Esta informació pot extraure's en virtut del caràcter asimètric del model. No obstante això, esta estructura no és aplicable a l'análisi d'algoritmes la complexitat dels quals depèn de dos paràmetres. Per tant, en esta tesi introduirem un nou espai quasi-mètric de complexitat que proporcionarà un model útil per a l'anàlisis d'aquest darrer tipus d'algoritmes. D'atra banda, l'espai quasi-mètric de complexitat no proporciona una interpretació computacional del fet que un programa o un algoritme siga només asimptòticament més eficient que un atre. Els espais (quasi-)mètrics difusos aporten un paràmetre "t", la utilització adecuada del qual pot aportar una informació extra sobre el procés computacional que es vol estudiar; per això introduirem la noció de quasi-mètrica difusa de complexitat, que proporciona un model satisfactori per a interpretar l'eficiència asimptòtica de les funcions de complexitat.

En este context estendrem els principals teoremes de punt fix en espais mètrics difusos, utilitzant una determinada noció de completitud, i n'obtendrem uns atres de nous. Alguns d'estos teoremes també s'establiran en el context general dels espais quasi-mètrics difusos intuicionistes, de la 
qual cosa resultaran unes condicions de contracció meyns fortes.

Els resultats obtinguts s'apliquen a problemes interessants en Ciència de la Computació, com ara la determinació de solució única per a equacions de recurrència associades a determinats algoritmes, així com a l'análisi de l'eficiència asimptòtica d'algoritmes. 


\title{
Contractive Maps and Complexity Analysis in Fuzzy Quasi-Metric Spaces
}

\author{
Pedro Tirado Peláez
}

In the last years a mathematical theory has been developed in order to obtain a foundation for Computing Science. In this setting an important progress is the establishment of mathematical models which are analyzed according to their computational complexity and measure the "distance" between programs and between algorithms.

In 1995, M. Schellekens started the development of a mathematical model to analyze the algorithmic complexity based on the construction of a quasimetric defined on the space of the complexity, providing an adequate computational interpretation of the fact that a program or an algorithm is more efficient than another in all of its inputs. This information can be extracted by the asymmetric nature of the model. However, this framework can not be applied to the analysis of those algorithms whose complexity depends on two parameters. Motivated by this interesting fact, in this thesis we will introduce a new complexity quasi-metric space which provides a useful model to analyze such algorithms. On the other hand, the complexity quasi-metric space does not give a computational interpretation of the fact that a program or an algorithm is only asymptotically more efficient than another. The fuzzy (quasi-)metric spaces provide a parameter " $t$ " such that a suitable use of this ingredient may give rise to extra information on the involved computational process; thus we will introduce the concept of complexity fuzzy quasi-metric space, which provides a successful model to interpret the asymptotic efficiency of the complexity functions.

In this context we will extend the main fixed-point theorems in fuzzy metric spaces, using an appropriate notion of completeness, and get new ones. Some of these theorems will be also established in the general framework of the intuitionistic fuzzy quasi-metric spaces, with less restrictive conditions of 
contraction.

From the obtained results we deduce a general method to identify the (unique) solution of recurrence equations associated to certain algorithms, as well as the analysis of asymptotic efficiency of such algorithms. 


\section{Contents}

1 Preliminaries and basic notions on fuzzy quasi-metric spaces 5

1.1 Introduction and basic notions . . . . . . . . . . . . . 5

2 Some remarks and examples on the topology of fuzzy metric spaces $\quad 19$

2.1 Introduction . . . . . . . . . . . . . . . . . . . . . . . . 19

2.2 Some basic results . . . . . . . . . . . . . . . . . . . . . 20

2.3 Examples on G-completeness and compactness . . . . . . . . . 22

3 Complexity analysis $\quad 33$

3.1 Introduction . . . . . . . . . . . . . . . . 33

3.2 The complexity space . . . . . . . . . . . . . . . 36

3.3 Recursive algorithms . . . . . . . . . . . . . . . . 37

3.4 Contraction maps on complexity spaces and expoDC algorithms 38

4 Application of fuzzy quasi-metrics to the theory of asymptotic complexity of algorithms 49

4.1 Introduction . . . . . . . . . . . . . . . . . 49

4.2 The complexity fuzzy quasi-metric space . . . . . . . . . . . 51

4.3 Fuzzy contractive maps and fixed point theorems . . . . . . . 54

4.4 Applications . . . . . . . . . . . . . . . . 57 
5 The Banach fixed point theorem in fuzzy quasi-metric spaces $\begin{array}{ll}\text { with application to the domain of words } & 61\end{array}$

5.1 Introduction . . . . . . . . . . . . . . . . . . 61

5.2 The Banach fixed point theorem in fuzzy quasi-metric spaces . 62

5.3 G-bicompleteness in non-Archimedean fuzzy quasi-metric spaces 64

5.4 Application to the domain of words . . . . . . . . . . 66

6 Contraction maps on fuzzy quasi-metric spaces and $[0,1]$ $\begin{array}{ll}\text { fuzzy posets } & 73\end{array}$

6.1 Introduction . . . . . . . . . . . . . . . 73

6.2 Fuzzy quasi-metric spaces and generalized [0,1]-fuzzy posets . 74

6.3 Contraction maps and fixed points . . . . . . . . . . 76

6.4 Application to recurrence equations . . . . . . . . . . . . . . 82

7 Some additional remarks on the fixed point theory for fuzzy (quasi-)metric spaces $\quad 85$

7.1 Introduction . . . . . . . . . . . . . . . 85

7.2 Contraction maps and fixed point theorems . . . . . . . 86

8 On fixed point theorems in intuitionistic fuzzy (quasi-)metric $\begin{array}{lr}\text { spaces } & 97\end{array}$

8.1 Introduction . . . . . . . . . . . . . . . . . . . 97

8.2 Intuitionistic fuzzy metric spaces and fixed point theorems . . 98

8.3 Co-fuzzy metric spaces . . . . . . . . . . . . 106

8.4 N-contractions and fixed point theorems . . . . . . . . . . 109

8.5 Intuitionistic fuzzy quasi-metric spaces (ifqm-spaces) and fixed point theorems . . . . . . . . . . . . . . . . 116

8.6 Application to recurrence equations of Quicksort . . . . . . . 127

9 Contraction maps on fuzzy complexity spaces and expoDC algorithms

9.1 Introduction . . . . . . . . . . . . . . . . . 131 
9.2 Complexity fuzzy quasi-metric spaces and expoDC algorithms 135 Bibliography 


\section{Chapter 1}

\section{Preliminaries and basic notions on fuzzy quasi-metric spaces}

\subsection{Introduction and basic notions}

The theory of fuzzy sets was introduced by Zadeh in 1965. From then, the research in many branches of fuzzy mathematics has received a great attention. In particular, and in the framework of fuzzy topology, one of the main problems in the theory of fuzzy topological spaces is to obtain an appropriate and consistent notion of a fuzzy metric space. Many authors have investigated this question and several different notions of a fuzzy metric space have been defined and studied. In [30], Kramosil and Michalek introduced and studied and interesting notion of fuzzy metric space which is closely related to a class of probabilistic metric spaces, the so-called (generalized) Menger spaces. By using the notion of a fuzzy metric space in the sense of Kramosil and Michalek [30], Grabiec proved in [21] fuzzy versions of the celebrated Banach fixed point theorem and of the Edelstein fixed point theorem, respectively. To this end, Grabiec introduced a notion of complete fuzzy metric space and of compact fuzzy metric space, respectively. Later on, George and Veeramani started in [19] the study of a stronger form of metric fuzziness. 
Further they modified the definition of Cauchy sequence given by Gabriec in [21], because of the fact that the set of real numbers is not complete with the definition given in [21].

On the other hand, it is well known that quasi-metric spaces constitute an efficient tool to discuss and solve several problems in topological algebra, approximation theory, theoretical computer science, etc.

In [22] Gregori and Romaguera introduced two notions of fuzzy quasimetric space that generalize the corresponding notions of fuzzy metric space by Kramosil and Michalek, and by George and Veeramani, to the quasimetric context.

Our basic reference for quasi-metric spaces is [17].

In the sequel the letters $\mathbb{R}, \mathbb{R}^{+}, \omega$ and $\mathbb{N}$ will denote the set of real numbers, the set of nonnegative real numbers, the set of nonnegative integer numbers and the set of positive integer numbers, respectively.

Following the modern terminology, by a quasi-metric on a nonempty set $X$ we mean a nonnegative real valued function $d$ on $X \times X$ such that for all $x, y, z \in X:$

(i) $x=y$ if and only if $d(x, y)=d(y, x)=0$;

(ii) $d(x, z) \leq d(x, y)+d(y, z)$.

If $d$ satisfies condition (i) above and

(ii') $d(x, z) \leq \max \{d(x, y), d(y, z)\}$

then, $d$ is called a non-Archimedean quasi-metric on $X$.

If $d$ satisfies the conditions (i), (ii) and

(ii") $d(x, y)=d(y, x)$

then, $d$ is called a metric on $X$. 
A (non-Archimedean) quasi-metric space is a pair $(X, d)$ such that $X$ is a nonempty set and $d$ is a (non-Archimedean) quasi-metric on $X$.

Each quasi-metric $d$ on $X$ generates a $T_{0}$ topology $\tau_{d}$ on $X$ which has as a base the family of open balls $\left\{B_{d}(x, r): x \in X, r>0\right\}$, where $B_{d}(x, r)=\{y \in X: d(x, y)<r\}$ for all $x \in X$ and $r>0$.

A topological space $(X, \tau)$ is said to be (quasi-)metrizable if there is a (quasi-)metric $d$ on $X$ such that $\tau=\tau_{d}$.

Given a (non-Archimedean) quasi-metric $d$ on $X$, then the function $d^{-1}$ defined on $X \times X$ by $d^{-1}(x, y)=d(y, x)$, is also a (non-Archimedean) quasimetric on $X$, called the conjugate of $d$, and the function $d^{s}$ defined on $X \times X$ by $d^{s}(x, y)=\max \left\{d(x, y), d^{-1}(x, y)\right\}$ is a (non-Archimedean) metric on $X$.

A quasi-metric space $(X, d)$ is said to be bicomplete if $\left(X, d^{s}\right)$ is a complete metric space. In this case, we say that $d$ is a bicomplete quasi-metric on $X$.

By a contraction map on a (quasi-)metric space $(X, d)$ we mean a selfmap $f$ on $X$ such that $d(f x, f y) \leq k d(x, y)$ for all $x, y \in X$, where $k$ is a constant with $0 \leq k<1$. The number $k$ is called a contraction constant for $f$.

It is clear that if $f$ is a contraction map on a quasi-metric space $(X, d)$ with contraction constant $k$, then $f$ is a contraction map on the metric space $\left(X, d^{s}\right)$ with contraction constant $k$.

According to [55], a binary operation $*:[0,1] \times[0,1] \rightarrow[0,1]$ is a continuous t-norm if $*$ satisfies the following conditions: $(\mathrm{i}) *$ is associative and commutative; (ii) $*$ is continuous; (iii) $a * 1=a$ for every $a \in[0,1]$; (iv) 
$a * b \leq c * d$ whenever $a \leq c$ and $b \leq d$, with $a, b, c, d \in[0,1]$.

It is easy to see that the following statements hold:

(1) If $0 \leq r<s \leq 1$, then $r * t<s$ for all $t \in[0,1]$.

(2) If $1>r>s \geq 0$, there exists $t \in(s, 1)$ such that $r * t \geq s$.

(3) If $1>r \geq 0$, there exists $t \in(r, 1)$ such that $t * t \geq r$.

Paradigmatic examples of continuous t-norm are Min, Prod, and $T_{L}$ (the Lukasiewicz t-norm).

In the following Min will be denoted by $\wedge$, Prod by $\cdot$ and $T_{L}$ by $*_{L}$. Thus we have $a \wedge b=\min \{a, b\}, a \operatorname{Prod} b=a . b$ and $a *_{L} b=\max \{a+b-1,0\}$ for all $a, b \in[0,1]$. The following relations hold:

$\wedge \geqslant \cdot \geqslant *_{L}$. In fact, $\wedge \geqslant *$ for any continuous t-norm $*$.

Similarly, a binary operation $\diamond:[0,1] \times[0,1] \rightarrow[0,1]$ is a continuous tconorm if $\diamond$ satisfies the following conditions: (i) $\diamond$ is associative and commutative; (ii) $\diamond$ is continuous; (iii) $a \diamond 0=a$ for every $a \in[0,1]$; (iv) $a \diamond b \leq c \diamond d$ whenever $a \leq c$ and $b \leq d$, with $a, b, c, d \in[0,1]$.

It is easy to see that the following statements hold:

(1) If $1 \geq r>s \geq 0$, then $r \diamond t>s$ for all $t \in[0,1]$.

(2) If $0<r<s \leq 1$, there exists $t \in(0, s)$ such that $r \diamond t \leq s$.

(3) If $0<r \leq 1$, there exists $t \in(0, r)$ such that $t \diamond t \leq r$.

If $*$ is any continuous t-norm we can define a continuous t-conorm $\diamond$ as follows: 


$$
a \diamond b=1-[(1-a) *(1-b)] \text { for all } a, b \in[0,1]
$$

This continuous t-conorm $\diamond$ is known as the continuous t-conorm associated to the continuous t-norm $*$.

Examples of continuous t-conorm are Max, which is the continuous tconorms associated to $\wedge$, and the the continuous t-conorms associated to - and $*_{L}$ respectively. In the following these continuous t-conorms will be denoted by $\vee, \diamond_{P}$ and $\nabla_{L}$, respectively. In particular we have $a \diamond_{L} b=$ $\min \{1, a+b\}$. The continuous t-conorm $\diamond_{L}$ will be called the Lukasiewicz t-conorm.

It is well known, and easy to see, that if $*$ is a continuous t-norm and $\diamond$ is a continuous t-conorm, then for all $a, b, c, d \in[0,1]$ :

$$
a * b \leq a \wedge b \leq a \vee b \leq a \diamond b
$$

Examples of classes of continuous t-norm and continuous t-conorm ([8]), that cover the full ranges of these operations, are defined for all $a, b \in[0,1]$ by:

$$
\left\{\begin{array}{c}
a *_{\alpha} b=1-\min \left\{1,\left[(1-a)^{1 / \alpha}+(1-b)^{1 / \alpha}\right]^{\alpha}\right\} \\
a \diamond_{\alpha} b=\min \left\{1,\left(a^{1 / \alpha}+b^{1 / \alpha}\right)^{\alpha}\right\}
\end{array}\right.
$$

where $\alpha$ is a parameter whose range is $(0, \infty)$. A particular continuous tnorm and one particular continuous t-conorm are obtained for each value of the parameter $\alpha$. These operations are often referred to in the literature as the Yager continuous t-norm and continuous t-conorm. 
It is easy to see that $a *_{\alpha_{1}} b \geq a *_{\alpha_{2}} b$ and $a \diamond_{\alpha_{1}} b \leq a \diamond_{\alpha_{2}} b$ whenever $\alpha_{1} \leq \alpha_{2}$, with $a, b \in[0,1]$. In particular $a *_{n_{1}} b \geq a *_{n_{2}} b$ and $a \diamond_{n_{1}} b \leq a \diamond_{n_{2}} b$ whenever $n_{1} \leq n_{2}$, with $n_{1}, n_{2} \in \mathbb{N}$ and $a, b \in[0,1]$.

A subclass of Yager continuous t-norm and continuous t-conorm is $\left\{*_{\alpha}\right\}_{\alpha \in \mathbb{N}}$ and $\left\{\diamond_{\alpha}\right\}_{\alpha \in \mathbb{N}}$. In particular we have that $*_{1}$ and $\nabla_{1}$ are the Lukasiewicz tnorm and the Lukasiewicz t-conorm respectively. We will call these subclasses as the $\mathbb{N}$-Yager continuous t-norm and continuous t-conorm, respectively.

Definition 1.1 [24]. A KM-fuzzy quasi-metric on a set $X$ is a pair $(M, *)$ such that $*$ is a continuous t-norm and $M$ is a fuzzy set in $X \times X \times[0, \infty)$ such that for all $x, y, z \in X$ :

(KM1) $M(x, y, 0)=0$;

(KM2) $x=y$ if and only if $M(x, y, t)=M(y, x, t)=1$ for all $t>0$;

(KM3) $M(x, z, t+s) \geq M(x, y, t) * M(y, z, s)$ for all $t, s \geq 0$;

$(\mathrm{KM} 4) M\left(x, y,_{-}\right):[0, \infty) \rightarrow[0,1]$ is left continuous.

Note that a KM-fuzzy quasi-metric $(M, *)$ satisfying for all $x, y \in X$ and $t>0$ the symmetry axiom $M(x, y, t)=M(y, x, t)$, is a fuzzy metric in the sense of Kramosil and Michalek [30].

Definition 1.2 [24]. A KM-fuzzy quasi-metric space is a triple $(X, M, *)$ such that $X$ is a (nonempty) set and $(M, *)$ is a KM-fuzzy quasi-metric on $X$.

If $(M, *)$ is a fuzzy metric in the sense of Kramosil and Michalek then $(X, M, *)$ is a fuzzy metric space in the sense of Kramosil and Michalek [30].

In the following, KM-fuzzy quasi-metrics and fuzzy metrics in the sense of Kramosil and Michalek will be simply called fuzzy quasi-metrics and fuzzy 
metrics respectively, and KM-fuzzy quasi-metric spaces and fuzzy metric spaces in the sense of Kramosil and Michalek will be simply called fuzzy quasi-metric spaces and fuzzy metric spaces, respectively.

It was shown in Proposition 1 of [24] that if $(M, *)$ is a fuzzy quasi-metric on $X$, then for each $x, y \in X, M\left(x, y,_{-}\right)$is nondecreasing, i.e. $M(x, y, t) \leq$ $M(x, y, s)$ whenever $t \leq s$.

If $(M, *)$ is a fuzzy quasi-metric on $X$, then $\left(M^{-1}, *\right)$ is also a fuzzy quasi-metric on $X$, where $M^{-1}$ is the fuzzy set in $X \times X \times[0, \infty)$ defined by $M^{-1}(x, y, t)=M(y, x, t)$. Moreover, if we denote by $M^{i}$ the fuzzy set in $X \times X \times[0, \infty)$ given by $M^{i}(x, y, t)=\min \left\{M(x, y, t), M^{-1}(x, y, t)\right\}$, then $\left(M^{i}, *\right)$ is a fuzzy metric on $X[24]$.

Given a fuzzy quasi-metric space $(X, M, *)$ we define the open ball $B_{M}(x, r, t)$, for $x \in X, 0<r<1$, and $t>0$, as the set $B_{M}(x, r, t)=\{y \in X$ : $M(x, y, t)>1-r\}$. Obviously, $x \in B_{M}(x, r, t)$.

For each $x \in X, 0<r_{1} \leq r_{2}<1$ and $0<t_{1} \leq t_{2}$, we have $B_{M}\left(x, r_{1}, t_{1}\right) \subseteq$ $B_{M}\left(x, r_{2}, t_{2}\right)$. Consequently, we may define a topology $\tau_{M}$ on $X$ as

$\tau_{M}:=\left\{A \subseteq X:\right.$ for each $x \in A$ there are $r \in(0,1), t>0$, with $\left.B_{M}(x, r, t) \subseteq A\right\}$

Moreover, for each $x \in X$ the collection of open balls $\left\{B_{M}(x, 1 / n, 1 / n)\right.$ : $n=2,3 \ldots\}$, is a local base at $x$ with respect to $\tau_{M}$. It is clear, that for any fuzzy quasi-metric space $(X, M, *), \tau_{M}$ is a $T_{0}$ topology.

The topology $\tau_{M}$ is called the topology generated by the fuzzy quasimetric space $(X, M, *)$. It is clear that each open ball $B_{M}(x, r, t)$ is an open set for the topology $\tau_{M}$. 
Definition 1.3 [24]. A GV-fuzzy quasi-metric on a set $X$ is a pair $(M, *)$ such that $*$ is a continuous t-norm and $M$ is a fuzzy set in $X \times X \times(0, \infty)$ such that for all $x, y, z \in X ; t, s>0$ :

(GV1) $M(x, y, t)>0$;

(GV2) $x=y$ if and only if $M(x, y, t)=M(y, x, t)=1$;

(GV3) $M(x, z, t+s) \geq M(x, y, t) * M(y, z, s)$;

$(\mathrm{GV} 4) M\left(x, y,{ }_{-}\right):(0, \infty) \rightarrow(0,1]$ is continuous.

Note that a GV-fuzzy quasi-metric $(M, *)$ satisfying for all $x, y \in X$ and $t>0$ the symmetry axiom $M(x, y, t)=M(y, x, t)$, is a fuzzy metric in the sense of George and Veeramani [19] (in the following a GV-fuzzy metric).

Definition 1.4 [24]. A GV-fuzzy quasi-metric space is a triple $(X, M, *)$ such that $X$ is a (nonempty) set and $(M, *)$ is a $G V$-fuzzy quasi-metric on $X$.

If $(M, *)$ is a $\mathrm{GV}$-fuzzy metric then $(X, M, *)$ is a fuzzy metric space in the sense of George and Veeramani [19] (in the following a GV-fuzzy metric space).

If $(M, *)$ is a GV-fuzzy quasi-metric on $X$, then $\left(M^{-1}, *\right)$ is also a GVfuzzy quasi-metric on $X$, where $M^{-1}$ is the fuzzy set in $X \times X \times(0, \infty)$ defined by $M^{-1}(x, y, t)=M(y, x, t)$. Moreover, if we denote by $M^{i}$ the fuzzy set in $X \times X \times(0, \infty)$ given by $M^{i}(x, y, t)=\min \left\{M(x, y, t), M^{-1}(x, y, t)\right\}$, then $\left(M^{i}, *\right)$ is a GV-fuzzy metric on $X[24]$.

Obviously, each GV-fuzzy (quasi-)metric $(M, *)$ can be considered as a fuzzy (quasi-)metric by defining $M(x, y, 0)=0$ for all $x, y \in X$. Therefore, each GV-fuzzy (quasi-)metric space generates a topology $\tau_{M}$ defined as in the KM-case; thus if $(X, M, *)$ is a $\mathrm{GV}$-fuzzy (quasi-)metric space we have 
that for each $x, y \in X, M\left(x, y,_{-}\right)$is nondecreasing and that each open ball is an open set for the topology $\tau_{M}$.

We say that a topological space $(X, \tau)$ admits a compatible $(\mathrm{GV}$-)fuzzy (quasi-)metric if there is a (GV-)fuzzy (quasi-)metric $(M, *)$ on $X$ such that $\tau=\tau_{M}$.

Gregori and Romaguera showed in [22] and [24] that every (quasi-)metric induces a fuzzy (quasi-)metric and, conversely, every fuzzy (quasi-)metric space generates a (quasi-)metrizable topology. (See Example 1.1 and Theorem 1.1, below).

Proposition 1.1. [24, 19]. A sequence $\left\{x_{n}\right\}_{n}$ in a fuzzy (quasi-)metric space $(X, M, *)$ converges to a point $x \in X$ with respect to $\tau_{M}$ if and only if $\lim _{n} M\left(x, x_{n}, t\right)=1$, for all $t>0$.

Example $1.1[24,19]$. Let $(X, d)$ be a (quasi-)metric space, let $*$ be any continuous t-norm and let $M_{d}$ be the function defined on $X \times X \times(0, \infty)$ by

$$
M_{d}(x, y, t)=\frac{t}{t+d(x, y)}
$$

Then $\left(X, M_{d}, *\right)$ is a $G V$-fuzzy (quasi-)metric space called standard $G V$ fuzzy (quasi-)metric space and $\left(M_{d}, *\right)$ is the fuzzy (quasi-)metric induced by d. Furthermore, it is easy to check that $\left(M_{d}\right)^{-1}=M_{d^{-1}}$ and $\left(M_{d}\right)^{i}=M_{d^{s}}$, and the topology $\tau_{d}$, generates by $d$, coincides with the topology $\tau_{M_{d}}$ generated by the induced GV-fuzzy (quasi-)metric $(M, *)$.

Theorem $1.1[22,24]$. For a topological space $(X, \tau)$ the following are equivalent.

(1) $(X, \tau)$ is (quasi-)metrizable.

(2) $(X, \tau)$ admits a compatible GV-fuzzy (quasi-)metric. 
(3) $(X, \tau)$ admits a compatible fuzzy (quasi-)metric.

As we indicated above, by using the notion of a fuzzy metric space in the sense of Kramosil and Michalek [30], Grabiec proved in [21] fuzzy versions of the celebrated Banach fixed point theorem and Edelstein fixed point theorem respectively. To this end, Grabiec introduced a notion of complete fuzzy metric space and of compact fuzzy metric space respectively. Next we present the definitions and theorems given by Grabiec in [21]. These notions will be termed as G-Cauchy sequence and G-complete, respectively.

Definition 1.5 [21]. A sequence $\left\{x_{n}\right\}_{n}$ in a fuzzy metric space $(X, M, *)$ is called G-Cauchy if $\lim _{n \rightarrow \infty} M\left(x_{n}, x_{n+p}, t\right)=1$ for each $t>0$ and $p \in \mathbb{N}$.

Note that $\left\{x_{n}\right\}_{n}$ is a G-Cauchy sequence if and only if for each $\varepsilon \in$ $(0,1), p \in \mathbb{N}, t>0$ there exists $n_{0} \in \mathbb{N}$ such that $M\left(x_{n}, x_{n+p}, t\right)>1-\varepsilon$ for all $n \geqslant n_{0}$.

Definition 1.6 [21]. A fuzzy metric space $(X, M, *)$ is called G-complete if every G-Cauchy sequence in $X$ is convergent with respect to $\tau_{M}$. In this case, $(M, *)$ is called a $G$-complete fuzzy metric on $X$.

Theorem 1.2 [21]. Let $(X, M, *)$ be a G-complete fuzzy metric space such that $\lim _{t \rightarrow \infty} M(x, y, t)=1$ for all $x, y \in X$. Let $T: X \rightarrow X$ be a self-map satisfying:

$$
M(T x, T y, k t) \geq M(x, y, t)
$$

for all $x, y \in X$, and $t>0$, with $k \in(0,1)$. Then $T$ has a unique fixed point.

By Theorem 1.1, the topology $\tau_{M}$ generated by a fuzzy metric space $(X, M, *)$ is a metrizable topology. Following [22], a fuzzy metric space 
$(X, M, *)$ is called compact if $\left(X, \tau_{M}\right)$ is a compact topological space. Therefore a definition of compact fuzzy metric space can be equivalently given as follows:

Definition 1.7 [21]. A fuzzy metric space $(X, M, *)$ is called compact if every sequence has a convergent subsequence.

Theorem $1.3[21]$. Let $(X, M, *)$ be a compact fuzzy metric space and let $T: X \rightarrow X$ be a self-map satisfying:

$$
M(T x, T y, t)>M(x, y, t)
$$

for all $x, y \in X$ such that $x \neq y$, and $t>0$. Then $T$ has a unique fixed point.

George and Veeramani gave in [19] the following example which shows that with Grabiec's notion of completeness, $\mathbb{R}$ fails to be complete.

Example 1.2 [19]. For $x, y \in \mathbb{R}, t>0$, define

$$
M_{d}(x, y, t)=\frac{t}{t+d(x, y)}
$$

( $d$ is the Euclidean metric on $\mathbb{R}$ ).

Then $\left(M_{d}, *\right)$ is the standard $G V$-fuzzy metric induced by $d$ on $\mathbb{R}$.

Let $s_{n}=1+\frac{1}{2}+\frac{1}{3}+\cdots+\frac{1}{n}$, for $n \in \mathbb{N}$.

Thus

$$
M_{d}\left(s_{n+p}, s_{n}, t\right)=\frac{t}{t+1 /(n+1)+\cdots+1 /(n+p)},
$$

SO

$$
\lim _{n} M_{d}\left(s_{n+p}, s_{n}, t\right)=1,
$$

for all $p \in \mathbb{N}$ and $t>0$. 
Thus $\left\{s_{n}\right\}_{n}$ is a G-Cauchy sequence in $\left(\mathbb{R}, M_{d}, *\right)$.

It is well known that $\left\{s_{n}\right\}_{n}$ is not convergent, therefore $\left(\mathbb{R}, M_{d}, *\right)$ fails to be G-complete.

Because of this fact, George and Veeramani modified the definitions of Cauchy sequence and completeness due to Grabiec, as follows:

Definition 1.8 [19]. A sequence $\left\{x_{n}\right\}_{n}$ in a fuzzy metric space $(X, M, *)$ is called a Cauchy sequence if for each $\varepsilon \in(0,1), t>0$ there exists $n_{0} \in \mathbb{N}$ such that $M\left(x_{n}, x_{m}, t\right)>1-\varepsilon$ for all $n, m \geqslant n_{0}$.

Definition 1.9 [19]. A fuzzy metric space $(X, M, *)$ is called complete if every Cauchy sequence is convergent with respect to $\tau_{M}$.

It follows from Definition 1.9 that $\left(\mathbb{R}, M_{d}, *\right)$ is a complete GV-fuzzy quasi-metric space.

Next we present the generalization of the above definitions to the fuzzy quasi-metric setting.

Definition 1.10. A sequence $\left\{x_{n}\right\}_{n}$ in a fuzzy quasi-metric space $(X, M, *)$ is called G-Cauchy if $\left\{x_{n}\right\}_{n}$ is a G-Cauchy sequence in $\left(X, M^{i}, *\right)$.

Definition 1.11. A fuzzy quasi-metric space $(X, M, *)$ is called G-bicomplete if $\left(X, M^{i}, *\right)$ is a $G$-complete fuzzy metric space. In this case, $(M, *)$ is called a G-bicomplete fuzzy quasi-metric on $X$.

It follows from the preceding definitions that a fuzzy quasi-metric space $(X, M, *)$ is G-bicomplete if and only if every G-Cauchy sequence converges with respect to $\tau_{M^{i}}$. 
Definition 1.12 (compare [19]). A sequence $\left\{x_{n}\right\}_{n}$ in a fuzzy quasi-metric space $(X, M, *)$ is called a Cauchy sequence if for each $\varepsilon \in(0,1), t>0$, there exists $n_{0} \in \mathbb{N}$ such that $M\left(x_{n}, x_{m}, t\right)>1-\varepsilon$ for all $n, m \geqslant n_{0}$.

Definition 1.13. A fuzzy quasi-metric space $(X, M, *)$ is called bicomplete if $\left(X, M^{i}, *\right)$ is a complete fuzzy metric space. In this case, $(M, *)$ is called a bicomplete fuzzy quasi-metric on $X$.

It follows from the preceding definitions that a fuzzy quasi-metric space $(X, M, *)$ is bicomplete if and only if every Cauchy sequence converges with respect to $\tau_{M^{i}}$.

A metrizable topological space $(X, \tau)$ is said to be completely metrizable if it admits a compatible complete metric. It was proved in [22] that a topological space is completely metrizable if and only if it admits a compatible complete fuzzy metric.

Proposition $1.2[24]$. (a) Let $(X, M, *)$ be a bicomplete fuzzy quasi-metric space. Then $\left(X, \tau_{M}\right)$ admits a compatible bicomplete quasi-metric.

(b) Let $(X, d)$ be a bicomplete quasi-metric space. Then $\left(X, M_{d}, *\right)$ is a bicomplete $G V$-fuzzy quasi-metric space. 


\section{Chapter 2}

\section{Some remarks and examples on the topology of fuzzy metric spaces}

\section{$2.1 \quad$ Introduction}

In this chapter we give some results on the topology of fuzzy metric spaces in the sense of Kramosil and Michalek. In particular we present an example of a closed ball that is not a closed set in a fuzzy metric space. On the other hand, we observe that the open sets of this topology can be defined by means of open balls with $t \in(0, \varepsilon)$ and $\varepsilon>0$. From this fact we give a generalized version of Grabiec's version of the Edelstein fixed point theorem. Finally we give a new example of a complete fuzzy metric space which is not G-complete and two essentially different examples of compact fuzzy metric spaces that are not G-complete. These results are contained in [58]. 


\subsection{Some basic results}

Given a fuzzy (quasi-)metric space $(X, M, *)$ we define the closed ball $\bar{B}_{M}(x, r, t)$, for $x \in X, 0<r<1$, and $t>0$, as the set $\bar{B}_{M}(x, r, t)=\{y \in X$ : $M(x, y, t) \geq 1-r\}$. Obviously, $x \in \bar{B}_{M}(x, r, t)$.

George and Veeramani proved in [19] that every closed ball is a closed set in a GV-fuzzy metric space. Nevertheless it is not true in a fuzzy metric space in the sense of Kramosil and Michalek as the following example shows.

Example 2.1. Let $(X, d)$ be the metric space where $X=[0,1]$ and $d$ is the Euclidean metric on $X$. In [35] it is shown that $(X, M, *)$ is a fuzzy metric space, where $*$ is any continuous t-norm and $M$ is the fuzzy set in $X \times X \times[0,+\infty)$ given in the following way:

$$
\begin{aligned}
& M(x, y, t)=1, \text { if } d(x, y)<t \\
& M(x, y, t)=0, \text { if } d(x, y) \geq t
\end{aligned}
$$

We will show that there exists a closed ball $\bar{B}_{M}(x, r, t)$, and a point $z \in X$ such that $z \in \overline{\bar{B}}_{M}(x, r, t) \backslash \bar{B}_{M}(x, r, t)$.

Let $\overline{B_{M}}(0,1 / 2,1)=\{y: M(0, y, 1) \geqslant 1 / 2\}$. According to the definition of $M(x, y, t)$ we deduce that $\overline{B_{M}}(0,1 / 2,1)=\{y: M(0, y, 1)=1\}=\{y: d(0, y)<1\}=$ $[0,1)$.

Let $\left\{x_{n}\right\}_{n}$ be the sequence in $(X, M, *)$, where $x_{n}=1-\frac{1}{n}$, for all $n \in \mathbb{N}$. Obviously this sequence converges to $z=1$. On the other hand we have that $x_{n} \in \overline{B_{M}}(0,1 / 2,1)$ for all $n \in \mathbb{N}$. So, for $z=1$ we have that $z \in \overline{\overline{B_{M}}}(0,1 / 2,1)$, and nevertheless $z \notin \overline{B_{M}}(0,1 / 2,1)$.

Remark 2.1. Fix $\varepsilon \in(0,1)$. Given $t \in(0, \varepsilon)$ there exists $n \in \mathbb{N}$ such that $1 / n<t$, so $B_{M}(x, r, 1 / n) \subseteq B_{M}(x, r, t)$ for all $r \in(0,1)$. On the other hand 
given $n \in \mathbb{N}$ there exists $t \in(0, \varepsilon)$ such that $B_{M}(x, r, t) \subseteq B_{M}(x, r, 1 / n)$. Therefore the collection $\left\{B_{M}(x, r, t): t \in(0, \varepsilon)\right\}$ is a base for the topology $\tau_{M}$.

We consider the above observation to generalize the fuzzy metric version of fixed point theorem of Edelstein given by Grabiec (Theorem 1.3). Next we show an example where Theorem 1.3 can not be applied.

Example 2.2. Let $(X, d)$ be the metric space where $X=[0,1]$ and $d$ the Euclidean metric on $X$. Let $*_{L}$ be the Lukasiewicz continuous t-norm. We define a fuzzy set $M$ in $X \times X \times[0,+\infty)$ given in the following way:

$$
\begin{aligned}
M(x, y, 0) & =0, \\
M(x, y, t) & =1-d(x, y), \text { if } 0<t \leqslant 1, \\
M(x, y, t) & =1, \text { if } t>1 .
\end{aligned}
$$

It is clear that $\left(X, M, *_{L}\right)$ is a compact fuzzy metric space.

Let $T: X \rightarrow X$ be given by $T x=x / 2$ for all $x \in X$ (obviously $T$ has a unique fixed point $x=0)$. Nevertheless the conditions of Theorem 1.3 are not satisfied because $M(T x, T y, t)=M(x, y, t)=1$ for all $t>1$, and for all $x, y \in X$.

Since the topology $\tau_{M}$ can be defined by means of open balls with $t \in$ $(0, \varepsilon), \varepsilon \in(0,1)$, and for each $x, y \in X, M\left(x, y,{ }_{-}\right)$is nondecreasing, following the proof of Edelstein 's theorem given in [21] we can generalize the fuzzy metric version of the fixed point theorem of Edelstein given by Grabiec as follows:

Theorem 2.1. Let $(X, M, *)$ be a compact fuzzy metric space and let $T$ : $X \rightarrow X$ be a self-map satisfying: 


$$
M(T x, T y, t)>M(x, y, t)
$$

for all $x, y \in X$ such that $x \neq y$, and for all $t \in(0, \varepsilon)$, with $\varepsilon>0$. Then $T$ has a unique fixed point.

Observe that the conditions of Theorem 2.1 are satisfied in Example 2.2.

\subsection{Examples on G-completeness and com- pactness}

We start this section giving the corresponding notions of G-Cauchyness and G-completeness for metric spaces.

Definition 2.2 A sequence $\left\{x_{n}\right\}_{n}$ in a metric space $(X, d)$ is called $G$ Cauchy sequence if $\lim _{n \rightarrow \infty} d\left(x_{n}, x_{n+p}\right)=0$ for each $p \in \mathbb{N}$.

Definition 2.3 A metric space $(X, d)$ is called $G$-complete if every $G$-Cauchy sequence in $X$ is convergent with respect to $\tau_{d}$. In this case, $d$ is called a $G$ complete metric on $X$.

Let $(X, d)$ be a metric space and let $\left(X, M_{d}, *\right)$ be the standard fuzzy metric space. Recall (Example 1.1) that the topology $\tau_{d}$, generated by $d$, coincides with the topology $\tau_{M_{d}}$. Furthermore a sequence $\left\{x_{n}\right\}_{n}$ in $X$ is a Cauchy sequence in $(X, d)$ if and only if $\left\{x_{n}\right\}_{n}$ is a Cauchy sequence in $\left(X, M_{d}, *\right)$. Indeed, let $\left\{x_{n}\right\}_{n}$ be a Cauchy sequence in $(X, d)$. Fix $t>0$. Let $\varepsilon \in(0,1)$ such that $t>1-\varepsilon$. There exists $n_{0} \in \mathbb{N}$ such that $d\left(x_{n}, x_{m}\right)<\varepsilon$ for all $m, n \geq n_{0}$. Therefore $M\left(x_{n}, x_{m}, t\right)=t /\left(t+d\left(x_{n}, x_{m}\right)\right)>t /(t+\varepsilon)>1-\varepsilon$ for all $m, n \geq n_{0}$. So $\left\{x_{n}\right\}$ is a Cauchy sequence in $\left(X, M_{d}, *\right)$. Conversely, if $\left\{x_{n}\right\}_{n}$ is a Cauchy 
sequence in $\left(X, M_{d}, *\right)$, given $\varepsilon \in(0,1 / 2)$ there exists $n_{0} \in \mathbb{N}$ such that $M\left(x_{n}, x_{m}, 1\right)>1-\varepsilon$ for all $m, n \geq n_{0}$. So $1 /\left(1+d\left(x_{n}, x_{m}\right)\right)>1-\varepsilon$ for all $m, n \geq n_{0}$, thus $d\left(x_{n}, x_{m}\right)<\varepsilon /(1-\varepsilon)<2 \varepsilon$ for all $m, n \geq n_{0}$. We conclude that $\left\{x_{n}\right\}_{n}$ is a Cauchy sequence in $(X, d)$. Therefore $\left(X, M_{d}, *\right)$ is a complete GV-fuzzy metric space if and only if $(X, d)$ is a complete metric space.

In the same way we obtain that a sequence $\left\{x_{n}\right\}_{n}$ in $X$ is a G-Cauchy sequence in $(X, d)$ if and only if $\left\{x_{n}\right\}_{n}$ is a G-Cauchy sequence in $\left(X, M_{d}, *\right)$ and that $\left(X, M_{d}, *\right)$ is a $\mathrm{G}$-complete fuzzy metric space if and only if $(X, d)$ is a $\mathrm{G}$-complete metric space.

George and Veeramani showed (Example 1.2) that with Grabiec's notion of completeness, $\mathbb{R}$ fails to be complete. Next we give another example of a complete fuzzy metric space which is not G-complete.

Example 2.3. Let $\mathcal{C}([0,1])$ be the set of all continuous functions from $[0,1]$ into itself and let $d_{s}$ be the metric on $\mathcal{C}([0,1])$ given by $d_{s}(f, g)=$ $\sup _{x \in[0,1]}|f(x)-g(x)|$. It is known that $\left(\mathcal{C}([0,1]), d_{s}\right)$ is a complete metric space, therefore $\left(\mathcal{C}([0,1]), M_{d_{s}}, *\right)$ is a complete fuzzy metric space.

Let $\left\{f_{n}\right\}_{n}$ be the sequence in $\left(\mathcal{C}([0,1]), M_{d_{s}}, \cdot\right)$ given by $f_{n}(x)=x^{n}$. It is well known that $\left\{f_{n}\right\}_{n}$ is a non-convergent sequence in $\left(\mathcal{C}([0,1]), d_{s}\right)$, therefore $\left\{f_{n}\right\}_{n}$ is a non-convergent sequence in $\left(\mathcal{C}([0,1]), M_{d_{s}}, *\right)$. We will show that $\left\{f_{n}\right\}_{n}$ is a G-Cauchy sequence in $\left(\mathcal{C}([0,1]), d_{s}\right)$, and hence it is a nonconvergent $G$-Cauchy sequence in $\left(\mathcal{C}([0,1]), M_{d_{s}}, *\right)$, so that $\left(\mathcal{C}([0,1]), M_{d_{s}}, *\right)$ is not G-complete.

Indeed, let $p \in \mathbb{N}$, then:

$$
d\left(f_{n}, f_{n+p}\right)=\sup _{x \in[0,1]}\left|x^{n}-x^{n+p}\right|=\max _{x \in[0,1]}\left|x^{n}-x^{n+p}\right|,
$$

An easy computation, based in optimization, shows that this maximum is 
obtained in the point $x=\left(\frac{n}{n+p}\right)^{1 / p}$, thus:

$$
\lim _{n \rightarrow \infty} d\left(f_{n}, f_{n+p}\right)=\lim _{n \rightarrow \infty} \max _{x \in[0,1]}\left|x^{n}-x^{n+p}\right|=\lim _{n \rightarrow \infty}\left(\frac{n}{n+p}\right)^{n / p}\left(1-\frac{n}{n+p}\right),
$$

so

$$
\lim _{n \rightarrow \infty} d\left(f_{n}, f_{n+p}\right)=0 .
$$

Thus $\left\{f_{n}\right\}_{n}$ is a G-Cauchy sequence in $\left(\mathcal{C}([0,1]), d_{s}\right)$. We conclude that $\left(\mathcal{C}([0,1]), M_{d_{s}}, *\right)$ is not $G$-complete.

Next we show that G-completeness is a very strong kind of completeness. In fact, we here present two essentially different examples of compact fuzzy metric spaces that are not complete in Grabiec's sense.

Gregori and Romaguera obtained the following theorem:

Theorem 2.2 [22]. A fuzzy metric space is compact if and only if it is precompact and complete.

Hence every compact fuzzy metric space is complete (in George and Veeramani's sense).

Example 2.4. Let $X=[0,1 / 2] \times[0,1 / 2]$. Obviously $(X, d)$ is a compact metric space, where $d$ is the metric on $X$ given by $d(x, y)=\left|y_{1}-x_{1}\right|+$ $\left|y_{2}-x_{2}\right|$, with $x=\left(x_{1}, x_{2}\right)$ and $y=\left(y_{1}, y_{2}\right)$. Therefore $\left(X, M_{d}, *\right)$ is a compact fuzzy metric space.

Construct a sequence $\left\{x_{m}\right\}_{m \geqslant 2}$, in $\left(X, M_{d}, *\right)$ as follows:

Given $m \geqslant 2$, we have: 
i) $m=2^{2 n_{m}-1}+j$ for some $n_{m} \in \mathbb{N}$ and some $j \in w$ with $0 \leqslant j \leqslant 2^{2 n_{m}-1}$, or

ii) $m=2^{2 n_{m}}+j$ for some $n_{m} \in \mathbb{N}$ and some $j \in w$ with $0 \leqslant j \leqslant 2^{2 n_{m}}$.

In case i) put:

$$
x_{m}=\left(\frac{m-2^{2 n_{m}-1}}{2^{2 n_{m}}}, \frac{1}{2^{2 n_{m}-1}}-\frac{m-2^{2 n_{m}-1}}{2^{2 n_{m}} 2^{2 n_{m}-1}}\right) .
$$

In case ii) put:

$$
x_{m}=\left(\frac{2^{2 n_{m}+1}-m}{2^{2 n_{m}+1}}, \frac{1}{2^{2 n_{m}}}-\frac{m-2^{2 n_{m}}}{2^{2 n_{m}+1} 2^{2 n_{m}}}\right) .
$$

It is easy to see (see Figure 2.1) that $\left\{x_{2^{2 n-1}}\right\}_{n}$ and $\left\{x_{2^{2 n}}\right\}_{n}$ are subsequences of $\left\{x_{m}\right\}_{m \geqslant 2}$ where $x_{2^{2 n-1}}=\left(0,1 / 2^{2 n-1}\right)$ and $x_{2^{2 n}}=\left(1 / 2,1 / 2^{2 n}\right)$, $n \in \mathbb{N}$ (see figure 2.1). We have that $\lim _{n} x_{2^{2 n-1}}=(0,0)$ and $\lim _{n} x_{2^{2 n}}=$ $(1 / 2,0)$, and thus $\left\{x_{m}\right\}_{m \geqslant 2}$ is not convergent. 


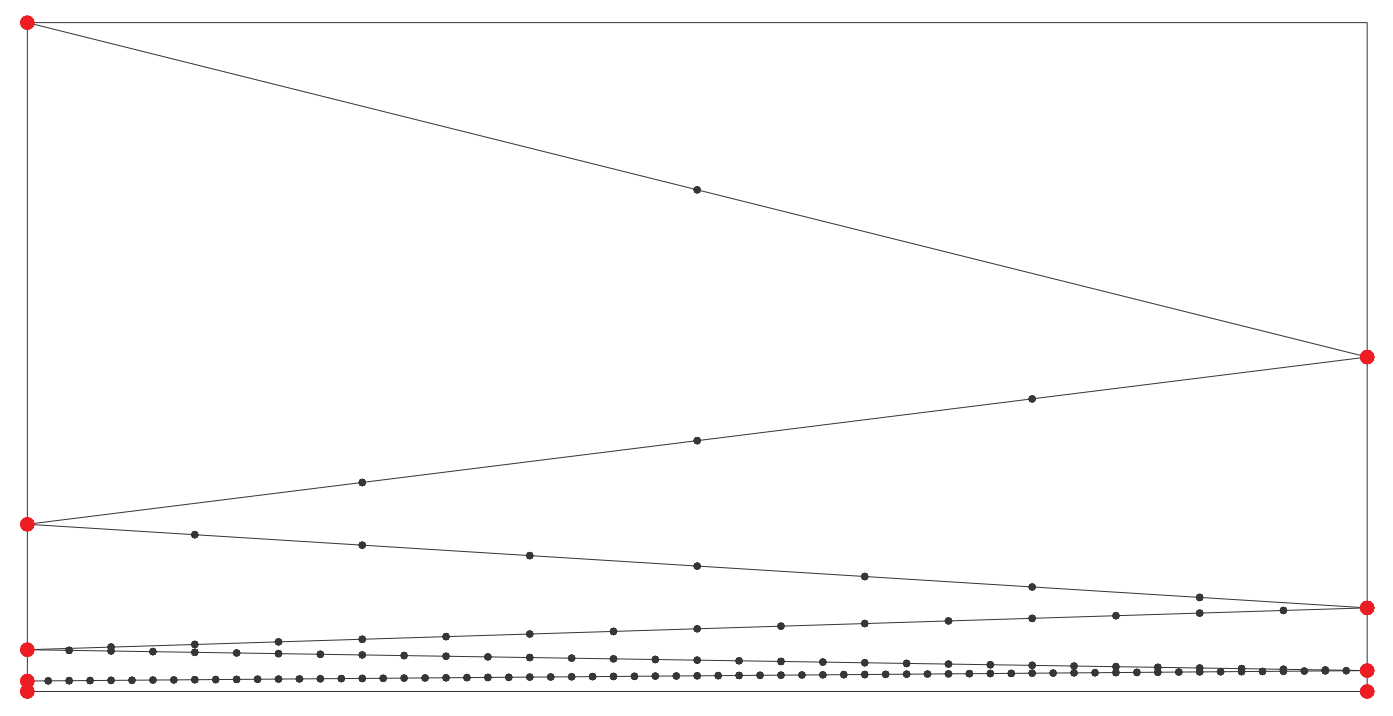

Fig. 2.1. Let the rectangle whose corners are the points of coordinates $(0,0),(1 / 2,0),(0,1 / 2)$ and $(1 / 2,1 / 2)$. In this figure we can see a representation of some elements of the sequence $\left\{x_{m}\right\}_{m \geqslant 2}$ of the Example 2.4. In particular, we can see a representation, on both sides of the rectangle, of some elements of the subsequences $\left\{x_{2^{2 n-1}}\right\}_{n}$ and $\left\{x_{2^{2 n}}\right\}_{n}$, respectively. This yields, in part, a visual intuition of the convergence of these subsequences to $(0,0)$ and $(1 / 2,0)$, respectively. 
On the other hand, given $p \in \mathbb{N}$ there exists $m_{p}$ such that if $m>m_{p}$ then:

In case i) we have:

i.1) $m+p$ is an element of the set $\left\{2^{2 n_{m}-1}+j+1, \ldots, 2^{2 n_{m}}-1,2^{2 n_{m}}\right\}$ or,

i.2) $m+p$ is an element of the set $\left\{2^{2 n_{m}}+1, \ldots, 2^{2 n_{m}+1}-2,2^{2 n_{m}+1}-1\right\}$.

In case ii) we have:

ii.1) $m+p$ is an element of the set $\left\{2^{2 n_{m}}+j+1, \ldots, 2^{2 n_{m}+1}-1,2^{2 n_{m}+1}\right\}$

or,

ii.2) $m+p$ is an element of the set $\left\{2^{2 n_{m}+1}+1, \ldots, 2^{2 n_{m}+2}-2,2^{2 n_{m}+2}-1\right\}$.

Next we show that $\left\{x_{m}\right\}_{m \geqslant 2}$ is G-Cauchy.

In case i.1) we have:

$$
\begin{aligned}
d\left(x_{m}, x_{m+p}\right)= & \frac{m+p-2^{2 n_{m}-1}}{2^{2 n_{m}}}-\frac{m-2^{2 n_{m}-1}}{2^{2 n_{m}}}+\frac{1}{2^{2 n_{m}-1}} \\
& -\frac{m-2^{2 n_{m}-1}}{2^{2 n_{m}} 2^{2 n_{m}-1}}-\frac{1}{2^{2 n_{m}-1}}+\frac{m+p-2^{2 n_{m}-1}}{2^{2 n_{m}} 2^{2 n_{m}-1}} \\
= & \frac{p}{2^{2 n_{m}}}+\frac{p}{2^{2 n_{m}} 2^{2 n_{m}-1}}<\frac{p}{2^{2 n_{m}}}+\frac{p}{2^{2 n_{m}}}=\frac{p}{2^{2 n_{m}-1}} .
\end{aligned}
$$

In case ii.1) we have:

$$
\begin{aligned}
d\left(x_{m}, x_{m+p}\right) & =\frac{p}{2^{2 n_{m}+1}}+\frac{p}{2^{2 n_{m}} 2^{2 n_{m}+1}} \\
& <\frac{p}{2^{2 n_{m}+1}}+\frac{p}{2^{2 n_{m}+1}}=\frac{p}{2^{2 n_{m}}} .
\end{aligned}
$$

In case i.2) we put: 
$p_{1}=2^{2 n_{m}}-m$,

$p_{2}=m+p-2^{2 n_{m}}, p_{1}+p_{2}=p$, and we shall use the following relation:

$$
d\left(x_{m}, x_{m+p}\right) \leqslant d\left(x_{m}, x_{2^{2 n_{m}}}\right)+d\left(x_{2^{2 n_{m}}}, x_{m+p}\right) .
$$

The computation of $d\left(x_{m}, x_{2^{2 n m}}\right)$ follows from case $\left.i .1\right)$ and we can put $d\left(x_{m}, x_{2^{2 n_{m}}}\right)=d\left(x_{m}, x_{m+p_{1}}\right)$, therefore:

$$
d\left(x_{m}, x_{m+p_{1}}\right)<\frac{p_{1}}{2^{2 n_{m}-1}} .
$$

The computation of $d\left(x_{2^{2 n m}}, x_{m+p}\right)$ follows from case ii.1), and thus:

$$
d\left(x_{2^{2 n_{m}}}, x_{m+p}\right)<\frac{p_{2}}{2^{2 n_{m}}}
$$

so:

$$
\begin{aligned}
d\left(x_{m}, x_{m+p}\right) & \leqslant d\left(x_{m}, x_{2^{2 n_{m}}}\right)+d\left(x_{2^{2 n_{m}}}, x_{m+p}\right) \\
& <\frac{p_{1}}{2^{2 n_{m}-1}}+\frac{p_{2}}{2^{2 n_{m}}} \\
& <\frac{p_{1}}{2^{2 n_{m}-1}}+\frac{p_{2}}{2^{2 n_{m}-1}}=\frac{p}{2^{2 n_{m}-1}} .
\end{aligned}
$$

In case ii.2) we put:

$$
\begin{aligned}
& p_{1}=2^{2 n_{m}+1}-m \\
& p_{2}=m+p-2^{2 n_{m}+1}, p_{1}+p_{2}=p \text {, and we shall use the following relation: } \\
& \qquad d\left(x_{m}, x_{m+p}\right) \leqslant d\left(x_{m}, x_{2^{2 n}+1}\right)+d\left(x_{2^{2 n}+1}, x_{m+p}\right) .
\end{aligned}
$$

The computation of $d\left(x_{m}, x_{2^{2 n_{m}+1}}\right)$ follows from case ii.1) and we can put $d\left(x_{m}, x_{2^{2 n_{m}+1}}\right)=d\left(x_{m}, x_{m+p_{1}}\right)$, therefore: 


$$
d\left(x_{m}, x_{m+p_{1}}\right)<\frac{p_{1}}{2^{2 n_{m}}} .
$$

The computation of $d\left(x_{2^{2 n m+1}}, x_{m+p}\right)$ follows from case $\left.i .1\right)$ and thus:

$$
d\left(x_{2^{2 n m+1}}, x_{m+p}\right)<\frac{p_{2}}{2^{2 n_{m}+1}}
$$

so:

$$
\begin{aligned}
d\left(x_{m}, x_{m+p}\right) & \leqslant d\left(x_{m}, x_{2^{2 n_{m}+1}}\right)+d\left(x_{2^{2 n_{m}+1}}, x_{m+p}\right) \\
& <\frac{p_{1}}{2^{2 n_{m}}}+\frac{p_{2}}{2^{2 n_{m}+1}} \\
& <\frac{p_{1}}{2^{2 n_{m}}}+\frac{p_{2}}{2^{2 n_{m}}}=\frac{p}{2^{2 n_{m}}}
\end{aligned}
$$

We deduce that $d\left(x_{m}, x_{m+p}\right)<\frac{p}{2^{2 n_{m}-1}}$, and for some $n_{m} \in \mathbb{N}$, for all $m>m_{p}$.

Therefore for each $t>0$ and $p \in \mathbb{N}$ :

$$
\lim _{m} M_{d}\left(x_{m+p}, x_{m}, t\right)=\lim _{m} \frac{t}{t+d\left(x_{m}, x_{m+p}\right)}=\lim _{m} \frac{t}{t+\frac{p}{2^{2 n_{m}-1}}}=1 .
$$

So, $\left\{x_{m}\right\}_{m \geqslant 2}$, is a non-convergent G-Cauchy sequence in the compact fuzzy metric space $\left(X, M_{d}, *\right)$. Thus the compact fuzzy metric space $\left(X, M_{d}, *\right)$ is not G-complete.

Example 2.5. Let $X=\left\{\left(x_{1}, x_{2}, x_{3}\right) \in \mathbb{R}^{3}: x_{1}^{2}+x_{2}^{2}=1\right.$ and $\left.0 \leqslant x_{3} \leqslant \frac{1}{2}\right\}$. Obviously $(X, d)$ is a compact metric space, where $d$ is the metric given by $d(x, y)=\left|y_{1}-x_{1}\right|+\left|y_{2}-x_{2}\right|+\left|y_{3}-x_{3}\right|$, with $x=\left(x_{1}, x_{2}, x_{3}\right), y=\left(y_{1}, y_{2}, y_{3}\right)$ and $z=\left(z_{1}, z_{2}, z_{3}\right)$. Hence $\left(X, M_{d}, *\right)$ is a compact fuzzy metric space.

Construct a sequence $\left\{x_{m}\right\}_{m \geqslant 2}$, in $\left(X, M_{d}, *\right)$ as follows:

Since for a given $m \geqslant 2$ there exists $n_{m}$ such that $2^{n_{m}} \leqslant m<2^{n_{m}+1}$, we define: 


$$
x_{m}=\left(\cos \left(\frac{2 \pi\left(m-2^{n_{m}}\right)}{2^{n_{m}}}\right), \sin \left(\frac{2 \pi\left(m-2^{n_{m}}\right)}{2^{n_{m}}}\right), \frac{1}{m}\right) .
$$

It is easy to check (see Figure 2.2) that $\left\{x_{2^{n}}\right\}_{n}$ and $\left\{x_{3.2^{n}}\right\}_{n}$ are subsequences of $\left\{x_{m}\right\}_{m \geqslant 2}$, where $x_{2^{n}}=\left(1,0, \frac{1}{2^{n}}\right)$ and $x_{3.2^{n}}=\left(-1,0, \frac{1}{3.2^{n}}\right)$, $n \in \mathbb{N}$. We have that $\lim _{n} x_{2^{n}}=(1,0,0)$ and $\lim _{n} x_{3.2^{n}}=(-1,0,0)$, and thus $\left\{x_{m}\right\}_{m \geqslant 2}$ is not convergent. 


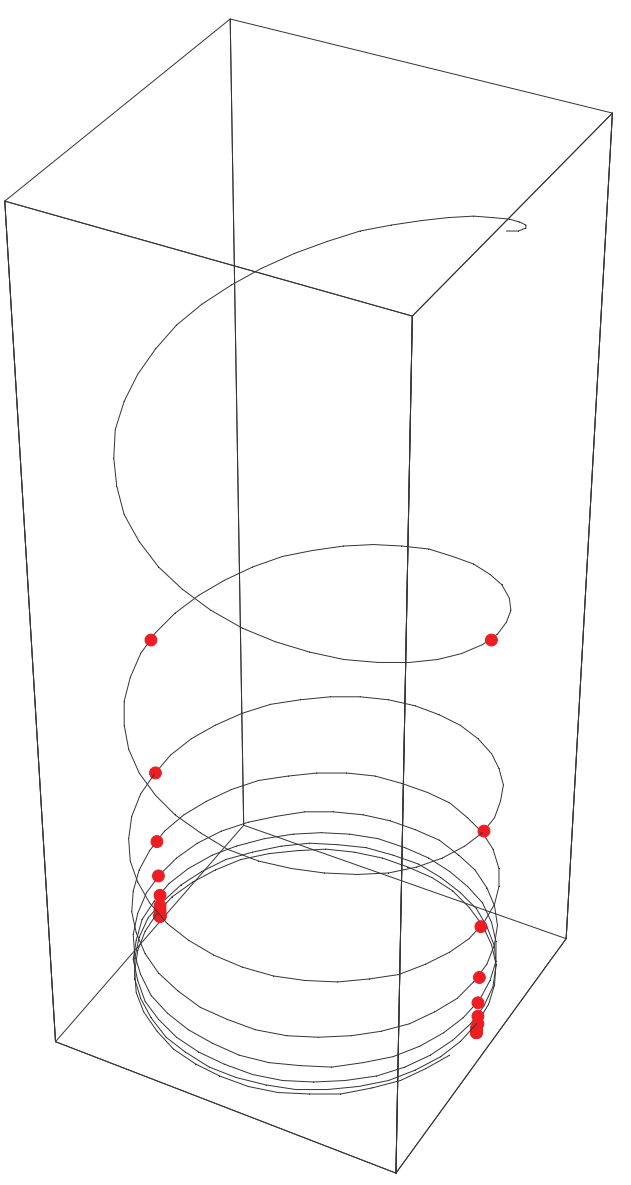

Fig. 2.2. Helix with radius 1 and decreasing step. 
Given $p \in \mathbb{N}$ there exists $m_{p}$ such that if $m>m_{p}$ then:

i) $2^{n_{m}} \leqslant m<2^{n_{m}+1}$ and $2^{n_{m}} \leqslant m+p<2^{n_{m}+1}$,

or

ii) $2^{n_{m}} \leqslant m<2^{n_{m}+1}$ and $2^{n_{m}+1} \leqslant m+p<2^{n_{m}+2}$

In case i) we have:

$$
\begin{aligned}
d\left(x_{m}, x_{m+p}\right)= & \left|\cos \left(\frac{2 \pi\left(m-2^{n_{m}}\right)}{2^{n_{m}}}\right)-\cos \left(\frac{2 \pi\left(m+p-2^{n_{m}}\right)}{2^{n_{m}}}\right)\right| \\
& +\left|\sin \left(\frac{2 \pi\left(m-2^{n_{m}}\right)}{2^{n_{m}}}\right)-\sin \left(\frac{2 \pi\left(m+p-2^{n_{m}}\right)}{2^{n_{m}}}\right)\right| \\
& +\left|\frac{1}{m}-\frac{1}{m+p}\right| \\
\leqslant & 4\left|\sin \left(\frac{2 \pi p}{2^{n_{m}+1}}\right)\right|+\frac{1}{m}-\frac{1}{m+p} .
\end{aligned}
$$

In case ii) we put:

$$
\begin{aligned}
& p_{1}=2^{n_{m}+1}-m \\
& p_{2}=m+p-2^{n_{m}+1}, p_{1}+p_{2}=p \\
& d\left(x_{m}, x_{m+p}\right) \leqslant d\left(x_{m}, x_{2^{n_{m}}}\right)+d\left(x_{2^{n_{m}}}, x_{m+p}\right) \\
& \leqslant 4\left|\sin \left(\frac{2 \pi p_{1}}{2^{n_{m}+1}}\right)\right|+4\left|\sin \left(\frac{2 \pi p_{2}}{2^{n_{m}+2}}\right)\right|+\frac{1}{m}-\frac{1}{m+p} .
\end{aligned}
$$

Therefore for each $t>0$ and $p \in \mathbb{N}$ :

$$
\lim _{m} M_{d}\left(x_{m+p}, x_{m}, t\right)=\lim _{m} \frac{t}{t+d\left(x_{m}, x_{m+p}\right)}=1 .
$$

So, $\left\{x_{m}\right\}_{m \geqslant 2}$, is a non-convergent $G$-Cauchy sequence in the compact fuzzy metric space $\left(X, M_{d}, *\right)$. Thus the compact fuzzy metric space $\left(X, M_{d}, *\right)$ is not G-complete. 


\section{Chapter 3}

\section{Complexity analysis}

\subsection{Introduction}

The complexity (quasi-metric) space has been introduced as a part of the development of a topological foundation for the complexity analysis of algorithms (see [50]). Applications of this theory to the complexity analysis of Divide and Conquer algorithms has been discussed by Schellekens in [50].

In Section 3.2 we recall the notion of complexity space and in Section 3.3 we recall the recurrence equation associated to Divide and Conquer algorithms. In Section 3.4 we introduce a new complexity quasi-metric space whose complexity functions are defined on $\mathbb{N} \times \mathbb{N}$, and show that it provides a suitable framework for the complexity analysis of the expoDC algorithm (see [6]) whose complexity depends on two parameters. In fact, the model provided by Schellekens's construction does not provide a suitable framework to analyze those algorithms whose execution time depends on more than one parameter.

The main results of Section 3.4 are contained in [44].

In the rest of Section 3.4 we recall some well-known concepts and ideas from the classical theory of complexity (see [42], [43], [50] and [51]). 
Given a partial recursive function $f$, and a programming language $\mathcal{L}$, let $[f]$ be the set of all programs of $\mathcal{L}$ computing a partial recursive function which approximates $f$ (in the usual pointwise ordering on partial functions). We will use $P, Q, \ldots$ in what follows to denote programs.

A complexity (function) measure is a binary partial function $C(k, n)$ on $\mathbb{N}^{2}$ satisfying the Blum axioms:

1) $C(k, n)$ is defined if and only if the program with coding $k$ converges on input $n$.

2) the predicate $C(k, n) \leq y$ is recursive.

So $C(k, n)$ represents the complexity of a program $P$ (with code $k$ ) on input $n$.

We denote the complexity of a given program $P$ for a given complexity measure $C$ by $C_{P}$. We assume that for any given program and complexity measure $C, C_{P}$ is non-zero on all inputs and in case $C_{P}$ is not defined on input $n$, we let $C_{P}(n)=\infty$. This last convention is standard in the theory of abstract complexity measures.

We reserve the symbol $T$ to indicate the function on the natural numbers with constant value $\infty$. A program which is undefined on every input thus has $T$ as complexity function. The requirement that complexity measures take non zero values is made to eliminate division by zero in the definition of the complexity distance. The requirement is convenient but not essential. In fact, for the case of asymptotic complexity analysis, the condition can for instance be guaranteed by requiring that complexity measures count the reading of an input as one step, when this is not already the case. This shift of the complexity measure by one unit obviously will not affect the result of the asymptotic analysis. 
In practice complexity measures typically count "steps" (increments, comparisons, space allocations, etc) made during the calculation of an output value. So by requirement that complexity measures take non zero values, the complexity functions are guaranteed to be bounded from below, that is for all $k$ there exists $c>0$ such that for all $n$ we have $C(k, n) \geq c$. Typically the constant $c$ will be a unit value used to measure steps carried out by the program. Even if one allows different steps to be measured by different constant values, since only finitely many different kinds of steps will occur in the code of a given program, the condition will not be violated. Neither will the average-case, worst-case or asymptotic approach to complexity violate the condition, as one can easily verify.

Let $\mathcal{C}$ be the set of all functions from $(0, \infty]^{w}$ which are bounded from below. This set includes all complexity function which can occur in practice, but of course not every element of the space corresponds to a complexity function of a program.

The set contains functions which take values in the reals, rather than the natural numbers, in order to include complexities obtained by average-case or asymptotic analysis.

As mentioned above, the value $\infty$ for a complexity function of a program corresponds to an undefined output value for this program, which motivates the inclusión of infinity as a possible function value.

Following the usual terminology of "asymptotic time", for each $g \in \mathcal{C}$ we define:

$$
\mathcal{O}(g):=\left\{f \in \mathcal{C}: \text { there exists } c>0 \text { and } n_{0} \in w \text { such that } f(n) \leq c g(n)\right.
$$

for all $\left.n \geq n_{0}\right\}$. 


\subsection{The complexity space}

The theory of complexity (quasi-metric) spaces, introduced by M. Schellekens in [50], constitutes a part of the research in Theoretical Computer Science and Topology and provides a mathematical model for the complexity analysis of algorithms. Applications of fixed point methods on complexity spaces to the complexity analysis of Divide and Conquer algorithms are also presented in $[50]$.

Further contributions to the development of the theory of complexity spaces and other related structures, and its application to Quicksort algorithm may be found in [18], [38], [42], [43] and [40], and in [39] and [51] respectively.

Definition 3.1 [50]. The complexity (quasi-metric) space is the pair $\left(\mathcal{C}, d_{\mathcal{C}}\right)$, where

$$
\mathcal{C}=\left\{f: \omega \rightarrow(0, \infty]: \sum_{n=0}^{\infty} 2^{-n} \frac{1}{f(n)}<\infty\right\},
$$

and $d_{\mathcal{C}}$ is the quasi-metric on $\mathcal{C}$ given by

$$
d_{\mathcal{C}}(f, g)=\sum_{n=0}^{\infty} 2^{-n}\left(\left(\frac{1}{g(n)}-\frac{1}{f(n)}\right) \vee 0\right),
$$

for all $f, g \in \mathcal{C}$. (We adopt the convention that $1 / \infty=0$.) The elements of $\mathcal{C}$ are called complexity functions.

According to [50, Section 4] the intuition behind the complexity distance $d_{\mathcal{C}}(f, g)$ measures relative progress made in lowering the complexity by replacing the complexity function $f$ by the complexity function $g$. Thus $d_{\mathcal{C}}(f, g)=0$ if and only if $f(n) \leq g(n)$ for all $n \in \omega$; and, hence, condition $d_{\mathcal{C}}(f, g)=0$, with $f \neq g$, can be interpreted as $f$ is more efficient than $g$ on all inputs. Observe that the above information is not provided by using the metric $\left(d_{\mathcal{C}}\right)^{s}$ 
because from the value $\left(d_{\mathcal{C}}\right)^{s}(f, g)$ is not possible to determine which complexity function would be more efficient.

Let us recall that a quasi-metric $d$ on a set $X$ induces a partial order $\leq_{d}$ on $X$ given by

$$
x \leq_{d} y \Leftrightarrow d(x, y)=0
$$

If we denote by $T$ the complexity function on $\omega$ with constant value $\infty$, then it is clear that $T$ is the maximum of $\mathcal{C}$ for the partial order $\leq_{d_{C}}$.

\subsection{Recursive algorithms}

Computer scientists sometimes classify algorithms according to the design strategies they employ. Three of the most commonly used types of algorithms are called Greedy algorithms, Divide \& Conquer algorithms, and Backtracking algorithms.

Recall that in Divide \& Conquer algorithms, we divide a large problem into smaller subproblems. We then solve each subproblem separately and combine the results into a solution to the whole problem. The procedure is particularly attractive when each subproblem is of the same type as the original so that it can be solved by reapplying the technique, until finally sub-subproblems are reached which are trivially simple to solve. This particular approach to Divide \& Conquer algorithms is called recursion. In other words a Divide \& Conquer algorithm solve a problem by recursively splitting it into subproblems each of which is solved separately by the same algorithm, after which the results are combined into a solution of the original problem. 
The complexity $C$ of a Divide \& Conquer algorithm typically is the solution to a recurrence equation $\varepsilon$ of the form $C(1)=c$ and $C(n)=a C\left(\frac{n}{b}\right)+h(n)$ for all $n>1$, where $a>1$ represents the number of subproblems a problem is divided into, $\frac{n}{b}$ represents the size of each subproblem and $h(n)$ represents the complexity required to combine the subproblems of a problem of size $n$ into the solution, (see, for instance [6], [50] and [51]).

A Divide \& Conquer algorithm with a recurrence equation $\varepsilon$ of the kind defined above, induces a functional $\Phi_{\varepsilon}$ on the complexity space $(\mathcal{C}, d)$ defined by $\Phi_{\varepsilon}=\lambda f \lambda n$. If $n=1$ then $c$ else $a f\left(\frac{n}{b}\right)+h(n)$, where $n$ ranges over powers of the form $b^{k}$ for $k \geq 0$. Such functionals are used in carrying out the complexity analysis of programs and map any function of the complexity space to a function defined via pointwise addition and scalar multiplication, where the operations correspond to operations carried out by the Divide \& Conquer algorithm on the datastructures. In general any recursive algorithm induces a functional on the complexity space $(\mathcal{C}, d)$.

\subsection{Contraction maps on complexity spaces and expoDC algorithms}

ExpoDC algorithms (see [6]) compute the exponentiation $x=a^{n}$, with $a$ and $n$ two integers by using "Divide and Conquer" techniques. For simplicity, we will assume that $n>0$.

Next we discuss the complexity analysis of expoDC algorithms by using techniques of Denotational Semantics. This is done by showing that the recurrence inequation associated to an expoDC algorithm gives rise to a contraction map on a suitable quasi-metric space which is constructed here and 
whose elements are "complexity" functions of the function space $[0, \infty)^{\mathbb{N} \times \mathbb{N}}$. We prove that this contraction map has a unique fixed point, which is the maximal element, with respect to the partial order induced by the quasimetric, of the set of solutions of the recurrence inequation. The complexity of such an algorithm is represented via this maximal element.

Recall that by a contraction map on a quasi-metric space $(X, d)$ we mean a self-map $f$ on $X$ such that $d(f x, f y) \leq k d(x, y)$ for all $x, y \in X$, where $k$ is a constant with $0 \leq k<1$. The number $k$ is called a contraction constant for $f$.

It is clear that if $f$ is a contraction map on a quasi-metric space $(X, d)$ with contraction constant $k$, then $f$ is a contraction map on the metric space $\left(X, d^{s}\right)$ with contraction constant $k$.

We formulate the following detailed form of the Banach contraction principle, which will be useful later on.

Theorem 3.1. Let $f$ be a contraction map on a complete metric space $(X, d)$. Then, for any $x \in X$, the sequence of iterations $\left(f^{n} x\right)_{n \in \omega}$ is convergent in $(X, d)$ to a point $x_{0}$ which is the unique fixed point of $f$.

The following useful result is an immediate consequence of [42, Theorem 1 and Remark on p. 317].

Theorem 3.2. The quasi-metric space $\left(\mathcal{C}, d_{\mathcal{C}}\right)$ is bicomplete.

Since complexity functions are defined on $\omega$, the quasi-metric space $\left(\mathcal{C}, d_{\mathcal{C}}\right)$ does not provide a suitable framework to analyze an algorithm for which the execution time depends on more than one parameter. Here we introduce a new complexity quasi-metric space whose complexity functions are defined 
on $\mathbb{N} \times \mathbb{N}$, and show that it provides a suitable framework for the complexity analysis of the expoDC algorithm which takes two parameters $a$ and $n$ as inputs and computes the $n$-th power of the value $a$ (see for instance [6, Section 7.7] for a detailed discussion on this kind of algorithm). In particular, we show that the recurrence inequation associated to an expoDC algorithm gives rise to a contraction map on the new complexity space. The complexity of such an algorithm is represented via the fixed point of this contraction map, which is the maximal element, with respect to the partial order induced by the quasi-metric, of the set of solutions of the recurrence inequation. This approach provides an application of typical Denotational Semantics techniques to the context of Complexity Theory.

In order to obtain, in this context, an appropriate mathematical model to analyze those algorithms whose execution time depends on two parameters it seems natural to consider complexity functions belonging to the function space $(0, \infty]^{\omega \times \omega}$, and then construct a suitable modification of the complexity quasi-metric $d_{\mathcal{C}}$ that preserves its nice properties. A detailed study of this general approach will be discussed elsewhere. However, for our purposes here it suffices to consider the following subset of $[0, \infty)^{\mathbb{N} \times \mathbb{N}}$ :

$$
\mathcal{C}_{0, c}:=\{f: \mathbb{N} \times \mathbb{N} \rightarrow[0, \infty): f(m, 1)=0, \text { and } f(m, n) \geq c \text { for } n>1\}
$$

where $c$ is a positive real number.

Now, for each $f \in \mathcal{C}_{0, c}$ and each $m \in \mathbb{N}$, define the function $f(m): \omega \rightarrow$ $(0, \infty]$ by

$$
f(m)(0)=f(m)(1)=\infty, \quad \text { and } \quad f(m)(n)=f(m, n) \text { for } n>1 .
$$

Then it is clear that $f(m) \in \mathcal{C}$.

Next we define a function $d_{\mathcal{C}_{0, c}}: \mathcal{C}_{0, c} \times \mathcal{C}_{0, c} \rightarrow[0, \infty)$ by 


$$
d_{\mathcal{C}_{0, c}}(f, g)=\sum_{m=1}^{\infty} 2^{-m}\left[\sum_{n=2}^{\infty} 2^{-n}\left(\left(\frac{1}{g(m, n)}-\frac{1}{f(m, n)}\right) \vee 0\right)\right],
$$

for all $f, g \in \mathcal{C}_{0, c}$.

Note that, indeed, $d_{\mathcal{C}_{0, c}}$ is well-defined because $d_{\mathcal{C}_{0, c}}(f, g) \leq 1 / 2 c$, for all $f, g \in \mathcal{C}_{0, c}$.

Note also that

$$
d_{\mathcal{C}_{0, c}}(f, g)=\sum_{m=1}^{\infty} 2^{-m} d_{\mathcal{C}}(f(m), g(m))
$$

for all $f, g \in \mathcal{C}_{0, c}$.

Theorem 3.3 below will be crucial in the following.

Proposition 3.1. For each $m \in \mathbb{N}$, the set

$$
\mathcal{C}_{m}:=\left\{f(m): f \in \mathcal{C}_{0, c}\right\}
$$

is closed in the metric space $\left(\mathcal{C},\left(d_{\mathcal{C}}\right)^{s}\right)$.

Proof. Fix $m_{0} \in \mathbb{N}$. Let $f \in \mathcal{C}$ and let $\left(f_{k}\right)_{k \in \mathbb{N}}$ be a sequence in $\mathcal{C}_{0, c}$ such that $\left(d_{\mathcal{C}}\right)^{s}\left(f, f_{k}\left(m_{0}\right)\right) \rightarrow 0$ whenever $k \rightarrow \infty$. Then $f(0)=f(1)=\infty$ and $f(n) \geq c$ for all $n>1$. Define $h: \mathbb{N} \times \mathbb{N} \rightarrow[0, \infty)$ by $h(m, 1)=0$ and $h(m, n)=f(n)$ for all $n>1, m \in \mathbb{N}$. Clearly $h \in \mathcal{C}_{0, c}$. Since $f(n)=h\left(m_{0}\right)(n)$ for all $n>1$, it follows that $f \in \mathcal{C}_{m_{0}}$. We conclude that $\mathcal{C}_{m_{0}}$ is closed in $\left(\mathcal{C},\left(d_{\mathcal{C}}\right)^{s}\right)$.

Corollary 3.1. For each $m \in \mathbb{N}$, the quasi-metric space $\left(\mathcal{C}_{m},\left.d_{\mathcal{C}}\right|_{\mathcal{C}_{m}}\right)$ is bicomplete. 
Proof. By Theorem 3.2, $\left(\mathcal{C},\left(d_{\mathcal{C}}\right)^{s}\right)$ is a complete metric space. Since, by Proposition $3.1, \mathcal{C}_{m_{0}}$ is closed in $\left(\mathcal{C},\left(d_{\mathcal{C}}\right)^{s}\right)$, the result follows from [14, Theorem 4.3.11].

Theorem 3.3. The quasi-metric space $\left(\mathcal{C}_{0, c}, d_{\mathcal{C}_{0, c}}\right)$ is bicomplete.

Proof. For each $m \in \mathbb{N}$, the metric space $\left(\mathcal{C}_{m},\left(\left.d_{\mathcal{C}}\right|_{\mathcal{C}_{m}}\right)^{s}\right)$ is bounded. Furthermore, it is complete by Corollary 3.1 . Hence, the metric space $\left(\mathcal{C}_{0, c},\left(d_{\mathcal{C}_{0, c}}\right)^{s}\right)$ is complete by [14, Theorem 4.3.12].

We shall prove that the recurrence inequation associated to an expoDC algorithm gives rise to a contraction map on $\left(\mathcal{C}_{0, c}, d_{\mathcal{C}_{0, c}}\right)$. Then, by the Banach contraction principle, the contraction map has a unique fixed point, and then the complexity of the algorithm is represented via this fixed point because it is the maximal element with respect to the partial order $\leq_{d_{\mathcal{C}_{0, c}}}$, of the set of solutions of the recurrence inequation.

As we indicated above, the analysis of expoDC algorithm is for instance discussed in [6, Section 7.7], where the following recurrence inequation for this algorithm is obtained:

$$
T(m, n) \leq\left\{\begin{array}{l}
0, \quad \text { if } n=1, \\
T(m, n / 2)+M(m n / 2, m n / 2), \quad \text { if } n \text { is even } \\
T(m, n-1)+M(m,(n-1) m), \quad \text { otherwise }
\end{array}\right.
$$

for all $(m, n) \in \mathbb{N} \times \mathbb{N}$.

According to [6, Section 7.7], $M(m, n)$ denotes the time needed to multiply two integers of sizes $m$ and $n$, and $T(m, n)$ denotes the time spent multiplying when computing $a^{n}$, where $m$ is the size of $a$.

Moreover, we can assume that $M$ is monotone increasing, i.e., $M\left(m_{2}, n_{2}\right) \geq$ $M\left(m_{1}, n_{1}\right)$ whenever $m_{2} \geq m_{1}$ and $n_{2} \geq n_{1}$. By using the classical multiplication algorithm it then follows that $M(m, n) \in \mathcal{O}(m n)([6$, Section 7.7]). 
Let $M(1,1)=c>0$. Then, the recurrence (1) induces, in a natural way, the functional $\Phi$ defined on $\mathcal{C}_{0, c}$ by

$$
\Phi f(m, n)=\left\{\begin{array}{l}
0, \quad \text { if } n=1, \\
f(m, n / 2)+M(m n / 2, m n / 2), \quad \text { if } n \text { is even } \\
f(m, n-1)+M(m,(n-1) m), \quad \text { otherwise }
\end{array}\right.
$$

Observe that $\Phi$ is a self-map on $\mathcal{C}_{0, c}$, because for any $f \in \mathcal{C}_{0, c}$, we have $\Phi f(m, 1)=0$, and for $n>1, \Phi f(m, n) \geq M(1,1)=c$.

Note also that $\Phi$ is monotone increasing, i.e. $\Phi f \leq \Phi g$ whenever $f \leq g$.

Next we shall show that $\Phi$ is a contraction map on $\left(\mathcal{C}_{0, c}, d_{\mathcal{C}_{0, c}}\right)$ with contraction constant $3 / 4$.

Indeed, let $f \in \mathcal{C}_{0, c}$. Then $\Phi f(m, 1)=0$, and for $n>1, \Phi f(m, n) \geq$ $M(1,1)=c$. So $\Phi f \in \mathcal{C}_{0, c}$.

Now, given $f, g \in \mathcal{C}_{0, c}$, we have:

$$
d_{\mathcal{C}_{0, c}}(\Phi f, \Phi g)=\sum_{m=1}^{\infty} 2^{-m} d_{\mathcal{C}}(\Phi f(m), \Phi g(m)) .
$$

Choose an arbitrary $m \in \mathbb{N}$. Then

$$
d_{\mathcal{C}}(\Phi f(m), \Phi g(m))=\sum_{n=2}^{\infty} 2^{-n}\left(\left(\frac{1}{\Phi g(m, n)}-\frac{1}{\Phi f(m, n)}\right) \vee 0\right)
$$

Put

$$
a_{n}=2^{-n}\left(\left(\frac{1}{\Phi g(m, n)}-\frac{1}{\Phi f(m, n)}\right) \vee 0\right),
$$

for all $n \geq 2$. Observe that, in particular, $a_{2}=0$. Hence

$$
d_{\mathcal{C}}(\Phi f(m), \Phi g(m))=\sum_{n=3}^{\infty} a_{n}
$$


So, by reordering the serie $\sum_{n=3}^{\infty} a_{n}$, we obtain that

$$
d_{\mathcal{C}}(\Phi f(m), \Phi g(m))=\sum_{n=1}^{\infty}\left(a_{2 n+1}+a_{4 n}+a_{2(2 n+1)}\right)
$$

Therefore

$$
\begin{aligned}
a_{2 n+1} & =2^{-(2 n+1)}\left(\left(\frac{1}{\Phi g(m, 2 n+1)}-\frac{1}{\Phi f(m, 2 n+1)}\right) \vee 0\right) \\
& =2^{-(2 n+1)}\left(\left(\frac{1}{g(m, 2 n)+M(m, 2 m n)}-\frac{1}{f(m, 2 n)+M(m, 2 m n)}\right) \vee 0\right) \\
& =2^{-(2 n+1)}\left(\left(\frac{f(m, 2 n)-g(m, 2 n)}{(g(m, 2 n)+M(m, 2 m n)) f(m, 2 n)+M(m, 2 m n)}\right) \vee 0\right) \\
& \leq 2^{-(2 n+1)}\left(\left(\frac{f(m, 2 n)-g(m, 2 n)}{g(m, 2 n) f(m, 2 n)}\right) \vee 0\right) \\
& =2^{-(2 n+1)}\left(\left(\frac{1}{g(m, 2 n)}-\frac{1}{f(m, 2 n)}\right) \vee 0\right) .
\end{aligned}
$$

Similarly

$$
\begin{aligned}
a_{4 n} & =2^{-4 n}\left(\left(\frac{1}{\Phi g(m, 4 n)}-\frac{1}{\Phi f(m, 4 n)}\right) \vee 0\right) \\
& =2^{-4 n}\left(\left(\frac{1}{g(m, 2 n)+M(2 m n, 2 m n)}-\frac{1}{f(m, 2 n)+M(2 m n, 2 m n)}\right) \vee 0\right) \\
& \leq 2^{-4 n}\left(\left(\frac{1}{g(m, 2 n)}-\frac{1}{f(m, 2 n)}\right) \vee 0\right),
\end{aligned}
$$

and, also, 


$$
\begin{aligned}
a_{2(2 n+1)} & =2^{-2(2 n+1)}\left(\left(\frac{1}{\Phi g(m, 2(2 n+1))}-\frac{1}{\Phi f(m, 2(2 n+1))}\right) \vee 0\right) \\
& \leq 2^{-2(2 n+1)}\left(\left(\frac{1}{g(m, 2 n+1)}-\frac{1}{f(m, 2 n+1)}\right) \vee 0\right) .
\end{aligned}
$$

Putting, for each $n \geq 2$,

$$
b_{n}=2^{-n}\left(\left(\frac{1}{g(m, n)}-\frac{1}{f(m, n)}\right) \vee 0\right) \text {, }
$$

we deduce that

$$
\begin{aligned}
d_{\mathcal{C}}(\Phi f(m), \Phi g(m)) & \leq \sum_{n=1}^{\infty}\left(\left(2^{-1}+2^{-2 n}\right) b_{2 n}+2^{-(2 n+1)} b_{2 n+1}\right) \\
& \leq \frac{3}{4} \sum_{n=1}^{\infty}\left(b_{2 n}+b_{2 n+1}\right) \\
& =\frac{3}{4} \sum_{n=2}^{\infty} 2^{-n}\left(\left(\frac{1}{g(m, n)}-\frac{1}{f(m, n)}\right) \vee 0\right) \\
& =\frac{3}{4} d_{\mathcal{C}}(f(m), g(m)) .
\end{aligned}
$$

Consequently

$$
d_{\mathcal{C}_{0, c}}(\Phi f, \Phi g) \leq \frac{3}{4} \sum_{m=1}^{\infty} 2^{-m} d_{\mathcal{C}}(f(m), g(m))=\frac{3}{4} d_{\mathcal{C}_{0, c}}(f, g),
$$

and hence

$$
\left(d_{\mathcal{C}_{0, c}}\right)^{s}(\Phi f, \Phi g) \leq \frac{3}{4}\left(d_{\mathcal{C}_{0, c}}\right)^{s}(f, g)
$$

for all $f, g \in \mathcal{C}_{0, c}$.

Since, by Theorem $3.3,\left(\mathcal{C}_{0, c},\left(d_{\mathcal{C}_{0, c}}\right)^{s}\right)$ is a complete metric space, it follows from Theorem 3.1 that there exists a unique $f_{0} \in \mathcal{C}_{0, c}$ such that $\Phi f_{0}=f_{0}$. Therefore $f_{0}$ is a solution for the recurrence inequation (1). 
Next we shall deduce from our methods the known fact that $f_{0} \in \mathcal{O}\left(m^{2} n^{2}\right)$. Indeed, since $M(m, n) \in \mathcal{O}(m n)$ and $M(1,1)=c$, it follows that there exist $K \geq c$ and $n_{0}>1$ such that $M(m, n) \leq K m n$ for all $m, n \geq n_{0}$. Now define a function $h \in \mathcal{C}_{0, c}$ by $h(m, 1)=0$, and $h(m, n)=K n_{0}^{2} m^{2} n^{2}$ whenever $n>1$. An easy computation, taking into account that $M$ is monotone increasing, shows that $\Phi h \leq h$. Since $\Phi$ is also monotone increasing we obtain that $\Phi^{k} h \leq h$, for all $k \in \mathbb{N}$, and thus $d_{\mathcal{C}_{0, c}}\left(\Phi^{k} h, h\right)=0$, for all $k \in \mathbb{N}$. On the other hand, it follows from the Banach contraction principle that $\left(d_{\mathcal{C}_{0, c}}\right)^{s}\left(f_{0}, \Phi^{k} h\right) \rightarrow 0$ whenever $k \rightarrow \infty$; so, by the triangle inequality, $d_{\mathcal{C}_{0, c}}\left(f_{0}, h\right)=0$; and thus $f_{0} \leq h$. We conclude that $f_{0} \in \mathcal{O}\left(m^{2} n^{2}\right)$.

Finally, we shall prove that $f_{0}$ is the maximal element of the set of solutions of (1), with respect to the partial order $\leq_{d_{\mathcal{C}_{0, c}}}$.

To this end, let $g \in \mathcal{C}_{0, c}$ satisfying the recurrence inequation (1). Then $g \leq \Phi g$, and, hence, $d_{\mathcal{C}_{0, c}}(g, \Phi g)=0$. Therefore

$$
\begin{aligned}
d_{\mathcal{C}_{0}, c}\left(g, f_{0}\right) & \leq d_{\mathcal{C}_{0, c}}(g, \Phi g)+d_{\mathcal{C}_{0}, c}\left(\Phi g, \Phi f_{0}\right)+d_{\mathcal{C}_{0}, c}\left(\Phi f_{0}, f_{0}\right) \\
& =d_{\mathcal{C}_{0, c}}\left(\Phi g, \Phi f_{0}\right) \\
& \leq \frac{3}{4} d_{\mathcal{C}_{0, c}}\left(g, f_{0}\right)
\end{aligned}
$$

Consequently $d_{\mathcal{C}_{0, c}}\left(g, f_{0}\right)=0$, and thus $g \leq_{d_{\mathcal{C}_{0, c}}} f_{0}$, or, equivalently, $g \leq$ $f_{0}$.

We conclude that $f_{0}$ is the maximal element of the set of solutions of (1), with respect to the partial order $\leq_{d_{\mathcal{C}_{0, c}}}$.

Remark 3.1. Note that the requirement that $f(m, n) \geq c$ for all $n>1$, in 
3.4. Contraction maps And expoDC ALgorithms

the definition of $\mathcal{C}_{0, c}$ is justified by the fact that

$$
T(m, n) \geq \sum_{j=1}^{n-1} M(m, j m-j+1),
$$

for all $(m, n) \in \mathbb{N} \times \mathbb{N}$ (see [6, p. 244]), and hence

$$
T(m, n) \geq M(m, m) \geq M(1,1)=c .
$$




\section{Chapter 4}

\section{Application of fuzzy quasi-metrics to the theory of asymptotic complexity of algorithms}

\subsection{Introduction}

In [50], M. Schellekens began the development of complexity (quasi-metric) space (Chapter 3). The intuition behind the "complexity distance" between complexity functions $f$ and $g$, is that $d(f, g)$ measures relative progress in lowering the complexity by replacing $f$ by $g$, therefore this model gives a suitable computational interpretation of the fact that a program $P$ is more efficient than other program $Q$ on all the inputs. However this model does not provide a suitable framework to measure the relative progress between complexity functions $f$ and $g$ in the case of $f$ is "only" asymptotically more efficient than $g$.

We introduce and study in this chapter a fuzzy quasi-metric on the set 
of complexity functions, which provides a satisfactory measurement of the distance from the complexity function $f$ to the complexity function $g$ in the case of $f$ is asymptotically more efficient than $g$. We also obtain a version of the Banach fixed point theorem in this context which is applied to the asymptotic complexity analysis of Divide \& Conquer algorithms an Quicksort algorithms, respectively.

The main results of this chapter may be found in [46].

Let us recall (Section 3.2) that given the complexity quasi-metric space $\left(\mathcal{C}, d_{\mathcal{C}}\right)$, then the condition $d_{\mathcal{C}}(f, g)=0$ is equivalent to the fact that $f(n) \leq$ $g(n)$ for all $n \in w$. Hence, if the measure of complexity is the running time of computing, and $f$ and $g$, with $f \neq g$, represent the running time of two different algoritms, then $d_{\mathcal{C}}(f, g)=0$ can be interpreted as "the efficiency of $P$ is better than $Q$ on all inputs" or simply, $f$ is "more efficient" than $g$ on all inputs.

We say that $f \in \mathcal{C}$ is asymptotically more efficient than $g \in \mathcal{C}$ if $f \in \mathcal{O}(g)$ with $f \neq g$ and $c \leq 1$.

Therefore, it appears in a natural way the interesting problem of construction a kind of quasi-metric which provides a satisfactory measurement of the distance from $f$ to $g$ in the case that $f$ is asymptotically more efficient than $g$.

The following example shows that $d_{\mathcal{C}}$ is not sensitive to describe this situation in general.

Example 4.1. Consider de functions $f, g, h \in \mathcal{C}$ given by $f(n)=n+2$, $g(n)=2^{n} /\left(n^{2}+1\right)$ and $h(n)=n+1$ for all $n \in w$. An easy computation shows that $f(n)>g(n)$ for $n=0,1, \ldots, 10$, and $f(n)<g(n)$ for $n \geq 11$. 
Hence $f$ is asymptotically more efficient than $g$. Moreover, we have that $h(n)<f(n)$ for all $n \in w$. However $d_{\mathcal{C}}(f, g)>5 / 6>d_{\mathcal{C}}(f, h)$.

\subsection{The complexity fuzzy quasi-metric space}

In order to define the complexity fuzzy quasi-metric space we construct the following auxiliary function.

For each $f, g \in \mathcal{C}$ and $t>0$ let:

$$
D_{\mathcal{C}}(f, g, t)=\sum_{k=n}^{\infty} 2^{-k}\left(\left(\frac{1}{g(k)}-\frac{1}{f(k)}\right) \vee 0\right)
$$

where $t \in(n, n+1], n \in \omega$.

Remark 4.1. Note that, for each $f, g \in \mathcal{C}$ and $t>0$, we have:

$$
D_{\mathcal{C}}(f, g, t) \leqslant \sum_{k=0}^{\infty} 2^{-k}\left(\left(\frac{1}{g(k)}-\frac{1}{f(k)}\right) \vee 0\right)=d_{\mathcal{C}}(f, g)
$$

In particular, for each $f, g \in \mathcal{C}$ and $t \in(0,1]$, we obtain $D_{\mathcal{C}}(f, g, t)=$ $d_{\mathcal{C}}(f, g)$.

Lemma 4.1. For each $f, g, h \in \mathcal{C}$ and $t, s>0$, it follows:

$$
D_{\mathcal{C}}(f, g, t+s) \leqslant D_{\mathcal{C}}(f, h, t)+D_{\mathcal{C}}(h, g, s)
$$

Proof. Let $t \in(n, n+1]$ and $s \in(m, m+1]$, with $n, m \in w$. Then $t+s \in(n+m, n+m+1]$ or $t+s \in(n+m+1, n+m+2]$. Consequently: 


$$
\begin{aligned}
D_{\mathcal{C}}(f, g, t+s) & \leqslant \sum_{k=n+m}^{\infty} 2^{-k}\left(\left(\frac{1}{g(k)}-\frac{1}{f(k)}\right) \vee 0\right) \\
& \leqslant \sum_{k=n+m}^{\infty} 2^{-k}\left(\left(\frac{1}{h(k)}-\frac{1}{f(k)}\right) \vee 0\right)+\sum_{k=n+m}^{\infty} 2^{-k}\left(\left(\frac{1}{g(k)}-\frac{1}{h(k)}\right) \vee 0\right) \\
& \leqslant \sum_{k=n}^{\infty} 2^{-k}\left(\left(\frac{1}{h(k)}-\frac{1}{f(k)}\right) \vee 0\right)+\sum_{k=m}^{\infty} 2^{-k}\left(\left(\frac{1}{g(k)}-\frac{1}{h(k)}\right) \vee 0\right) \\
& =D_{\mathcal{C}}(f, h, t)+D_{\mathcal{C}}(h, g, s) .
\end{aligned}
$$

The proof is finished

Theorem 4.1. For each $(f, g, t) \in \mathcal{C} \times \mathcal{C} \times[0, \infty)$ let

$$
\begin{aligned}
M_{\mathcal{C}}(f, g, 0) & =0, \\
M_{\mathcal{C}}(f, g, t) & =\frac{t}{t+D_{\mathcal{C}}(f, g, t)},
\end{aligned}
$$

whenever $t>0$. Then $\left(M_{\mathcal{C}}, \wedge\right)$ is a fuzzy quasi-metric on $\mathcal{C}$.

Furthermore for each $f, g \in \mathcal{C}, M_{\mathcal{C}}(f, g, t)=M_{d_{\mathcal{C}}}(f, g, t)$ whenever $t \in$ $(0,1]$, and $M_{\mathcal{C}}(f, g, t) \geq M_{d_{\mathcal{C}}}(f, g, t)$ whenever $t>1$, where $\left(M_{d_{\mathcal{C}}}, \wedge\right)$ is the standard fuzzy quasi-metric induced by $d_{\mathcal{C}}$.

Proof. Let us recall that $M_{d_{\mathcal{C}}}(f, g, t)=t /\left(t+d_{\mathcal{C}}(f, g)\right)$ for all $f, g \in \mathcal{C}$ and $t>0$. Thus by Remark 4.1 we have that $M_{\mathcal{C}}(f, g, t)=M_{d_{\mathcal{C}}}(f, g, t)$ whenever $t \in(0,1]$ and $M_{\mathcal{C}}(f, g, t) \geqslant M_{d_{\mathcal{C}}}(f, g, t)$ whenever $t>1$. Next we show that $\left(M_{\mathcal{C}}, \wedge\right)$ is a fuzzy quasi-metric on $\mathcal{C}$.

Clearly $0<M_{\mathcal{C}}(f, g, t) \leqslant 1$ for all $f, g \in \mathcal{C}$ and $t>0$. In order to show condition(ii) of a fuzzy quasi-metric, let $f, g \in \mathcal{C}$ such that $M_{\mathcal{C}}(f, g, t)=$ 
$M_{\mathcal{C}}(g, f, t)=1$ for all $t>0$, and hence $D_{\mathcal{C}}(f, g, 1)=D_{\mathcal{C}}(g, h, 1)=0$. By Remark $4.1 d_{\mathcal{C}}(f, g)=d_{\mathcal{C}}(g, f)=0$, so $f=g$. Moreover, given $f \in \mathcal{C}$ and $t>0$, it is clear that $M_{\mathcal{C}}(f, f, t)=1$ because $D_{\mathcal{C}}(f, f, t)=0$.

Now let $f, g, h \in \mathcal{C}$ and $t, s>0$. We want to show that $M_{\mathcal{C}}(f, g, t+$ $s) \geq M_{\mathcal{C}}(f, h, t) \wedge M_{\mathcal{C}}(h, g, s)$. Assume, without loss of generality, that $M_{\mathcal{C}}(f, h, t) \leqslant M_{\mathcal{C}}(h, g, s)$. Then $t D_{\mathcal{C}}(h, g, s) \leqslant s D_{\mathcal{C}}(f, h, t)$. So, by using Lemma 4.1 and the above inequality, we obtain:

$$
t D_{\mathcal{C}}(f, g, t+s) \leqslant t D_{\mathcal{C}}(f, h, t)+t D_{\mathcal{C}}(h, g, s) \leqslant(t+s) D_{\mathcal{C}}(f, h, t)
$$

therefore

$$
M_{\mathcal{C}}(f, g, t+s)=\frac{t+s}{t+s+D_{\mathcal{C}}(f, g, t+s)} \geqslant \frac{t}{t+D_{\mathcal{C}}(f, h, t)}=M_{\mathcal{C}}(f, h, t)
$$

Thus we have shown condition (iii) of a fuzzy quasi-metric.

Finally, for $f, g \in \mathcal{C}$ fixed, it is clear that $M_{\mathcal{C}}\left(f, g,_{-}\right)$is left continuous in $[0, \infty)$, because if $t_{m} \rightarrow t^{-}$, there is an $m_{0}$ such that $D_{\mathcal{C}}\left(f, g, t_{m}\right)=D_{\mathcal{C}}(f, g, t)$ for all $m \geqslant m_{0}$, and thus $\lim _{m} M_{\mathcal{C}}\left(f, g, t_{m}\right)=M_{\mathcal{C}}(f, g, t)$.

We conclude that $\left(M_{\mathcal{C}}, \wedge\right)$ is a fuzzy quasi-metric on $\mathcal{C}$

Corollary 4.1. For each continuous t-norm $*,\left(M_{\mathcal{C}}, *\right)$ is a fuzzy quasimetric on $\mathcal{C}$.

Definition 4.1. The fuzzy quasi-metric $\left(M_{\mathcal{C}}, \wedge\right)$ is said to be the complexity fuzzy quasi-metric and the space $\left(\mathcal{C}, M_{\mathcal{C}}, \wedge\right)$ is said to be the complexity fuzzy quasi-metric space. 
Remark 4.2. Observe that this space provides a suitable model to interpret the asymptotic efficiency of the complexity functions. Indeed, if $f, g \in \mathcal{C}$ satisfy that $f$ is asymptotically more efficient than $g$, there exists $n_{0} \in w$ such that $f(n) \leq g(n)$ for all $n \geq n_{0}$, and then $M_{\mathcal{C}}(f, g, t)=1$ for all $t>n_{0}$. Reciprocally, if we compute the complexity fuzzy quasi-metric in $f, g, t$ and obtain that $M_{\mathcal{C}}(f, g, t)=1$ for $t \in\left(n_{0}, n_{0}+1\right]$, it follows that $f(n) \leq g(n)$ for all $n \geq n_{0}$. Hence if $f \neq g$ we have that $f$ is asymptotically more efficient than $\mathrm{g}$. In this way, we have a model to measure complexity distances that gives more information on the computational process than the model of Schellekens.

Remark 4.3. Note that for each $f, g \in \mathcal{C}$ and $t \in(0,1]$, we have $D_{\mathcal{C}}\left(f, g, t_{m}\right)=$ $d_{\mathcal{C}}(f, g)$. Therefore

$$
M_{\mathcal{C}}(f, g, t)=M_{d_{\mathcal{C}}}(f, g, t)
$$

for all $f, g \in \mathcal{C}$ and $t \in[0,1]$, where $\left(M_{d_{\mathcal{C}}}, \wedge\right)$ is the fuzzy quasi-metric induced by $d_{\mathcal{C}}$.

\subsection{Fuzzy contractive maps and fixed point theorems}

Next we present fixed point theorems that we apply to deduce the solution to the recurrence equation associated to Divide \& Conquer algorithms and Quicksort algorithms, respectively. Let us recall (Section 1.1) that by a contractive map on a quasi-metric space $(X, d)$ we mean a self-map $f$ on $X$ such that $d(f x, f y) \leq k d(x, y)$ for all $x, y \in X$, where $k$ is a constant with $0 \leq k<1$, (the number $k$ is called a contraction constant for $f$ ). 
Definition 4.2 [35, 26]. Let $(X, M, *)$ be a fuzzy quasi-metric space and $f: X \rightarrow X$ a self-map. It is said that $f$ is a fuzzy contractive map if there exists $k \in(0,1)$ such that:

$$
M(f(x), f(y), t) \geq \frac{M(x, y, t)}{M(x, y, t)+k(1-M(x, y, t))},
$$

for all $x, y \in X$ and $t>0$.

Definition 4.3. Let $(X, M, *)$ be a fuzzy quasi-metric space and $f: X \rightarrow X$ a self-map. It is said that $f$ is an (0,1]-fuzzy contractive map if there exists $k \in(0,1)$ such that:

$$
M(f(x), f(y), t) \geq \frac{M(x, y, t)}{M(x, y, t)+k(1-M(x, y, t))},
$$

for all $x, y \in X$ and $t \in(0,1]$.

Theorem 4.2. Let $(X, d)$ be a quasi-metric space and let $(M, *)$ a fuzzy quasi-metric on $X$ satisfying:

$$
M(x, y, t)=\frac{t}{t+d(x, y)}, \quad t \in(0,1] .
$$

Then:

(a) The topologies $\tau_{d}$ and $\tau_{M}$ are the same.

(b) A sequence $\left\{x_{n}\right\}_{n}$ in $X$ is a Cauchy sequence in the metric space $\left(X, d^{s}\right)$ if and only if it is a Cauchy sequence in the fuzzy metric space $\left(X, M^{i}, *\right)$.

(c) The fuzzy quasi-metric space $(X, M, *)$ is bicomplete if and only if the quasi-metric space $(X, d)$ is bicomplete. 
(d) A map $f: X \rightarrow X$ is (0,1]-fuzzy contractive if and only if it is a contractive map on the quasi-metric space $(X, d)$.

Proof.

(a) We have that:

$x_{n} \rightarrow x$ with respect to $\tau_{d}$,

$\Longleftrightarrow d\left(x, x_{n}\right) \rightarrow 0$,

$\Longleftrightarrow M\left(x, x_{n}, t\right) \rightarrow 1$ for each $t \in(0,1]$,

$\Longleftrightarrow x_{n} \rightarrow x$ with respect to $\tau_{M}$. So $\tau_{d}=\tau_{M}$ in $X$.

(b) Let $\left\{x_{n}\right\}_{n}$ be a Cauchy sequence in $\left(X, d^{s}\right)$. Fix $t \in(0,1)$. Let $\varepsilon \in(0,1)$ with $t>1-\varepsilon$. There exists $n_{0} \in \mathbb{N}$ such that $d^{s}\left(x_{n}, x_{m}\right)<\varepsilon$ for all $n, m \geq n_{0}$. So $M\left(x_{n}, x_{m}, t\right)>t /(t+\varepsilon)>1-\varepsilon$ for all $n, m \geq n_{0}$. So, $\left(x_{n}\right)_{n}$ is a Cauchy sequence in $\left(X, M^{i}, *\right)$. Reciprocally if $\left\{x_{n}\right\}_{n}$ is a Cauchy sequence in the fuzzy metric space $\left(X, M^{i}, *\right)$, given $\varepsilon \in(0,1 / 2)$ there exists $n_{0} \in \mathbb{N}$ such that $M^{i}\left(x_{n}, x_{m}, 1\right)>1-\varepsilon$ for all $n, m \geq n_{0}$. So $1 /\left(1+d^{s}\left(x_{n}, x_{m}\right)\right)>1-\varepsilon$ for all $n, m \geq n_{0}$. Hence $d^{s}\left(x_{n}, x_{m}\right)<\varepsilon /(1-\varepsilon)<2 \varepsilon$ for all $n, m \geq n_{0}$. We conclude that $\left(x_{n}\right)_{n}$ is a Cauchy sequence in $\left(X, d^{s}\right)$.

(c) It is follows from (a) and (b).

(d) Let $f: X \rightarrow X$ and $k \in(0,1)$. Then we have that for all $t \in(0,1]$

$$
\frac{M(x, y, t)}{M(x, y, t)+k(1-M(x, y, t))}=\frac{t}{t+k d(x, y)}
$$

and

$$
M(f(x), f(y), t)=\frac{t}{t+d(f(x), f(y))} .
$$

Therefore 


$$
\begin{gathered}
d(f(x), f(y)) \leq k d(x, y) \\
\Leftrightarrow \frac{M(x, y, t)}{M(x, y, t)+k(1-M(x, y, t))} \leq M(f(x), f(y), t),
\end{gathered}
$$

for all $x, y \in X$. This shows that $f$ is an $(0,1]$-fuzzy contractive map on $(X, M, *)$ if and only if it is a contractive map on the quasi-metric space $(X, d)$.

Theorem 4.3. Let $(X, d)$ be a bicomplete quasi-metric space and let $(M, *)$ be a fuzzy quasi-metric on $X$ such that:

$$
M(x, y, t)=\frac{t}{t+d(x, y)}
$$

with $t \in(0,1]$. If $f: X \rightarrow X$ is a $(0,1]$-fuzzy contractive map, then $f$ has a unique fixed point.

Proof. If $f: X \rightarrow X$ is a (0,1]-fuzzy contractive map by Theorem $3.2 f$ is a contractive map on the bicomplete quasi-metric space $(X, d)$, so, it is a contractive map on the complete metric space $\left(X, d^{s}\right)$. Hence, by the Banach fixed point theorem $f$ has a unique fixed point.

\subsection{Applications}

Example 4.2. If $\Phi$ is a functional associated to a Divide $\&$ Conquer algorithm, then 


$$
\Phi(f)(1)=c, \text { and } \Phi(f)(n)=a f(n / b)+h(n)
$$

if $n \in\left\{b^{k}: k \in \mathbb{N}\right\}$, where $a, b, c \in \mathbb{N}, a, b \geq 2$, and $h \in \mathcal{C}$ with $h(n)<\infty$. Then $\Phi$ is a contraction on a closed subspace of the complexity space $\left(\mathcal{C}, d_{\mathcal{C}}\right)$ with contraction constant $k=1 / a$ [50]. We deduce from Theorem 4.2 that $\Phi$ is an (0,1]-fuzzy contraction on a closed subspace of the complexity fuzzy quasi-metric space, with contraction constant 1/a [50]. Since the complexity space $\left(\mathcal{C}, d_{\mathcal{C}}\right)$ is bicomplete [42], by Theorem 4.3, $\Phi$ has a unique fixed point which is obviously the unique solution to the recurrence equation associated to the algorithm.

Example 4.3. Let $T$ be the recurrence equation of a Quicksort algorithm given by:

$$
\begin{aligned}
T(1) & =0, \quad \text { and } \\
T(n) & =\frac{2(n-1)}{n}+\frac{n+1}{n} T(n-1), \quad n \geq 2 .
\end{aligned}
$$

We associate to $T$ the functional $\Phi: \mathcal{C} \rightarrow \mathcal{C}$ given by: $(\Phi(f))_{1}=T(1)$ and

$$
(\Phi(f))_{n}=\frac{2(n-1)}{n}+\frac{n+1}{n} f_{n-1}
$$

for all $n \geq 2$. Next we show that $\Phi$ is a contraction on the bicomplete quasi-metric space $\left(\mathcal{C}, d_{\mathcal{C}}\right)$, with contraction constant $1 / 2$. We have: 


$$
\begin{aligned}
d_{\mathcal{C}}(\Phi f(n), \Phi g(n))= & \sum_{n=1}^{\infty} 2^{-n}\left(\left(\frac{1}{\Phi g(n)}-\frac{1}{\Phi f(n)}\right) \vee 0\right)= \\
& \sum_{n=2}^{\infty} 2^{-n}\left(\left(\frac{1}{\frac{2(n-1)}{n}+\frac{n+1}{n} g_{n-1}}-\frac{1}{\frac{2(n-1)}{n}+\frac{n+1}{n} f_{n-1}}\right) \vee 0\right) \\
\leq & \sum_{n=2}^{\infty} 2^{-n}\left(\left(\frac{n+1}{n}\left(f_{n-1}-g_{n-1}\right)\right.\right. \\
\leq & \sum_{n=2}^{\infty} 2^{-n}\left(\left(\frac{2\left(\frac{2(n-1)}{n}+\frac{n+1}{n} g_{n-1}\right)\left(\frac{2(n-1)}{n}+\frac{n+1}{n} f_{n-1}\right)}{g_{n-1} f_{n-1}}\right) \vee 0\right) \\
\leq & 1 / 2 \sum_{n=1}^{\infty} 2^{-n}\left(\left(\frac{\left(f_{n}-g_{n}\right)}{g_{n} f_{n}}\right) \vee 0\right) \\
\leq & 1 / 2 \sum_{n=1}^{\infty} 2^{-n}\left(\left(\frac{1}{g(n)}-\frac{1}{f(n)}\right) \vee 0\right)=1 / 2 d_{\mathcal{C}}(f(n), g(n))
\end{aligned}
$$

So, by Theorem 4.2, we have that $\Phi$ is an (0,1]-fuzzy contraction on the complexity fuzzy quasi-metric space $\left(\mathcal{C}, M_{\mathcal{C}}, \wedge\right)$, with contraction constant $1 / 2$, so by Theorem 4.3 we conclude that $\Phi$ has a unique fixed point which is, obviously, the unique solution to the recurrence equation $T$ associated to the Quicksort algorithm. 


\section{Chapter 5}

\section{The Banach fixed point theorem in fuzzy quasi-metric spaces with application to the domain of words}

\section{$5.1 \quad$ Introduction}

In this chapter we present a fuzzy quasi-metric version of the Banach contraction principle, which constitutes an extension of the famous Grabiec fixed point theorem. By using this result we show the existence of fixed point for contraction mappings on the domain of words when it is endowed with certain fuzzy quasi-metrics of Baire type. We apply this approach to deduce the existence of solution for some recurrence equations associated to the analysis of Quicksort algorithms and Divide \& Conquer algorithms, respectively.

By using the notion of a fuzzy metric space in the sense of Kramosil and Michalek [30], Grabiec proved in [21] a celebrated fuzzy version of the Banach contraction principle. Although Grabiec's fixed point theorem has 
the disadvantage that it cannot be applied to the fuzzy metric induced by the Euclidean metric on $\mathbb{R}$ (see $[19,59]$ ), we here show that, nevertheless, it provides an efficient tool to obtain fixed points for (fuzzy) contraction mappings on complete non-Archimedean fuzzy metric spaces, and thus it can be applied, for instance, to the domain of words endowed with the fuzzy metric induced by the Baire metric. (Let us recall that the Baire metric provides a suitable mathematical model in denotational semantics of programming languages [3, 4, 5, 29, 34], etc.)

Actually, we will establish our results in the more general framework of fuzzy quasi-metric spaces because, in this context, the measurement of the distance from a word $x$ to another word $y$, automatically indicates if $x$ is a prefix of $y$ or not, while the Baire metric does not provide this information. Finally, we will apply our methods to prove the existence (and uniqueness) of solution for some recurrence equations associated to the asymptotic complexity analysis of Quicksort algorithms and Divide \& Conquer algorithms, respectively.

The main results of this chapter have been published in [41].

\subsection{The Banach fixed point theorem in fuzzy quasi-metric spaces}

In this section we extend Grabiec's fuzzy version of the Banach fixed point theorem to fuzzy quasi-metric spaces.

According to [54], a B-contraction on a fuzzy metric space $(X, M, *)$ is a self-map $f$ on $X$ such that there is a constant $k \in(0,1)$ satisfying

$$
M(f(x), f(y), k t) \geq M(x, y, t)
$$

for all $x, y \in X, t>0$.

In [21], M. Grabiec introduced the notions of G-Cauchy sequence (Definition 1.5) and G-complete fuzzy metric space (Definition 1.6) in order to 
obtain a fuzzy version of the classical Banach fixed point theorem (Theorem $1.2)$.

Generalizing in a natural way the notions of B-contraction to fuzzy quasimetric spaces we introduce the following concept.

Definition 5.1. A B-contraction on a fuzzy quasi-metric space $(X, M, *)$ is a self-map $f$ on $X$ such that there is a constant $k \in] 0,1[$ satisfying

$$
M(f(x), f(y), k t) \geq M(x, y, t)
$$

for all $x, y \in X, t>0$. The number $k$ is then called a contraction constant of $f$.

Recall (Definition 1.10) that a sequence $\left\{x_{n}\right\}_{n}$ in a fuzzy quasi-metric space $(X, M, *)$ is called G-Cauchy if it is a G-Cauchy sequence in the fuzzy metric space $\left(X, M^{i}, *\right)$, and that (Definition 1.11) a fuzzy quasi-metric space $(X, M, *)$ is called $\mathrm{G}$-bicomplete if the fuzzy metric space $\left(X, M^{i}, *\right)$ is $\mathrm{G}$ complete (in this case, we say that $(M, *)$ is a G-bicomplete fuzzy quasimetric on $X)$.

Then, Grabiec's theorem can be extended to fuzzy quasi-metric spaces as follows.

Theorem 5.1. Let $(X, M, *)$ be a G-bicomplete fuzzy quasi-metric space such that $\lim _{t \rightarrow \infty} M(x, y, t)=1$ for all $x, y \in X$. Then every B-contraction on $X$ has a unique fixed point.

Proof. Let $f: X \rightarrow X$ be a B-contraction on $X$ with contraction constant $k \in(0,1)$. Then

$$
M(f(x), f(y), k t) \geq M(x, y, t)
$$


for all $x, y \in X, t>0$. It immediately follows that

$$
M^{i}(f(x), f(y), k t) \geq M^{i}(x, y, t)
$$

for all $x, y \in X, t>0$. Hence $f$ is a B-contraction on the G-complete fuzzy metric space $\left(X, M^{i}, *\right)$ and, by Theorem $1.2, f$ has a unique fixed point.

\subsection{G-bicompleteness in non-Archimedean fuzzy quasi-metric spaces}

As we indicated above, G-completeness has the disadvantage that the fuzzy metric induced by the Euclidean metric on $\mathbb{R}$ is not G-complete. This fact motivates Definitions 1.9 and 1.13 respectively.

It is obvious that each G-(bi)complete fuzzy (quasi-)metric space is (bi)complete, but the fuzzy metric induced by the Euclidean metric on $\mathbb{R}$ provides an example (Example 1.2) of a complete non G-complete fuzzy metric [59].

Next we shall show that each (bi)complete non-Archimedean fuzzy (quasi)metric space is G-(bi)complete. Thus, we obtain an interesting class of spaces for which Theorem 5.1 applies.

The notion of a non-Archimedean fuzzy metric space was introduced by Sapena [49]. We give a natural generalization of this concept to the quasimetric setting.

Definition 5.2. A fuzzy quasi-metric space $(X, M, *)$ such that $M(x, y, t) \geq$ $\min \{M(x, z, t), M(z, y, t)\}$ for all $x, y, z, \in X, t>0$, is called a non-Archimedean fuzzy quasi-metric space, and $(M, *)$ is called a non-Archimedean fuzzy quasimetric.

Example 5.1. Let $(X, d)$ be a quasi-metric space. It is immediate to show 
that $(X, d)$ is a non-Archimedean quasi-metric space if and only if $\left(X, M_{d}, \cdot\right)$ is a non-Archimedean fuzzy quasi-metric space.

Lemma 5.1. Each G-Cauchy sequence in a non-Archimedean fuzzy quasimetric space is a Cauchy sequence.

Proof. Let $\left\{x_{n}\right\}_{n}$ be a G-Cauchy sequence in the non-Archimedean fuzzy quasi-metric space $(X, M, *)$. Fix $\varepsilon \in(0,1)$ and $t>0$. Since

$$
\lim _{n \rightarrow \infty} M^{i}\left(x_{n}, x_{n+1}, t\right)=1,
$$

there is $n_{0} \in \mathbb{N}$ such that $M^{i}\left(x_{n}, x_{n+1}, t\right)>1-\varepsilon$ for all $n \geq n_{0}$.

Now let $n, m \geq n_{0}$ with $m>n$. Then $m=n+j$, for some $j \in \mathbb{N}$. So

$$
M^{i}\left(x_{n}, x_{m}, t\right) \geq \min \left\{M^{i}\left(x_{n}, x_{n+1}, t\right), \ldots, M^{i}\left(x_{n+j-1}, x_{n+j}, t\right)\right\}>1-\varepsilon
$$

We conclude that $\left(x_{n}\right)_{n}$ is a Cauchy sequence in $(X, M, *)$.

Theorem 5.2. Each bicomplete non-Archimedean fuzzy quasi-metric space is G-bicomplete.

Proof. Let $\left\{x_{n}\right\}_{n}$ be a G-Cauchy sequence in the bicomplete non-Archimedean fuzzy quasi-metric space $(X, M, *)$. By Lemma $5.1,\left(x_{n}\right)_{n}$ is a Cauchy sequence in $(X, M, *)$. Hence, there is $x \in X$ such that $\lim _{n \rightarrow \infty} M^{i}\left(x, x_{n}, t\right)=1$ for all $t>0$. We conclude that $\left(X, M^{i}, *\right)$ is G-complete, i.e. $(X, M, *)$ is G-bicomplete.

Corollary 5.1. Each complete non-Archimedean fuzzy metric space is Gcomplete.

The following result permits us to construct in an easy way a nonArchimedean fuzzy quasi-metric from a bounded non-Archimedean quasi- 
metric $d$, which is different from the fuzzy quasi-metric induced by $d$. It will be consider in the next section.

Proposition 5.1. Let $d$ be a non-Archimedean quasi-metric on a set $X$ such that $d(x, y) \leq 1$ for all $x, y \in X$. Let

$$
\begin{aligned}
& M_{d 1}(x, y, 0)=0 \quad \text { for all } x, y \in X, \\
& \left.\left.M_{d 1}(x, y, t)=1-d(x, y) \quad \text { for all } x, y \in X \text { and } t \in\right] 0,1\right], \\
& M_{d 1}(x, y, t)=1 \quad \text { for all } x, y \in X \text { and } t>1 .
\end{aligned}
$$

Then the following statements hold.

(1) $\left(M_{d 1}, \wedge\right)$ is a non-Archimedean fuzzy quasi-metric on $X$.

(2) For each $x, y \in X, t \in] 0,1]$ and $\varepsilon \in] 0,1[$ :

$$
M_{d 1}(x, y, t)>1-\varepsilon \Longleftrightarrow d(x, y)<\varepsilon .
$$

(3) $\tau_{M_{d 1}}=\tau_{d}$ and $\tau_{\left(M_{d 1}\right)^{-1}}=\tau_{d^{-1}}$.

(4) A sequence in $X$ is Cauchy in $\left(X,\left(M_{d 1}\right)^{i}, \wedge\right)$ if and only if it is Cauchy in $\left(X, d^{s}\right)$.

(5) $\left(X, M_{d 1}, \wedge\right)$ is G-bicomplete if and only if $(X, d)$ is bicomplete.

\subsection{Application to the domain of words}

Let $\Sigma$ be a nonempty alphabet. Let $\Sigma^{\infty}$ be the set of all finite and infinite sequences ("words") over $\Sigma$, where we adopt the convention that the empty sequence $\phi$ is an element of $\Sigma^{\infty}$. Denote by $\sqsubseteq$ the prefix order on $\Sigma^{\infty}$, i.e. $x \sqsubseteq y \Leftrightarrow x$ is a prefix of $y$.

Now, for each $x \in \Sigma^{\infty}$ denote by $\ell(x)$ the length of $x$. Then $\ell(x) \in[1, \infty]$ whenever $x \neq \phi$ and $\ell(\phi)=0$. For each $x, y \in \Sigma^{\infty}$ let $x \sqcap y$ be the common prefix of $x$ and $y$.

Thus, the function $d_{\sqsubseteq}$ defined on $\Sigma^{\infty} \times \Sigma^{\infty}$ by

$$
d_{\sqsubseteq}(x, y)=0 \quad \text { if } x \sqsubseteq y,
$$




$$
d_{\sqsubseteq}(x, y)=2^{-\ell(x \sqcap y)} \quad \text { otherwise, }
$$

is a quasi-metric on $\Sigma^{\infty}$. (We adopt the convention that $2^{-\infty}=0$ ).

Actually $d_{\sqsubseteq}$ is a non-Archimedean quasi-metric on $\Sigma^{\infty}$ (see, for instance, [32] Example 8 (b)).

We also observe that the non-Archimedean metric $\left(d_{\sqsubseteq}\right)^{s}$ is the Baire metric on $\Sigma^{\infty}$, i.e.

$$
\left(d_{\sqsubseteq}\right)^{s}(x, x)=0
$$

and

$$
\left(d_{\sqsubseteq}\right)^{s}(x, y)=2^{-\ell(x \sqcap y)}
$$

for all $x, y \in \Sigma^{\infty}$ such that $x \neq y$.

It is well known that $\left(d_{\sqsubseteq}\right)^{s}$ is complete. From this fact it clearly follows that $d_{\sqsubseteq}$ is bicomplete.

The quasi-metric $d_{\sqsubseteq}$, which was introduced by Smyth [57], will be called the Baire quasi-metric. Observe that condition $d_{\sqsubseteq}(x, y)=0$ can be used to distinguish between the case that $x$ is a prefix of $y$ and the remaining cases.

From Example 5.1 and Theorem 5.2 we obtain the following.

Proposition 5.2. $\left(\Sigma^{\infty}, M_{d_{\sqsubseteq}}, \cdot\right)$ is a G-bicomplete non-Archimedean fuzzy quasi-metric space.

Consequently, Theorem 5.1 can be applied to this useful space. Next we construct other examples of bicomplete non-Archimedean fuzzy quasi-metrics on $\Sigma^{\infty}$ that are related to the Baire quasi-metric defined above.

Example 5.2. Let $d$ be a (non-Archimedean) quasi-metric on a set $X$ and let $M_{d \exp }$ be the fuzzy set in $X \times X \times[0, \infty)$ given by $M_{d \exp }(x, y, 0)=0$ and

$$
M_{d \exp }(x, y, t)=\exp \left(-\frac{d(x, y)}{t}\right)
$$


for all $x, y \in X$ and $t>0$. Then $\left(M_{d \exp }, \wedge\right)$ is a (non-Archimedean) fuzzy quasi-metric on $X$ (compare [19] for the fuzzy metric case). Furthermore, it is easy to see that

$$
M_{d \exp }(x, y, t) \leq M_{d}(x, y, t)
$$

for all $x, y \in X$ and $t \geq 0$. It is also immediate to show that $\tau_{M_{d \mathrm{exp}}}=\tau_{d}$ and that $\left(X, M_{d \exp }, \wedge\right)$ is bicomplete if and only if $(X, d)$ is bicomplete.

From Example 5.2 and Theorem 5.2 we obtain the following.

Proposition 5.3. $\left(\Sigma^{\infty}, M_{d_{\sqsubseteq} \exp }, \wedge\right)$ is a G-bicomplete non-Archimedean fuzzy quasi-metric space.

On the other hand, since $d_{\sqsubseteq}(x, y) \leq 1$ for all $x, y \in \Sigma^{\infty}$, we deduce from Proposition 5.1 the following.

Proposition 5.4. $\left(\Sigma^{\infty}, M_{d_{\sqsubseteq 1}}, \wedge\right)$ is a G-bicomplete non-Archimedean fuzzy quasi-metric space.

Note (see Proposition 5.1) that the fuzzy non-Archimedean quasi-metric $\left(M_{d_{\sqsubseteq 1}}, \wedge\right)$ is given by

$$
\begin{aligned}
& M_{d_{\sqsubseteq 1}}(x, y, 0)=0 \quad \text { for all } x, y \in \Sigma^{\infty}, \\
& M_{d_{\sqsubseteq 1}}(x, y, t)=1 \quad \text { if } x \text { is a prefix of } y, \text { and } t>0, \\
& \left.\left.M_{d_{\sqsubseteq 1}}(x, y, t)=1-2^{-\ell(x \sqcap y)} \quad \text { if } x \text { is not a prefix of } y, \text { and } t \in\right] 0,1\right], \\
& M_{d_{\sqsubseteq 1}}(x, y, t)=1 \quad \text { if } x \text { is not a prefix of } y, \text { and } t>1 .
\end{aligned}
$$

Example 5.3. Next we apply any of Proposition 5.2 and Theorem 5.1 to the complexity analysis of Quicksort algorithms. The average case analysis of Quicksort is discussed in [31] (see also [16]), where the following recurrence 
equation is obtained:

$$
\begin{aligned}
T(1) & =0, \quad \text { and } \\
T(n) & =\frac{2(n-1)}{n}+\frac{n+1}{n} T(n-1), \quad n \geq 2 .
\end{aligned}
$$

Consider as an alphabet $\Sigma$ the set of nonnegative real numbers, i.e. $\Sigma=$ $[0, \infty)$. We associate to $T$ the functional $\Phi: \Sigma^{\infty} \rightarrow \Sigma^{\infty}$ given by $(\Phi(x))_{1}=$ $T(1)$ and

$$
(\Phi(x))_{n}=\frac{2(n-1)}{n}+\frac{n+1}{n} x_{n-1}
$$

for all $n \geq 2$ (if $x \in \Sigma^{\infty}$ has length $n<\infty$, we write $x:=x_{1} x_{2} \ldots x_{n}$, and if $x$ is an infinite word we write $\left.x:=x_{1} x_{2} \ldots\right)$.

Next we show that $\Phi$ is a B-contraction on the G-bicomplete non-Archimedean fuzzy quasi-metric space $\left(\Sigma^{\infty}, M_{d_{\sqsubseteq}}, \cdot\right)$, with contraction constant $1 / 2$.

To this end, we first note that, by construction, we have $\ell(\Phi(x))=\ell(x)+1$ for all $x \in \Sigma^{\infty}$ (in particular, $\ell(\Phi(x))=\infty$ whenever $\left.\ell(x)=\infty\right)$.

Furthermore, it is clear that $x \sqsubseteq y \Longleftrightarrow \Phi(x) \sqsubseteq \Phi(y)$, and consequently

$$
\Phi(x \sqcap y) \sqsubseteq \Phi(x) \sqcap \Phi(y)
$$

for all $x, y \in \Sigma^{\infty}$. Hence

$$
\ell(\Phi(x \sqcap y)) \leq \ell(\Phi(x) \sqcap \Phi(y))
$$

for all $x, y \in \Sigma^{\infty}$.

From the preceding observations we deduce that if $x$ is a prefix of $y$, then

$$
M_{d_{\sqsubseteq}}(\Phi(x), \Phi(y), t / 2)=M_{d_{\sqsubseteq}}(x, y, t)=1,
$$

and if $x$ is not a prefix of $y$, then

$$
\begin{aligned}
M_{d_{\sqsubseteq}}(\Phi(x), \Phi(y), t / 2) & =\frac{t / 2}{(t / 2)+2^{-\ell(\Phi(x) \sqcap \Phi(y))}} \\
& \geq \frac{t / 2}{(t / 2)+2^{-\ell(\Phi(x \sqcap y))}}=\frac{t / 2}{(t / 2)+2^{-(\ell(x \sqcap y)+1)}} \\
& =\frac{t}{t+2^{-\ell(x \sqcap y)}}=M_{d_{\sqsubseteq}}(x, y, t)
\end{aligned}
$$


for all $t>0$.

Therefore $\Phi$ is a B-contraction on $\left(\Sigma^{\infty}, M_{d_{\sqsubseteq}}, \cdot\right)$ with contraction constant 1/2. So, by Theorem 5.1, $\Phi$ has a unique fixed point $z=z_{1} z_{2} \ldots$, which is obviously the unique solution to the recurrence equation $T$, i.e. $z_{1}=0$ and

$$
z_{n}=\frac{2(n-1)}{n}+\frac{n+1}{n} z_{n-1}
$$

for all $n \geq 2$.

Remark 5.1. Note that in the above procedure we can use $\left(\Sigma^{\infty}, M_{d_{\sqsubseteq \text { exp }}}, \wedge\right)$ instead of $\left(\Sigma^{\infty}, M_{d_{\sqsubseteq}}, \cdot\right)$, to deduce the existence of a unique solution for the recurrence $T$.

Example 5.4. We conclude the chapter by applying our results to the complexity analysis of Divide 6 Conquer algorithms. Recall (see Section 3.3) that Divide 8 Conquer algorithms solve a problem by recursively splitting it into subproblems each of which is solved separately by the same algorithm, after which the results are combined into a solution of the original problem. Thus, the complexity of a Divide $\&$ Conquer algorithm typically is the solution to the recurrence equation given by

$$
\begin{aligned}
T(1) & =c, \quad \text { and } \\
T(n) & =a T\left(\frac{n}{b}\right)+h(n),
\end{aligned}
$$

where $a, b, c \in \mathbb{N}$ with $a, b \geq 2, n$ range over the set $\left\{b^{p}: p=0,1,2, \ldots\right\}$, and $h(n) \geq 0$ for all $n \in \mathbb{N}$.

As in the case of Quicksort algorithm, take $\Sigma=[0, \infty)$ and put

$$
\Sigma^{\mathbb{N}}:=\left\{x \in \Sigma^{\infty}: \ell(x)=\infty\right\}
$$

Clearly $\Sigma^{\mathbb{N}}$ is a closed subset of $\left(\Sigma^{\infty},\left(M_{d_{\sqsubseteq}}\right)^{i}, \cdot\right)$, so $\left(\Sigma^{\mathbb{N}}, M_{d_{\sqsubseteq}}, \cdot\right)$ is a nonArchimedean G-bicomplete fuzzy quasi-metric space by Proposition 5.2. 
Now we associate to $T$ the functional $\Phi: \Sigma^{\mathbb{N}} \rightarrow \Sigma^{\mathbb{N}}$ given by $(\Phi(x))_{1}=$ $T(1)$, and

$$
\begin{aligned}
& (\Phi(x))_{n}=a x_{n / b}+h(n) \quad \text { if } n \in\left\{b^{p}: p=1,2, \ldots\right\}, \text { and } \\
& (\Phi(x))_{n}=0 \text { otherwise. }
\end{aligned}
$$

for all $x \in \Sigma^{\mathbb{N}}$.

For our purposes here it suffices to observe that for each $x, y \in \Sigma^{\mathbb{N}}$, the following inequality holds

$$
\ell(\Phi(x) \sqcap \Phi(y)) \geq 1+\ell(x \sqcap y) .
$$

In fact, if $\ell(x \sqcap y)=0$, then $\ell(\Phi(x) \sqcap \Phi(y)) \geq 1$; and if $b^{p}>\ell(x \sqcap y) \geq$ $b^{p-1}, p \geq 1$, then $b^{p+1}>\ell(\Phi(x) \sqcap \Phi(y)) \geq b^{p}$.

Hence, for each $x, y \in \Sigma^{\mathbb{N}}$ and $t>0$, we obtain

$$
\begin{aligned}
M_{d_{\sqsubseteq}}(\Phi(x), \Phi(y), t / 2) & =\frac{t / 2}{(t / 2)+2^{-\ell(\Phi(x) \sqcap \Phi(y))}} \\
& \geq \frac{t / 2}{(t / 2)+2^{-\ell(x \sqcap y)+1)}}=\frac{t}{t+2^{-\ell(x \sqcap y)}} \\
& =M_{d_{\sqsubseteq}}(x, y, t) .
\end{aligned}
$$

Therefore $\Phi$ is a B-contraction on $\left(\Sigma^{\mathbb{N}}, M_{d_{\sqsubseteq}}, \cdot\right)$ with contraction constant 1/2. So, by Theorem 5.1, $\Phi$ has a unique fixed point $z=z_{1} z_{2} \ldots$.

Consequently, the function $F$ defined on $\left\{b^{p}: p=0,1,2 \ldots\right\}$ by $F\left(b^{p}\right)=z_{b^{p}}$ for all $p \geq 0$, is the unique solution to the recurrence equation of the given Divide \& Conquer algorithm.

Remark 5.2. It is well known that every compact fuzzy metric space is complete (in George and Veeramani's sense). In Chapter 2 it is shown that, in general, a compact fuzzy metric space is not G-complete. Since each complete non-Archimedean fuzzy metric space is G-complete, we have that each compact non-Archimedean fuzzy metric space is G-complete. 


\section{Chapter 6}

\section{Contraction maps on fuzzy quasi-metric spaces and $[0,1]$-fuzzy posets}

\subsection{Introduction}

It is well known ([15]) that each bounded non-Archimedean quasi-metric on a set induces, in a natural way, an [0,1]-fuzzy poset. On the other hand, each $[0,1]$-fuzzy poset can be seen as a stationary non-Archimedean fuzzy quasimetric space for the continuous t-norm $\wedge$. By extending this construction to any continuous t-norm, a stationary fuzzy quasi-metric space is obtained. Motivated by these facts, we present several contraction principles on fuzzy quasi-metric spaces that are applied to the class of spaces described above. Some illustrative examples are also given. Finally, we use our approach to deduce in an easy fashion the existence and uniqueness of solution for the recurrence equations typically associated to the analysis of Probabilistic Divide and Conquer Algorithms.

A quasi-metric $d$ on a set $X$ is said to be bounded if there is $K>0$ such 
that $d(x, y) \leqslant K$ for all $x, y \in X$. In this case we say that $K$ is a bound for $d$.

Recall that continuous t-norms are closely related to basic fuzzy logic $\mathrm{BL}$ as investigated in [27]; in particular, each continuous t-norm induces a (linearly ordered) BL-algebra ([28]).

A fuzzy (quasi-)metric $(M, *)$ on $X$ is called stationary if for each $x, y \in$ $X, M\left(x, y,,_{-}\right)$is a constant function on $(0, \infty)$. In this case we say that $(X, M, *)$ is a stationary fuzzy (quasi-)metric space.

Definition 6.1 ([15],[60]). Let $X$ be a nonempty set and $L$ be a complete lattice. A mapping e $: X \times X \rightarrow L$ is called an L-fuzzy partial order on $X$ if it satisfies the following conditions for all $x, y, z \in X$ :

(i) $e(x, y)=e(y, x)=1$ if and only if $x=y$;

(ii) $e(x, z) \wedge e(z, y) \leqslant e(x, y)$.

An $L$-fuzzy partial ordered set (in short, an $L$-fuzzy poset) is a pair $(X, e)$ such that $X$ is a (nonempty) set and $e$ is an $L$-fuzzy partial order on $X$. If $L=[0,1]$, we say that $(X, e)$ is an $[0,1]$-fuzzy poset.

\subsection{Fuzzy quasi-metric spaces and general- ized $[0,1]$-fuzzy posets}

Let $d$ be a non-Archimedean bounded quasi-metric on a set $X$, with bound $K>0$, then the fuzzy set $e$ in $X \times X$ given by

$$
e(x, y)=1-d(x, y) / K
$$

is a [0,1]-fuzzy partial order on $X$ and hence $(X, e)$ is an [0,1]-fuzzy poset $([15])$. 
Conversely, if $(X, e)$ is an [0,1]-fuzzy poset, then it is clear that $(X, e, \wedge)$ is a stationary non-Archimedean fuzzy quasi-metric space.

In the light of these facts it seems natural to extend the notion of an $[0,1]$ fuzzy poset to any continuous t-norm and establish the relationship between this structure and the notion of a stationary fuzzy quasi-metric space. Thus Definition 6.1 suggests the following notion.

Definition 6.2. A generalized [0,1]-fuzzy partial order on a (nonempty) set $X$ is a pair $(e, *)$ such that $*$ be a continuous $t$-norm and $e$ is a fuzzy set in $X \times X$ such that for all $x, y, z \in X$ :

(i) $e(x, y)=e(y, x)=1$ if and only if $x=y$;

(ii) $e(x, z) * e(z, y) \leqslant e(x, y)$.

By a generalized $[0,1]$-fuzzy poset we mean a triple $(X, e, *)$ such that $X$ is a (nonempty) set and $(e, *)$ is a generalized $[0,1]$-fuzzy partial order on $X$.

Then, the following facts are immediate consequences of Definition 6.2.

Proposition 6.1. Let $d$ be a bounded quasi-metric on a set $X$, with bound $K>0$. Then for each continuous t-norm $*$ such that $* \geqslant *_{L}$, the pair $(e, *)$ is a stationary fuzzy quasi-metric on $X$, where $e$ is the fuzzy set in $X \times X$ given by

$$
e(x, y)=1-\frac{d(x, y)}{K}
$$

for all $x, y \in X$.

Proposition 6.2. Let $(X, e, *)$ be a generalized $[0,1]$-fuzzy poset. Then $(X, e, *)$ is a stationary fuzzy quasi-metric space. 


\subsection{Contraction maps and fixed points}

Fixed point theory of fuzzy metric spaces, in the sense of Kramosil and Michalek, began with the well-known theorem of M. Grabiec [21], which is a nice fuzzy metric version of the Banach contraction principle endowed with a nice contraction condition.

On the other hand, condition $\lim _{t \rightarrow \infty} M(x, y, t)=1$ cause Grabiec's theorem cannot be applied to stationary fuzzy (quasi-)metric spaces, and hence to generalized $[0,1]$-fuzzy posets.

In our next results we shall obtain some contraction map results which avoid these inconveniences of Grabiec's theorem. To this end, the following notions of a Cauchy sequence and of a complete fuzzy quasi-metric space will be suitable.

Definition 6.3. Let $(X, M, *)$ be a fuzzy quasi-metric space. A sequence $\left\{x_{n}\right\}_{n}$ in $X$ is called forward Cauchy if for each $\varepsilon \in(0,1)$ and each $t>0$ there is $n_{0} \in \mathbb{N}$ such that $M\left(x_{n}, x_{m}, t\right)>1-\varepsilon$ whenever $n_{0} \leqslant n \leqslant m$. We say that $(X, M, *)$ is forward complete if every forward Cauchy sequence is convergent with respect to the topology $\tau_{M^{-1}}$, i.e. if there exists $y \in X$ such that $\lim _{n \rightarrow \infty} M\left(x_{n}, y, t\right)=1$ for all $t>0$.

A generalized [0,1]-fuzzy poset $(X, e, *)$ is called complete if it is forward complete as a stationary fuzzy quasi-metric space.

Note that each complete fuzzy metric space is forward complete.

Theorem 6.1. Let $(X, M, *)$ be a forward complete fuzzy quasi-metric space such that $* \geqslant *_{L}$. If $f$ is a self-map on $X$ such that there exist $x_{0} \in X$ and 
$k \in(0,1)$ satisfying

$$
M\left(f^{n} x_{0}, f^{n+1} x_{0}, t\right) \geqslant 1-k+k M\left(f^{n-1} x_{0}, f^{n} x_{0}, t\right)
$$

for all $n \in \mathbb{N}$ and $t>0$, then the sequence $\left(f^{n} x_{0}\right)_{n}$ converges to some $y \in X$ with respect to $\tau_{M^{-1}}$.

If, in addition, $\left(X, \tau_{M^{-1}}\right)$ is a Hausdorff topological space and $f$ is continuous from $\left(X, \tau_{M^{-1}}\right)$ into itself, then $y$ is a fixed point of $f$.

Proof. We first show that $M\left(f^{n} x_{0}, f^{n+1} x_{0}, t\right) \geqslant 1-k^{n}$ for all $n \in \mathbb{N}$ and $t>0$.

Indeed, for $n=1$ we have

$$
M\left(f x_{0}, f^{2} x_{0}, t\right) \geq 1-k+k M\left(x_{0}, f x_{0}\right) \geq 1-k .
$$

So

$$
\begin{aligned}
M\left(f^{2} x_{0}, f^{3} x_{0}, t\right) & \geq 1-k+k M\left(f x_{0}, f^{2} x_{0}, t\right) \\
& \geq 1-k+k(1-k) \\
& =1-k^{2} .
\end{aligned}
$$

Now assume that the inequality holds for $n-1$, with $n>3$. Then

$$
\begin{aligned}
M\left(f^{n} x_{0}, f^{n+1} x_{0}, t\right) & \geqslant 1-k+k M\left(f^{n-1} x_{0}, f^{n} x_{0}, t\right) \\
& \geqslant 1-k+k\left(1-k^{n-1}\right) \\
& =1-k^{n} .
\end{aligned}
$$

Next we show that $\left(f^{n} x_{0}\right)_{n}$ is a forward Cauchy sequence in $(X, M, *)$.

Indeed, for each $n, m \in \mathbb{N}$ (we assume without loss of generality that $m=n+j$ for some $j \in \mathbb{N})$ ), we obtain 


$$
\begin{aligned}
M\left(f^{n} x_{0}, f^{m} x_{0}, t\right) & =M\left(f^{n} x_{0}, f^{n+j} x_{0}, t\right) \\
& \geqslant M\left(f^{n} x, f^{n+1} x, t / j\right) * M\left(f^{n+1} x, f^{n+2} x, t / j\right) * \ldots * M\left(f^{n+j-1} x, f^{n+j} x, t / j\right. \\
& \geqslant\left(1-k^{n}\right) *\left(1-k^{n+1}\right) * \ldots *\left(1-k^{n+j-1}\right) \\
& \geqslant\left(1-k^{n}\right) *_{L}\left(1-k^{n+1}\right) *_{L} \ldots *_{L}\left(1-k^{n+j-1}\right) .
\end{aligned}
$$

Given $\varepsilon>0$, there is $n_{0} \in \mathbb{N}$ such that

$$
\sum_{n=n_{0}}^{\infty} k^{n}<\varepsilon
$$

Therefore, for $n, m \geqslant n_{0}$, with $n=m+j$, it follows that $k^{n}+k^{n+1}+\ldots+$ $k^{n+j-1}<\varepsilon$, and hence

$$
\begin{aligned}
M\left(f^{n} x_{0}, f^{m} x_{0}, t\right) & \geqslant\left(1-k^{n}\right) *_{L}\left(1-k^{n+1}\right) *_{L} \ldots *_{L}\left(1-k^{n+j-1}\right) \\
& =1-\left(k^{n}+k^{n+1}+\ldots+k^{n+j-1}\right) \\
& >1-\varepsilon
\end{aligned}
$$

Consequently $\left(f^{n} x_{0}\right)_{n}$ is a forward Cauchy sequence in $(X, M, *)$. Then, there is $y \in X$ such that $\left(f^{n} x_{0}\right)_{n}$ converges to $y$ with respect to $\tau_{M^{-1}}$.

Finally, if $\left(X, \tau_{M^{-1}}\right)$ is a Hausdorff topological space and $f$ is continuous from $\left(X, \tau_{M^{-1}}\right)$ into itself, then $\left(f^{n} x_{0}\right)_{n}$ converges to $f(y)$ with respect to $\tau_{M^{-1}}$, and by Hausdorffness, $y=f(y)$. The proof is complete.

Corollary 6.1. Let $(X, M, *)$ be a forward complete fuzzy quasi-metric space such that $\left(X, \tau_{M^{-1}}\right)$ is a Hausdorff topological space and $* \geqslant *_{L}$. If $f$ is a self-map on $X$ such that there is $k \in(0,1)$ satisfying

$$
M(f x, f y, t) \geqslant 1-k+k M(x, y, t)
$$

for all $x, y \in X$ and $t>0$, then $f$ has a unique fixed point. 
Corollary 6.2. Let $(X, M, *)$ be a complete fuzzy metric space such that $* \geqslant *_{L}$. If $f$ is a self-map on $X$ such that there is $k \in(0,1)$ satisfying

$$
M(f x, f y, t) \geqslant 1-k+k M(x, y, t)
$$

for all $x, y \in X$ and $t>0$, then $f$ has a unique fixed point.

Next we shall obtain a "G-complete" version of Theorem 6.2 and its corollaries. In this way, a fixed point theorem for [0,1]-fuzzy posets will be derived by virtue of Lemma 5.1 .

Definition 6.4. Let $(X, M, *)$ be a fuzzy quasi-metric space. A sequence $\left\{x_{n}\right\}_{n}$ in $X$ is called forward G-Cauchy if for each $p \in \mathbb{N}$ and each $t>0$, $\lim _{n \rightarrow \infty} M\left(x_{n}, x_{n+p}, t\right)=1$. We say that $(X, M, *)$ is forward $G$-complete if every forward G-Cauchy sequence is convergent with respect to the topology $\tau_{M^{-1}}$

A generalized [0,1]-fuzzy poset $(X, e, *)$ is called G-complete if it is forward G-complete as a stationary fuzzy quasi-metric space.

Note that each G-complete fuzzy metric space is forward G-complete.

Theorem 6.2. Let $(X, M, *)$ be a forward $G$-complete fuzzy quasi-metric space. If $f$ is a self-map on $X$ such that there exist $x_{0} \in X$ and $k \in(0,1)$ satisfying

$$
M\left(f^{n} x_{0}, f^{n+1} x_{0}, t\right) \geqslant 1-k+k M\left(f^{n-1} x_{0}, f^{n} x_{0}, t\right)
$$

for all $n \in \mathbb{N}$ and $t>0$, then the sequence $\left(f^{n} x_{0}\right)_{n}$ converges to some $y \in X$ with respect to $\tau_{M^{-1}}$.

If, in addition, $\left(X, \tau_{M^{-1}}\right)$ is a Hausdorff topological space and $f$ is continuous from $\left(X, \tau_{M^{-1}}\right)$ into itself, then $y$ is a fixed point of $f$. 
Proof. As in the proof of Theorem 6.1 we obtain that $M\left(f^{n} x_{0}, f^{n+1} x_{0}, t\right) \geqslant$ $1-k^{n}$ for all $n \in \mathbb{N}$ and $t>0$.

Next we show that $\left(f^{n} x_{0}\right)_{n}$ is a forward G-Cauchy sequence in $(X, M, *)$.

Fix $p \in \mathbb{N}$. Thus we have:

$$
\begin{aligned}
M\left(f^{n} x_{0}, f^{n+p} x_{0}, t\right) & \geqslant M\left(f^{n} x_{0}, f^{n+1} x_{0}, t / p\right) *(\underline{p}) * M\left(f^{n+p-1} x_{0}, f^{n+p} x_{0}, t / p\right) \\
& \geqslant\left(1-k^{n}\right) * \stackrel{(p)}{*} *\left(1-k^{n+p-1}\right) \\
& \geqslant\left(1-k^{n}\right) * \stackrel{(p)}{\cdots} *\left(1-k^{n}\right)
\end{aligned}
$$

Now given $\varepsilon>0$, there is $n_{0} \in \mathbb{N}$ such that

$$
\left(1-k^{n_{0}}\right) * \stackrel{(p)}{\cdots} *\left(1-k^{n_{0}}\right)>1-\varepsilon
$$

So for $n \geqslant n_{0}$ it follows that

$M\left(f^{n} x_{0}, f^{n+p} x_{0}, t\right) \geqslant\left(1-k^{n}\right) * \stackrel{(p)}{\cdots} *\left(1-k^{n}\right) \geqslant\left(1-k^{n_{0}}\right) * \stackrel{(p)}{\cdots} *\left(1-k^{n_{0}}\right)>1-\varepsilon$

Consequently $\left(f^{n} x_{0}\right)_{n}$ is a forward G-Cauchy sequence in $(X, M, *)$. Then, there is $y \in X$ such that $\left(f^{n} x_{0}\right)_{n}$ converges to $y$ with respect to $\tau_{M^{-1}}$.

Finally, if $\left(X, \tau_{M^{-1}}\right)$ is a Hausdorff topological space and $f$ is continuous from $\left(X, \tau_{M^{-1}}\right)$ into itself, then $\left(f^{n} x_{0}\right)_{n}$ converges to $f(y)$ with respect to $\tau_{M^{-1}}$, and by Hausdorffness, $y=f(y)$. The proof is complete.

Corollary 6.3. Let $(X, M, *)$ be a forward G-complete fuzzy quasi-metric space such that $\left(X, \tau_{M^{-1}}\right)$ is a Hausdorff topological space. If $f$ is a self-map on $X$ such that there is $k \in(0,1)$ satisfying

$$
M(f x, f y, t) \geqslant 1-k+k M(x, y, t)
$$

for all $x, y \in X$ and $t>0$, then $f$ has a unique fixed point. 
Corollary 6.4. Let $(X, M, *)$ be a $G$-complete fuzzy metric space. If $f$ is a self-map on $X$ such that there is $k \in(0,1)$ satisfying

$$
M(f x, f y, t) \geqslant 1-k+k M(x, y, t)
$$

for all $x, y \in X$ and $t>0$, then $f$ has a unique fixed point.

Recall that Lemma 5.1 establishes that each G-Cauchy sequence in a nonArchimedean fuzzy quasi-metric space is a Cauchy sequence.

Theorem 6.3. Let $(X, e)$ be a complete $[0,1]$-fuzzy poset such that $\left(X, \tau_{e^{-1}}\right)$ is a Hausdorff topological space. If $f$ is a self-map on $X$ such that there is $k \in(0,1)$ satisfying

$$
e(f x, f y) \geqslant 1-k+k e(x, y)
$$

for all $x, y \in X$, then $f$ has a unique fixed point.

Proof. We show that $(X, e)$ is G-complete. Indeed, let $\left\{x_{n}\right\}_{n}$ be a GCauchy sequence in the non-Archimedean fuzzy quasi-metric space $(X, e, \wedge)$. By Lemma 5.1, $\left\{x_{n}\right\}_{n}$ is a Cauchy sequence. Since $(X, e)$ is complete, $\left\{x_{n}\right\}_{n}$ converges with respect to $\tau_{e^{-1}}$. Therefore $(X, e)$ is G-complete. Theorem 6.2 concludes the proof.

Corollary 6.5. Let $(X, M, *)$ be a complete non-Archimedean fuzzy metric space. If $f$ is a self-map on $X$ such that there is $k \in(0,1)$ satisfying

$$
M(f x, f y, t) \geqslant 1-k+k M(x, y, t)
$$

for all $x, y \in X$ and $t>0$, then $f$ has a unique fixed point.

Proof. Apply Lemma 5.1 and Corollary 6.4. 


\subsection{Application to recurrence equations}

We conclude the chapter by applying the results obtained in Section 6.3 to show, in a direct and easy way, the existence and uniqueness of solution for the following general recurrence equation

$$
T(n)=p(n)+\sum_{k=1}^{n-1} q(n, k) T(k)
$$

for $n \geqslant 2$, where $T(1) \geqslant 0, p(n)>0$, and $q(n, k)>0$.

Equations of type (1) appear when discussing the analysis of Probabilistic Divide and Conquer Algorithms by means of recurrences (see, for instance, Section 4 of [16]):

Denote by $\Sigma^{\infty}$ the set of all (nonempty) finite and infinite sequences of nonnegative real numbers. If $w$ is a finite sequence, with $w:=w_{1}, w_{2, \ldots}, w_{n}$, we write $\ell(w)=n$, and we say that the length of $w$ is $n$. If $w$ is an infinite sequence we write $\ell(w)=\infty$.

Given $v, w \in \Sigma^{\infty}$, we say that $v$ is a prefix of $w$, and we write $v \sqsubseteq w$, if $\ell(v) \leq \ell(w)$ and $v_{k}=w_{k}$ for all $k \in\{1, \ldots, \ell(v)\}$.

Now define a fuzzy set $M$ in $\Sigma^{\infty} \times \Sigma^{\infty}$ by $M(v, w, t)=1$ if $v=w$ and $t>0 ; M(v, w, t)=1-2^{-\ell(v)}$ if $v \sqsubseteq w$ and $t>0$; and $M(v, w, t)=0$, otherwise.

It is routine to show that $(M, \wedge)$ is a forward G-complete stationary nonArchimedean fuzzy quasi-metric on $\Sigma^{\infty}$ with $\left(X, \tau_{M^{-1}}\right)$ a Hausdorff topological space.

Let $T$ be a recurrence equation of type (1). We associate to $T$ the functional $\Phi_{T}: \Sigma^{\infty} \rightarrow \Sigma^{\infty}$ given by $\left(\Phi_{T} w\right)_{1}=T(1)$ and

$$
\left(\Phi_{T} w\right)_{n}=p(n)+\sum_{k=1}^{n-1} q(n, k) w_{k}
$$

for all $n \geqslant 2$. 
It is clear by the construction that if $\ell(w)=n$ then $\ell\left(\Phi_{T} w\right)=n+1$ (in particular, $\ell\left(\Phi_{T} w\right)=\infty$ whenever $\left.\ell(w)=\infty\right)$.

Moreover, we have that $\Phi_{T} v \sqsubseteq \Phi_{T} w$ whenever $v \sqsubseteq w$. Hence, for $v \sqsubseteq w$ with $\ell(w)=\infty$ we deduce

$$
M\left(\Phi_{T} v, \Phi_{T} w, t\right)=1-2^{-\ell\left(\Phi_{T} v\right)} \geqslant 1-2^{-\ell(v)}=M(v, w, t),
$$

which implies that $\Phi_{T}$ is continuous with respect to $\tau_{M^{-1}}$.

Now let $w$ be the element of $\Sigma^{\infty}$ given by $w:=T(1)$. Then $\ell(w)=1$. Since $w \sqsubseteq \Phi_{T} w$, it follows that $\Phi_{T}^{n} w \sqsubseteq \Phi_{T}^{n+1} w$ for each $n \in \mathbb{N}$, so

$$
M\left(\Phi_{T}^{n} w, \Phi_{T}^{n+1} w, t\right)=1-2^{-\ell\left(\Phi_{T}^{n} w\right)}=2^{-1} M\left(\Phi_{T}^{n-1} w, \Phi_{T}^{n} w, t\right)+2^{-1}
$$

for all $n \in \mathbb{N}$. It follows from Theorem 6.1 (or Theorem 6.2 ), for $k=1 / 2$, that $\Phi_{T}$ has a fixed point which is clearly unique by the construction of $\Phi_{T}$. Hence it is the unique solution of the recurrence $T$. 


\section{Chapter 7}

\section{Some additional remarks on the fixed point theory for fuzzy (quasi-)metric spaces}

\subsection{Introduction}

In this chapter we will establish versions of Corollaries 6.2, 6.4 and 6.5, respectively, in the more general framework of fuzzy quasi-metric spaces. Moreover, we will replace the condition $* \geqslant *_{L}$ by the more general case $* \geqslant *_{n}$, for some $n \in \mathbb{N}$, where $\left\{*_{n}\right\}_{n \in \mathbb{N}}$, (Chapter 1 ) is the $\mathbb{N}$-Yager subclass of continuous t-norms.

We present some examples for which well-known fixed point theorems cannot be applied, and nevertheless, our results can be applied to such examples. 


\subsection{Contraction maps and fixed point theo- rems}

Recall that the $\mathbb{N}$-Yager subclass of continuous t-norms is given by:

$$
a *_{n} b=1-\min \left\{1,\left[(1-a)^{1 / n}+(1-b)^{1 / n}\right]^{n}\right\}
$$

with $n \in \mathbb{N}$.

We modify Corollary 6.2 in the following manner:

Theorem 7.1. Let $(X, M, *)$ be a complete fuzzy metric space such that $* \geqslant *_{\alpha}$, for some $\alpha \in \mathbb{N}$. If $f$ is a self-map on $X$ such that there is $k \in(0,1)$ satisfying

$$
M(f x, f y, t) \geqslant 1-k+k M(x, y, t)
$$

for all $x, y \in X$ and $t>0$, then $f$ has a unique fixed point.

Proof. Fix $x \in X$. We first show that $M\left(f^{n} x, f^{n+1} x, t\right) \geqslant 1-k^{n}$ for all $n \in \mathbb{N}$ and $t>0$.

Indeed, for $n=1$ we have

$$
M\left(f x, f^{2} x, t\right) \geq 1-k+k M(x, f x) \geq 1-k .
$$

So

$$
\begin{aligned}
M\left(f^{2} x, f^{3} x, t\right) & \geq 1-k+k M\left(f x, f^{2} x, t\right) \\
& \geq 1-k+k(1-k) \\
& =1-k^{2} .
\end{aligned}
$$

Now assume that the inequality holds for $n-1$, with $n>3$. Then 


$$
\begin{aligned}
M\left(f^{n} x, f^{n+1} x, t\right) & \geqslant 1-k+k M\left(f^{n-1} x, f^{n} x, t\right) \\
& \geqslant 1-k+k\left(1-k^{n-1}\right) \\
& =1-k^{n} .
\end{aligned}
$$

Next we show that $\left(f^{n} x\right)_{n}$ is a Cauchy sequence in $(X, M, *)$.

Indeed, for each $n, m \in \mathbb{N}$ (we assume without loss of generality that $m=n+j$ for some $j \in \mathbb{N}$ ), we obtain

$$
\begin{gathered}
M\left(f^{n} x, f^{m} x, t\right)=M\left(f^{n} x, f^{n+j} x, t\right) \\
\geqslant M\left(f^{n} x, f^{n+1} x, t / j\right) * M\left(f^{n+1} x, f^{n+2} x, t / j\right) * \ldots * M\left(f^{n+j-1} x, f^{n+j} x, t / j\right) \\
\geqslant\left(1-k^{n}\right) *\left(1-k^{n+1}\right) * \ldots *\left(1-k^{n+j-1}\right) \\
\geqslant\left(1-k^{n}\right) *_{\alpha}\left(1-k^{n+1}\right) *_{\alpha} \ldots *_{\alpha}\left(1-k^{n+j-1}\right) .
\end{gathered}
$$

Given $\varepsilon>0$, there is $n_{0} \in \mathbb{N}$ such that

$$
\sum_{n=n_{0}}^{\infty} k^{n / \alpha}<\varepsilon^{1 / \alpha} .
$$

Therefore, for $n, m \geqslant n_{0}$, with $n=m+j$, it follows that:

$$
k^{n / \alpha}+k^{(n+1) / \alpha}+\ldots+k^{(n+j-1) / \alpha}<\varepsilon^{1 / \alpha},
$$

and hence

$$
\begin{aligned}
M\left(f^{n} x, f^{m} x, t\right) & \geqslant\left(1-k^{n}\right) *_{\alpha}\left(1-k^{n+1}\right) *_{\alpha} \ldots *_{\alpha}\left(1-k^{n+j-1}\right) \\
& =1-\left(k^{n / \alpha}+k^{(n+1) / \alpha}+\ldots+k^{(n+j-1) / \alpha}\right)^{\alpha} \\
& >1-\varepsilon .
\end{aligned}
$$


Consequently $\left(f^{n} x\right)_{n}$ is a Cauchy sequence in $(X, M, *)$. Then, there is $y \in X$ such that $\left(f^{n} x\right)_{n}$ converges to $y$ with respect to $\tau_{M}$.

Since

$$
\begin{aligned}
M\left(f^{n} y, f^{n+1} x, t\right) & \geq 1-k+k M\left(y, f^{n} x, t\right), \quad \text { and } \\
\lim _{n \rightarrow \infty} M\left(y, f^{n} x, t\right) & =1
\end{aligned}
$$

it is follows that

$$
\lim _{n \rightarrow \infty} M\left(f^{n} y, f^{n+1} x, t\right)=1
$$

therefore $\left(f^{n} x\right)_{n}$ converges to $f y$, so $f y=y$.

Finally, suppose that $z \in X$ satisfies $f z=z$, then:

$$
M(y, z, t)=M(f y, f z, t) \geq 1-k+k M(y, z, t),
$$

SO

$$
(1-k) M(y, z, t) \geq(1-k)
$$

and, thus

$$
M(y, z, t)=1
$$

for all $t>0$. We conclude that $z=y$. We have shown that $y$ is the unique fixed point for $f$

In the three next theorems we extend Theorem 7.1 and Corollaries 6.4 and 6.5, respectively, to fuzzy quasi-metrics spaces. 
Theorem 7.2. Let $(X, M, *)$ be a bicomplete fuzzy quasi-metric space such that $* \geqslant *_{\alpha}$, for some $\alpha \in \mathbb{N}$. If $f$ is a self-map on $X$ such that there is $k \in(0,1)$ satisfying

$$
M(f x, f y, t) \geqslant 1-k+k M(x, y, t)
$$

for all $x, y \in X$ and $t>0$, then $f$ has a unique fixed point.

Proof. It immediately follows that

$$
M^{i}(f x, f y, t) \geqslant 1-k+k M^{i}(x, y, t)
$$

for all $x, y \in X$ and $t>0$. Hence $f$ is a contraction in the sense of Theorem 7.1 on the complete fuzzy metric space $\left(X, M^{i}, *\right)$, and, by Theorem $7.1, f$ has a unique fixed point.

Theorem 7.3. Let $(X, M, *)$ be a G-bicomplete fuzzy quasi-metric space. If $f$ is a self-map on $X$ such that there is $k \in(0,1)$ satisfying

$$
M(f x, f y, t) \geqslant 1-k+k M(x, y, t)
$$

for all $x, y \in X$ and $t>0$, then $f$ has a unique fixed point.

Proof. It immediately follows that

$$
M^{i}(f x, f y, t) \geqslant 1-k+k M^{i}(x, y, t)
$$

for all $x, y \in X$ and $t>0$. Hence $f$ is a contraction in the sense of Corollary 6.4 on the G-complete fuzzy metric space $\left(X, M^{i}, *\right)$, and, by Corollary 6.4 , $f$ has a unique fixed point.

Theorem 7.4. Let $(X, M, *)$ be a bicomplete non-Archimedean fuzzy quasimetric space. If $f$ is a self-map on $X$ such that there is $k \in(0,1)$ satisfying 


$$
M(f x, f y, t) \geqslant 1-k+k M(x, y, t)
$$

for all $x, y \in X$ and $t>0$, then $f$ has a unique fixed point.

Proof. It immediately follows that

$$
M^{i}(f x, f y, t) \geqslant 1-k+k M^{i}(x, y, t)
$$

for all $x, y \in X$ and $t>0$. Hence $f$ is a contraction in the sense of Corollary 6.5 on the complete non-Archimedean fuzzy metric space $\left(X, M^{i}, *\right)$, and, by Corollary 6.5, $f$ has a unique fixed point.

Next we present an example where the quasi-metric version of the famous Grabiec's fixed point theorem cannot be applied (Theorem 5.1).

Example 7.1. Proposition 5.4 held that $\left(\Sigma^{\infty}, M_{\left.d_{\sqsubseteq 1}, \wedge\right)}\right.$ is a G-bicomplete non-Archimedean fuzzy quasi-metric space. It is shown in Example 5.3 that $\Phi$, the functional associated to recurrence equation, $T$, of the average case of Quicksort algorithm, has a unique fixed point $z=z_{1} z_{2} \ldots$, which is obviously the unique solution to the recurrence equation $T$, i.e. $z_{1}=0$ and

$$
z_{n}=\frac{2(n-1)}{n}+\frac{n+1}{n} z_{n-1}
$$

for all $n \geq 2$.

Suppose that there exists a contraction constant $k \in(0,1)$ such that

$$
M_{d_{\sqsubseteq}}(\Phi(x), \Phi(y), k t) \geq M_{d_{\sqsubseteq}}(x, y, t),
$$

for all $x, y \in \Sigma^{\infty}$ and $t>0$. Fix $t=1 / k$, then we have:

$$
M_{d_{\sqsubseteq}}(\Phi(x), \Phi(y), 1)=1-2^{-\ell(\Phi(x) \sqcap \Phi(y))} \geq M_{d_{\sqsubseteq}}(x, y, 1 / k)=1,
$$


which is a contradiction. Therefore the conditions of the quasi-metric version of the Grabiec's fixed point theorem (Theorem 5.1) are not satisfied.

Nevertheless, the following example shows that Theorem 7.3 can be applied to deduce the existence of solution for the recurrence equations associated to the analysis of Quicksort algorithms.

Example 7.2. We shall show that $\Phi$ is a contraction (in the sense of Theorem 7.3) on the G-bicomplete fuzzy quasi-metric space $\left(\Sigma^{\infty}, M_{d_{\sqsubseteq 1}}, \wedge\right)$, with contraction constant, $k=1 / 2$.

In Example 5.3 it is shown that $\ell(\Phi(x))=\ell(x)+1$ for all $x \in \Sigma^{\infty}$ (in particular, $\ell(\Phi(x))=\infty$ whenever $\ell(x)=\infty)$. Furthermore $\ell(\Phi(x \sqcap y)) \leq$ $\ell(\Phi(x) \sqcap \Phi(y))$

for all $x, y \in \Sigma^{\infty}$. Then we have:

$$
\begin{aligned}
M_{d_{\sqsubseteq 1}}(\Phi(x), \Phi(y), t) & =1-2^{-\ell(\Phi x \sqcap \Phi y)} \\
& \geq 1-2^{-\ell(\Phi(x \sqcap y))}=1-2^{-(\ell(x \sqcap y)+1)} \\
& =1-\frac{1}{2} 2^{-\ell(x \sqcap y)}=1-\frac{1}{2}+\frac{1}{2} M_{d_{\sqsubseteq 1}}(x, y, t)
\end{aligned}
$$

for all $t \in(0,1]$, and

$$
M_{d_{\sqsubseteq 1}}(\Phi(x), \Phi(y), t)=1 \geq 1-\frac{1}{2}+\frac{1}{2} M_{d_{\sqsubseteq 1}}(x, y, t)=1,
$$

for all $t>1$.

Therefore $\Phi$ is a contraction on $\left(\Sigma^{\infty}, M_{d_{\sqsubseteq}}, \wedge\right)$ with contraction constant 1/2. So, by Theorem 7.3, $\Phi$ has a unique fixed point $z=z_{1} z_{2} \ldots$, which is obviously the unique solution to the recurrence equation $T$, i.e. $z_{1}=0$ and

$$
z_{n}=\frac{2(n-1)}{n}+\frac{n+1}{n} z_{n-1}
$$

for all $n \geq 2$. 
In [26], V. Gregori and A. Sapena gave fixed point theorems for complete GV-fuzzy metric spaces. To this end they introduce the following defintions.

Definition 7.1 [26]. Let $(X, M, *)$ be a $G V$-fuzzy metric space. We will say the map $f: X \rightarrow X$ is fuzzy contractive if there exists $k \in(0,1)$ such that

$$
\frac{1}{M(f x, f y, t)}-1 \leq k\left(\frac{1}{M(x, y, t)}-1\right)
$$

for all $x, y \in X$ and $t>0$. ( $k$ is called the contractive constant of $f$ ).

In [35], Mihet adapted Gregori and Sapena's contractive condition to fuzzy metric spaces (in the sense of Kramosil and Michalek) as follows:

$$
M(f x, f y, t) \geq \frac{M(x, y, t)}{M(x, y, t)+k(1-M(x, y, t))}
$$

for all $x, y \in X$ and $t>0$.

Definition $7.2[26]$. Let $(X, M, *)$ be a $G V$-fuzzy metric space. We will say that the sequence $\left\{x_{n}\right\}_{n}$ in $X$ is fuzzy contractive if there exists $k \in(0,1)$ such that

$$
\frac{1}{M\left(x_{n+1}, x_{n+2}, t\right)}-1 \leq k\left(\frac{1}{M\left(x_{n}, x_{n+1}, t\right)}-1\right)
$$

for all $t>0$ and $n \in \mathbb{N}$.

Following Mihet, we adapte the previous condition to fuzzy metric spaces in the sense of Kramosil and Michalek as follows:

$$
M\left(x_{n+1}, x_{n+2}, t\right) \geq \frac{M\left(x_{n}, x_{n+1}, t\right)}{M\left(x_{n}, x_{n+1}, t\right)+k\left(1-M\left(x_{n}, x_{n+1}, t\right)\right)}
$$


for all $t>0$ and $n \in \mathbb{N}$.

The next theorem was proved by Gregori and Sapena in [26] for GV-fuzzy metric spaces. However the proof remains valid for fuzzy metric spaces in the sense of Kramosil and Michalek.

Theorem 7.5 [26]. Let $(X, M, *)$ be a complete fuzzy metric space in which fuzzy contractive sequences are Cauchy. Let $f: X \rightarrow X$ be a fuzzy contractive map being $k$ the contractive constant. Then $f$ has a unique fixed point.

Gregori and Sapena showed in [26] that each fuzzy contractive sequence is G-Cauchy and asked if a fuzzy contractive sequence is Cauchy. Mihet gave in [35] the following example which shows that the answer is negative in the case of fuzzy metric spaces in the sense of Kramosil and Michalek.

Example $7.3[35]$. Let $X=[0, \infty)$ and $d(x, y)=|x-y|$. Then $(X, d)$ is a complete metric space. In [56] it is shown that $(X, M, *)$ is a complete fuzzy metric space, where $*$ is any continuous t-norm and $M$ is the fuzzy set in $X \times X \times[0,+\infty)$ given in the following way:

$$
\begin{aligned}
& M(x, y, t)=1, \text { if } d(x, y)<t \\
& M(x, y, t)=0, \text { if } d(x, y) \geq t
\end{aligned}
$$

Since

$$
\frac{M(x, y, t)}{M(x, y, t)+k(1-M(x, y, t)}=M(x, y, t),
$$

the contraction condition can be written as:

$$
M(f x, f y, t) \geq M(x, y, t),
$$

for all $x, y \in X$ and $t>0$, or equivalently, 


$$
d(f x, f y) \leq d(x, y)
$$

for all $x, y \in X$. Thus, the map $f: X \rightarrow X, f(x)=x+1$ is a fuzzy contractive map and so every sequence $\left\{x_{n}\right\}_{n}, x_{n}=f^{n}(x)$ is a contractive sequence.

On the other hand, since $f$ is a fixed point free mapping on $(X, M, *)$ it follows from Theorem 7.5 that $\left\{x_{n}\right\}_{n}$ is not a Cauchy sequence.

Next we present an example for which Theorem 7.5 cannot be applied, and nevertheless, Theorem 7.1 works to such an example.

Example 7.4. Let $(X, M, *)$ be a fuzzy metric space, where $*$ is any continuous t-norm satisfying $* \geqslant *_{\alpha}$, for some $\alpha \in \mathbb{N}, X=[0, \infty)$ and $M$ is the fuzzy set in $X \times X \times[0,+\infty)$ given in the following way:

$$
\begin{aligned}
& M(x, y, t)=1, \text { if } d(x, y)<t \\
& M(x, y, t)=0, \text { if } d(x, y) \geq t
\end{aligned}
$$

Let $f: X \rightarrow X$ be the function given by $f(x)=0$ for all $x \in X$. It is obvious that $f$ has a unique fixed point which is $x=0$. Example 7.3 shows that Theorem 7.5 cannot be applied to deduce the existence of the unique fixed point of $f$. On the other hand we have that:

$$
M(f x, f y, t) \geqslant 1-k+k M(x, y, t),
$$

for all $x, y \in X$ and $t>0$, because

$$
M(f x, f y, t)=M(0,0, t)=1 \geqslant 1-k+k M(x, y, t),
$$

for all $x, y \in X$ and $t>0$. Since $(X, M, *)$ is a complete fuzzy metric space and $* \geqslant *_{\alpha}$, for some $\alpha \in \mathbb{N}$, we can applied Theorem 7.1 to deduce the 
existence of the unique fixed point of $f, x=0$.

Remark 7.1. Note that if it is satisfied the contraction in the sense of Theorem 7.1, i.e

$$
M(f x, f y, t) \geqslant 1-k+k M(x, y, t),
$$

for all $x, y \in X$ and $t>0$, then it is satisfied the contraction in the sense of Definition 7.1, i.e

$$
M(f x, f y, t) \geq \frac{M(x, y, t)}{M(x, y, t)+k(1-M(x, y, t)}
$$

for all $x, y \in X$ and $t>0$.

Indeed, if

$$
M(f x, f y, t) \geqslant 1-k+k M(x, y, t),
$$

then

$$
k(1-M(x, y, t)) \geq 1-M(f x, f y, t)
$$

therefore

$$
\frac{k(1-M(x, y, t))}{M(x, y, t)} \geq \frac{1-M(f x, f y, t)}{M(f x, f y, t)},
$$

and so

$$
k\left(\frac{1}{M(x, y, t)}-1\right) \geq \frac{1}{M(f x, f y, t)}-1,
$$

i.e.

$$
\frac{M(x, y, t)}{M(x, y, t)+k(1-M(x, y, t)} \leq M(f x, f y, t)
$$


Gregori and Sapena established in [26] the following theorem:

Theorem 7.6. Let $(X, M, *)$ be a G-complete fuzzy metric space and let $f: X \rightarrow X$ be a fuzzy contractive map. Then $f$ has a unique fixed point.

Remark 7.2. Note that Corollary 6.4 can be also deduced from Theorem 7.6. 


\section{Chapter 8}

\section{On fixed point theorems in intuitionistic fuzzy (quasi-)metric spaces}

\subsection{Introduction}

In [36] J.H. Park introduced and studied a notion of intuitionistic fuzzy metric space that generalizes the concept of fuzzy metric space due to A. George and P. Veeramani [19]. A motivation to this study is the potential applicability of fuzzy intuitionistic topology to quantum particle physics, particularly in connection with both string and $\epsilon^{(\infty)}$ theory developed by M.S. El Naschie [9, 10, 12, 13]. Recently, V. Gregori, S. Romaguera and P. Veeramani have shown [25] that the main metric and topological properties of Park's intuitionistic fuzzy metric spaces can be easily derived from the corresponding ones for fuzzy metric spaces and that such spaces can be motivated from a physic point of view in the context of the two-slit experiment as the foundation of E-infinity of high energy physics, recently studied by El Naschie in $[12,11,13]$. On the other hand, and almost simultaneously, C. Alaca, D. Turkoglu and C. Yildiz [2] have proved intuitionistic fuzzy versions of the celebrated Banach 
fixed point theorem and Edelstein fixed point theorem by using a notion of intuitionistic fuzzy metric space which is based on the concept of fuzzy metric space introduced by I. Kramosil and J. Michalek [30].

Here we show that each intuitionistic fuzzy metric space $(X, M, N, *, \diamond)$ in the sense of $[2]$ generates a (metrizable) topology $\tau_{(M, N)}$ which coincides with the topology $\tau_{M}$ generated by the fuzzy metric space $(X, M, *)$. Furthermore completeness (respectively, compactness) of $(X, M, N, *, \diamond)$ is equivalent to completeness (respectively, compactness) of $(X, M, *)$, and then we deduce that the fixed point theorems obtained in [2] are immediate consequences of the fixed point theorems for fuzzy metric spaces proved by M. Grabiec [21]. Actually, we obtain improved versions of such results and we present an illustrative example.

On the other hand, we show that for each intuitionistic fuzzy metric space $(X, M, N, *, \diamond)$, the topology generated by the "co-fuzzy" metric $N$ in $X$ is also a metrizable topology, and we obtain fixed point theorems by considering completeness of $(X, N, \diamond)$.

Finally we generalize the notions of intuitionistic fuzzy metric space to the quasi-metric setting and we present intuitionistic fuzzy quasi-metric versions of the Banach contraction principle.

The main results of this chapter may be found in [47] and [48].

\subsection{Intuitionistic fuzzy metric spaces and fixed point theorems}

Recently, Alaca, Turkoglu and Yildiz have introduced in [2] the following notion.

Definition 8.1. An intuitionistic fuzzy metric space is a 5-tuple $(X, M, N, *, \diamond)$ 
such that $X$ is a (nonempty) set, $*$ is a continuous t-norm, $\diamond$ is a continuous t-conorm and $M, N$ are fuzzy sets on $X \times X \times[0, \infty)$ satisfying the following conditions, for all $x, y, z \in X$ :

(a) $M(x, y, t)+N(x, y, t) \leq 1$;

(b) $M(x, y, 0)=0$;

(c) $M(x, y, t)=1$ for all $t>0$ if and only if $x=y$;

(d) $M(x, y, t)=M(y, x, t)$ for all $t>0$;

(e) $M(x, y, t) * M(y, z, s) \leq M(x, z, t+s)$ for all $t, s \geq 0$;

(f) $M\left(x, y,{ }_{-}\right):[0, \infty) \rightarrow[0,1]$ is left continuous;

(g) $\lim _{t \rightarrow \infty} M(x, y, t)=1$;

(h) $N(x, y, 0)=1$;

(i) $N(x, y, t)=0$ for all $t>0$ if and only if $x=y$;

(j) $N(x, y, t)=N(y, x, t)$ for all $t>0$;

(k) $N(x, y, t) \diamond N(y, z, s) \geq N(x, z, t+s)$ for all $t, s \geq 0$;

(l) $N\left(x, y,{ }_{-}\right):[0, \infty) \rightarrow[0,1]$ is left continuous.

(m) $\lim _{t \rightarrow \infty} N(x, y, t)=0$.

Remark 8.1. Observe that, in Definition 8.1, condition ( $m$ ) follows immediately from (a) and $(g)$.

Remark 8.2. It is clear that if $(X, M, N, *, \diamond)$ is an intuitionistic fuzzy metric space, then $(X, M, *)$ is a fuzzy metric space. Conversely, if $(X, M, *)$ is a fuzzy metric space, then $(X, M, 1-M, *, \diamond)$ is an intuitionistic fuzzy metric space, where $a \diamond b=1-[(1-a) *(1-b)]$ for all $a, b \in[0,1]$.

Definition $8.2[2]$. Let $\left\{x_{n}\right\}_{n}$ be a sequence in an intuitionistic fuzzy metric space $(X, M, N, *, \diamond)$. Then:

(a) The sequence $\left\{x_{n}\right\}_{n}$ is called a G-Cauchy sequence if for each $p \in \mathbb{N}$ and each $t>0$, we have

$$
\lim _{n \rightarrow \infty} M\left(x_{n+p}, x_{n}, t\right)=1 \quad \text { and } \quad \lim _{n \rightarrow \infty} N\left(x_{n+p}, x_{n}, t\right)=0 .
$$


(b) The sequence $\left\{x_{n}\right\}_{n}$ is convergent to $x \in X$ if for each $t>0$, we have $\lim _{n \rightarrow \infty} M\left(x, x_{n}, t\right)=1 \quad$ and $\quad \lim _{n \rightarrow \infty} N\left(x, x_{n}, t\right)=0$.

Definition 8.3 [2]. An intuitionistic fuzzy metric space $(X, M, N, *, \diamond)$ is called G-complete if every G-Cauchy sequence is convergent.

In order to construct a suitable topology on an intuitionistic fuzzy metric space $(X, M, N, *, \diamond)$ it seems natural to consider "balls" $B(x, r, t)$ defined, similarly to [36], by:

$$
B(x, r, t)=\{y \in X: M(x, y, t)>1-r, N(x, y, t)<r\}
$$

for all $x \in X, r \in(0,1)$ and $t>0$.

Then, one can prove as in [36] that the family of sets of the form $\{B(x, r, t)$ : $x \in X, r \in(0,1), t>0\}$ is a base for a topology $\tau_{(M, N)}$ on $X$.

However, we obtain the following result (compare Proposition 1 of [25]).

Theorem 8.1. Let $(X, M, N, *, \diamond)$ be an intuitionistic fuzzy metric space. Then, for each $x \in X, r \in(0,1), t>0$, we have $B(x, r, t)=B_{M}(x, r, t)$.

Proof. It is clear that $B(x, r, t) \subseteq B_{M}(x, r, t)$.

Now suppose that $y \in B_{M}(x, r, t)$. Then $M(x, y, t)>1-r$, so, by condition (a) of Definition 8.1, we have:

$$
N(x, y, t) \leq 1-M(x, y, t)<1-(1-r)=r .
$$

Consequently $y \in B(x, r, t)$. This concludes the proof.

From Theorem 8.1 we immediately deduce the following results.

Corollary 8.1. Let $(X, M, N, *, \diamond)$ be an intuitionistic fuzzy metric space. Then the topologies $\tau_{(M, N)}$ and $\tau_{M}$ coincide on $X$. 
Corollary 8.2. Let $(X, M, N, *, \diamond)$ be an intuitionistic fuzzy metric space. Then $\left(X, \tau_{(M, N)}\right)$ is a metrizable topological space.

Proposition 8.1 (see [25]). Let $(X, M, N, *, \diamond)$ be an intuitionistic fuzzy metric space. Then the pair $\left(M_{N}, \star\right)$ is a fuzzy metric on $X$ where $M_{N}$ is defined on $X \times X \times[0, \infty)$ by $M_{N}(x, y, t)=1-N(x, y, t)$, and $\star$ is the continuous t-norm defined by $a \star b=1-[(1-a) \diamond(1-b)]$.

In the next section we focus our attention on the structure $(N, \diamond)$, which will be called a co-fuzzy metric.

Corollary 8.3. Let $\left\{x_{n}\right\}_{n}$ be a sequence in an intuitionistic fuzzy metric space $(X, M, N, *, \diamond)$ and let $x \in X$. Then, the following statements are equivalent.

(1) The sequence $\left\{x_{n}\right\}_{n}$ converges to $x$ with respect to $\tau_{(M, N)}$.

(2) The sequence $\left\{x_{n}\right\}_{n}$ converges to $x$ with respect to $\tau_{M}$.

(3) $\lim _{n \rightarrow \infty} M\left(x, x_{n}, t\right)=1$ for all $t>0$.

(4) $\lim _{n \rightarrow \infty} M\left(x, x_{n}, t\right)=1$ and $\lim _{n \rightarrow \infty} N\left(x, x_{n}, t\right)=0$ for all $t>0$.

Proof. (1) $\Longleftrightarrow(2)$. Apply Corollary 8.1.

$(2) \Longleftrightarrow(3)$. This is well known.

$(3) \Longrightarrow(4)$. By hypothesis, for each $r \in(0,1)$ and each $t>0$ there is $n_{0} \in \mathbb{N}$ such that $x_{n} \in B_{M}(x, r, t)$ for all $n \geq n_{0}$, i.e. $x_{n} \in B(x, r, t)$ for all $n \geq n_{0}$, by Theorem 8.1. Hence $N\left(x, x_{n}, t\right)<r$ for all $n \geq n_{0}$. We conclude that $\lim _{n \rightarrow \infty} N\left(x, x_{n}, t\right)=0$ for all $t>0$.

$(4) \Longrightarrow(3)$. Obvious.

Next we deduce the following easy but crucial results.

Proposition 8.2. A sequence in an intuitionistic fuzzy metric space $(X, M, N, *, \diamond)$ is 
G-Cauchy if and only if it is G-Cauchy in the fuzzy metric space $(X, M, *)$.

Proof. Clearly, every G-Cauchy sequence in $(X, M, N, *, \diamond)$ is G-Cauchy in $(X, M, *)$.

Conversely, suppose that $\left\{x_{n}\right\}_{n}$ is a G-Cauchy sequence in $(X, M, *)$. Fix $p \in \mathbb{N}$ and $t>0$. For each $r \in(0,1)$, there is $n_{0} \in \mathbb{N}$ such that $M\left(x_{n+p}, x_{n}, t\right)>1-r$ for all $n \geq n_{0}$. Then

$$
N\left(x_{n+p}, x_{n}, t\right) \leq 1-M\left(x_{n+p}, x_{n}, t\right)<r
$$

for all $n \geq n_{0}$. We conclude that $\lim _{n \rightarrow \infty} N\left(x_{n+p}, x_{n}, t\right)=0$. Therefore $\left(x_{n}\right)_{n}$ is a G-Cauchy sequence in $(X, M, N, *, \diamond)$

Proposition 8.3. An intuitionistic fuzzy metric space $(X, M, N, *, \diamond)$ is $G$ complete if and only if the fuzzy metric space $(X, M, *)$ is $G$-complete.

Proof. Apply Proposition 8.2 and Corollary 8.3, (3) $\Longleftrightarrow 4$.

Corollary 8.4. Let $(X, M, N, *, \diamond)$ be a G-complete intuitionistic fuzzy metric space and let $T: X \rightarrow X$ be a self-map such that

$$
M(T x, T y, k t) \geq M(x, y, t)
$$

for all $x, y \in X$ and $t>0,($ with $k \in(0,1))$. Then $T$ has a unique fixed point.

Proof. Apply Proposition 8.3 and Theorem 1.2.

Corollary 8.5 [2]. Let $(X, M, N, *, \diamond)$ be a G-complete intuitionistic fuzzy metric space and let $T: X \rightarrow X$ be a self-map such that

$$
M(T x, T y, k t) \geq M(x, y, t) \quad \text { and } \quad N(T x, T y, k t) \leq N(x, y, t)
$$

for all $x, y \in X$, and $t>0,($ with $k \in(0,1))$. Then $T$ has a unique fixed point. 
Definition 8.4 [2]. An intuitionistic fuzzy metric space is called compact if every sequence has a convergent subsequence in the sense of Definition 8.2 (b).

Our next result shows that compactness in the sense of Definition 8.4 admits a natural topological characterization.

Proposition 8.4. For an intuitionistic fuzzy metric space $(X, M, N, *, \diamond)$ the following statements are equivalent.

(1) $(X, M, N, *, \diamond)$ is compact.

(2) $(X, M, *)$ is compact.

(3) $\left(X, \tau_{M}\right)$ is a compact topological space.

Proof. (1) $\Longrightarrow(2)$. Obvious.

$(2) \Longrightarrow(3)$. Let $\left\{x_{n}\right\}_{n}$ be a sequence in $X$. By hypothesis, there exist a subsequence $\left\{x_{n_{k}}\right\}_{k}$ of $\left\{x_{n}\right\}_{n}$ and a point $x \in X$ such that $\lim _{k \rightarrow \infty} M\left(x, x_{n_{k}}, t\right)=$ 1 for all $t>0$. By Corollary 8.3, $\left\{x_{n_{k}}\right\}_{k}$ converges to $x$ with respect to $\tau_{M}$. Since $\left(X, \tau_{M}\right)$ is metrizable, we conclude that it is compact.

$(3) \Longrightarrow(1)$. If $\left(X, \tau_{M}\right)$ is compact, then every sequence in $X$ has a convergent subsequence with respect to $\tau_{M}$. Thus, by Corollary $8.3,(2) \Longrightarrow(4)$, it follows that $(X, M, N, *, \diamond)$ is compact.

Corollary 8.6. Let $(X, M, N, *, \diamond)$ be a compact intuitionistic fuzzy metric space and let $T: X \rightarrow X$ be a self-map such that

$$
M(T x, T y, t)>M(x, y, t)
$$

for all $x, y \in X$ with $x \neq y$, and $t>0$. Then $T$ has a unique fixed point.

Proof. Apply Proposition 8.4 and Theorem 1.3.

Corollary $8.7[2]$. Let $(X, M, N, *, \diamond)$ be a compact intuitionistic fuzzy 
metric space and let $T: X \rightarrow X$ be a self-map such that

$$
M(T x, T y, t)>M(x, y, t) \quad \text { and } \quad N(T x, T y, t)<N(x, y, t)
$$

for all $x, y \in X$ with $x \neq y$, and $t>0$. Then $T$ has a unique fixed point.

Next we present an example which shows that, in fact, Corollary 8.4 improves Corollary 8.5 and Corollary 8.6 improves Corollary 8.7.

Example 8.1. Let $X:=\{0\} \cup\{1 / n: n \in \mathbb{N}\}$ and let $d$ be the metric on $X$ given by $d(x, x)=0$ and

$$
d(x, y)=\max \{x, y\}
$$

for all $x, y \in X$ with $x \neq y$. Let $\left(M_{d}, \cdot\right)$ be the fuzzy metric on $X$ induced by $d$ and let $N$ be the fuzzy set in $X \times X \times[0, \infty)$ given by

$$
\begin{aligned}
& N(x, y, t)=1-M_{d}(x, y, t) \quad \text { if } \quad 0 \leq t<1, \quad \text { and } \\
& N(x, y, t)=0 \text { if } t \geq 1 .
\end{aligned}
$$

It is routine to show that $\left(X, M_{d}, N, \cdot, \diamond\right)$ is an intuitionistic fuzzy metric space, where $\diamond$ is the t-conorm associated to $\cdot$, i.e. $a \diamond b=1-((1-a) \cdot(1-b))$ for all $a, b \in[0,1]$.

Since every sequence of distinct points in $X$ converges to 0 with respect to $\tau_{d}$ and $\tau_{d}=\tau_{M}$, it follows that $\left(X, M_{d}, N, \cdot \diamond\right)$ is G-complete and compact.

Now let $T: X \rightarrow X$ be given by $T x=x / 2$ for all $x \in X$. Then, for each $x, y \in X$, with $x \neq y$, and $t>0$ we obtain:

$$
\begin{aligned}
M_{d}(T x, T y, t / 2) & =\frac{t / 2}{(t / 2)+\max \{x / 2, y / 2\}} \\
& =\frac{t}{t+\max \{x, y\}}=M_{d}(x, y, t) .
\end{aligned}
$$

Therefore, the condition of Corollary 8.4 are satisfied for $k=1 / 2$. 
Furthermore, for each $x, y \in X$, with $x \neq y$, and $t>0$ we obtain:

$$
M_{d}(T x, T y, t)=\frac{t}{t+\max \{x / 2, y / 2\}}>\frac{t}{t+\max \{x, y\}}=M_{d}(x, y, t) .
$$

Therefore, the conditions of Corollary 8.6 are satisfied.

Finally, for $x \neq y$ and $k \in(0,1)$, we have $M_{d}(T x, T y, k)<1$, and thus $N(T x, T y, k)>0$. Since $N(T x, T y, 1)=N(x, y, 1)=0$, we conclude that $N(T x, T y, k)>N(x, y, 1)$ and $N(T x, T y, 1)=N(x, y, 1)$.

We have shown that Corollaries 8.5 and 8.7 cannot be applied to the self$\operatorname{map} T$.

Based in the concept of Cauchy sequence and complete fuzzy metric space in the sense of George and Veeramani, Park introduce in [36] the following definitions, which can be given in the sense of Definition 8.1 as follows.

Definition 8.5 [36]. Let $(X, M, N, *, \diamond)$ be an intuitionistic fuzzy metric space. Then

a) a sequence $\left\{x_{n}\right\}_{n}$ in $X$ is said to be Cauchy if for each $\varepsilon>0$ and each $t>0$, there exists $n_{0} \in \mathbb{N}$ such that $M\left(x_{n}, x_{m}, t\right)>1-\varepsilon$ and $N\left(x_{n}, x_{m}, t\right)<$ $\varepsilon$, for all $n, m \geq n_{0}$.

b) $(X, M, N, *, \diamond)$ is called complete if every Cauchy sequence is convergent with respect to $\tau_{(M, N)}$.

Proposition 8.5. A sequence in an intuitionistic fuzzy metric space $(X, M, N, *, \diamond)$ is a Cauchy sequence if and only if it is a Cauchy sequence in the fuzzy metric space $(X, M, *)$.

Proof. Clearly, every Cauchy sequence in $(X, M, N, *, \diamond)$ is a Cauchy sequence in $(X, M, *)$.

Conversely, suppose that $\left\{x_{n}\right\}_{n}$ is a Cauchy sequence in $(X, M, *)$. Fix $\varepsilon \in(0,1)$ and $t>0$; then, there is $n_{0} \in \mathbb{N}$ such that $M\left(x_{n}, x_{m}, t\right)>1-\varepsilon$ for 
all $n, m \geq n_{0}$. Hence

$$
N\left(x_{n}, x_{m}, t\right) \leq 1-M\left(x_{n}, x_{m}, t\right)<\varepsilon
$$

for all $n, m \geq n_{0}$. So $\lim _{n \rightarrow \infty} M\left(x_{n}, x_{m}, t\right)=1$ and $\lim _{n \rightarrow \infty} N\left(x_{n}, x_{m}, t\right)=0$. Therefore $\left(x_{n}\right)_{n}$ is a Cauchy sequence in $(X, M, N, *, \diamond)$

Proposition 8.6. An intuitionistic fuzzy metric space $(X, M, N, *, \diamond)$ is complete if and only if the fuzzy metric space $(X, M, *)$ is complete.

Proof. Apply Proposition 8.5 and Corollary 8.3, (3) $\Longleftrightarrow(4)$

\subsection{Co-fuzzy metric spaces}

Definition 8.6. A co-fuzzy metric space is a triple $(X, N, \diamond)$ such that $X$ is a (nonempty) set, $\diamond$ is a continuous t-conorm and $N$ is a fuzzy set on $X \times X \times[0, \infty)$ satisfying the following conditions, for all $x, y, z \in X$ :

(i) $N(x, y, 0)=1$;

(ii) $N(x, y, t)=0$ for all $t>0$ if and only if $x=y$;

(iii) $N(x, y, t)=N(y, x, t)$ for all $t>0$;

(iv) $N(x, y, t) \diamond N(y, z, s) \geq N(x, z, t+s)$ for all $t, s>0$;

(v) $N\left(x, y,_{-}\right):[0, \infty) \rightarrow[0,1]$ is left continuous,

(vi) $\lim _{t \rightarrow \infty} N(x, y, t)=0$.

If $(X, N, \diamond)$ is a co-fuzzy metric space, we will say that $(N, \diamond)$ (or simply $N)$ is a co-fuzzy metric on $X$.

If $(X, M, N, *, \diamond)$ is an intuitionistic fuzzy metric space, $(X, M, *)$ will be called the associated fuzzy metric space, and $(X, N, \diamond)$ the associated cofuzzy metric space. 
Given an co-fuzzy metric space $(X, N, \diamond)$ we define the open ball $B_{N}(x, r, t)$, for $x \in X, r \in(0,1)$, and $t>0$, as the set $B_{N}(x, r, t)=\{y \in X: N(x, y, t)<$ $r\}$. Obviously, $x \in B_{N}(x, r, t)$.

For each $x \in X, 0<r_{1} \leq r_{2}<1$ and $0<t_{1} \leq t_{2}$, we have $B_{N}\left(x, r_{1}, t_{1}\right) \subseteq$ $B_{N}\left(x, r_{2}, t_{2}\right)$. Consequently, we may define a topology $\tau_{N}$ on $X$ as

$\tau_{N}:=\left\{A \subseteq X:\right.$ for each $x \in A$ there are $r \in(0,1), t>0$, with $\left.B_{N}(x, r, t) \subseteq A\right\}$

Remark 8.3. Let $(X, M, N, *, \diamond)$ be an intuitionistic fuzzy metric space and let $\left(M_{N}, \star\right)$ be the fuzzy metric constructed in Proposition 8.1. Then $\tau_{M_{N}} \subseteq \tau_{M}$, because for each $x \in X, r \in(0,1)$ and $t>0$ we have, by Theorem 8.1, that $B_{M}(x, r, t) \subseteq\{y \in X: N(x, y, t)<r\}=B_{M_{N}}(x, r, t)$. It is obvious that $\tau_{M_{N}}=\tau_{N}$, so $\tau_{N}$ is a metrizable topology on $X$.

Remark 8.4. If $(X, N, \diamond)$ is a co-fuzzy metric space, then $\left(X, M_{N}, N, \star, \diamond\right)$ is an intuitionistic fuzzy metric space, where $a \star b=1-[(1-a) \diamond(1-b)]$ for all $a, b \in[0,1]$, where $M_{N}$ is the fuzzy set of Proposition 8.1.

Proposition 8.7. Let $(X, M, N, *, \diamond)$ be an intuitionistic fuzzy metric space and let $\tau_{N}$ be the topology on $X$ induced by the co-fuzzy metric $(N, \diamond)$. A sequence $\left\{x_{n}\right\}_{n}$ in $X$ converges to $x$ with respect to $\tau_{N}, \lim _{n \rightarrow \infty} x_{n}=0$ if and only if $\lim _{n \rightarrow \infty} N\left(x, x_{n}, t\right)=0$.

Recall that a sequence $\left\{x_{n}\right\}_{n}$ converges to $x$ with respect to $\tau_{(M, N)}$ if and only if $\left\{x_{n}\right\}_{n}$ converges to $x$ with respect to $\tau_{M}$. We also know that $\tau_{N} \subseteq$ $\tau_{(M, N)}=\tau_{M}$, so, the convergence with respect to $\tau_{M}$ implies the convergence 
with respect to $\tau_{N}$.

Next we present an example which shows that the convergence with respect to $\tau_{N}$ does not imply the convergence with respect to $\tau_{(M, N)}$ or $\tau_{M}$, so $\tau_{M} \nsubseteq \tau_{N}$ in general.

Example 8.2. Let $X:=\{0\} \cup\left\{\frac{1}{4 n}: n \in \mathbb{N}\right\}$ and let $d$ be the metric on $X$ given by $d(x, x)=0$ and $d(x, y)=\max \{x, y\}$ for all $x, y \in X$ with $x \neq y$. Define two fuzzy sets, $M$ and $N$ on $X \times X \times[0, \infty)$ by:

$$
\begin{aligned}
& M(x, y, 0)=0, \text { for all } x, y \in X, \\
& M(x, y, t)=\frac{1}{2}-d(x, y) \text { if } x \neq y \text { and } \\
& M(x, y, t)=1 \text { if } x=y, \\
& N(x, y, t)=d(x, y), \text { for all } x, y \in X .
\end{aligned}
$$

Then $(X, M, N, \wedge, \vee)$ is an intuitionistic fuzzy metric space. Let $\left\{x_{n}\right\}_{n}$ be the sequence in $X$ such that $x_{n}=\frac{1}{4 n}$. It is clear that $\lim _{n \rightarrow \infty} N\left(x_{n}, 0, t\right)=0$. Nevertheless $\lim _{n \rightarrow \infty} M\left(x_{n}, 0, t\right)=\frac{1}{2}$.

Definition 8.7. Let $(X, M, N, *, \diamond)$ be an intuitionistic fuzzy metric space. Then

a) a sequence $\left\{x_{n}\right\}_{n}$ in $X$ is said to be $N$-Cauchy if for each $\varepsilon>0$ and each $t>0$, there exists $n_{0} \in \mathbb{N}$ such that $N\left(x_{n}, x_{m}, t\right)<\varepsilon$ for all $n, m \geq n_{0}$.

b) $(X, M, N, *, \diamond)$ is called $N$-complete if every $N$-Cauchy sequence is convergent with respect to $\tau_{N}$.

Every Cauchy sequence in $(X, M, N, *, \diamond)$ is an N-Cauchy sequence, although the opposite is not true as Example 8.2 shows. On the other hand, since $\tau_{N} \subseteq \tau_{(M, N)}=\tau_{M}$, it is easy to see that the completeness of $(X, M, N, *, \diamond)$ and the N-completeness are not related.

Definition 8.8. Let $(X, N, \diamond)$ be a co-fuzzy metric space. A sequence $\left\{x_{n}\right\}_{n}$ 
in $X$ is said to be Cauchy if for each $\varepsilon>0$ and each $t>0$, there exists $n_{0} \in \mathbb{N}$ such that $N\left(x_{n}, x_{m}, t\right)<\varepsilon$ for all $n, m \geq n_{0} . \quad(X, N, \diamond)$ is called complete if every Cauchy sequence is convergent with respect to $\tau_{N}$.

Let $(X, M, N, *, \diamond)$ be an intuitionistic fuzzy metric space, then a sequence $\left\{x_{n}\right\}_{n}$ is an N-Cauchy sequence if and only if it is a Cauchy sequence in the associated co-fuzzy metric space $(X, N, \diamond)$, and the intuitionistic fuzzy metric space $(X, M, N, *, \diamond)$ is $\mathrm{N}$-complete if and only if its associated co-fuzzy metric space $(X, N, \diamond)$ is complete. Moreover, it is easy to see that a sequence $\left\{x_{n}\right\}_{n}$ in $(X, N, \diamond)$ is a Cauchy sequence if and only if it is a Cauchy sequence in $\left(X, M_{N}, \star\right)$.

\subsection{N-contractions and fixed point theorems}

Since $\tau_{M_{N}}=\tau_{N}$, we have that an intuitionistic fuzzy metric space $(X, M, N, *, \diamond)$ is $\mathrm{N}$-complete if and only if its associated co-fuzzy metric space $(X, N, \diamond)$ is complete, if and only if the fuzzy metric space $\left(X, M_{N}, \star\right)$ is complete, if and only if the intuitionistic fuzzy metric space $\left(X, M_{N}, N, \star, \diamond\right)$ is complete. So, we can give some fixed point theorems satisfaying contraction conditions with respect to $N$.

Theorem 8.2. Let $(X, M, N, *, \diamond)$ be an $N$-complete intuitionistic fuzzy metric space such that $\diamond \leq \nabla_{n}$, for some $n \in \mathbb{N}$, and let $T: X \rightarrow X$ a self-map satisfying

$$
N(T x, T y, t) \leq k N(x, y, t)
$$

for all $x, y \in X$ and $t>0$ (with $k \in(0,1)$ ). Then $T$ has a unique fixed point. 
Proof. It is clear that $\left(X, M_{N}, \star\right)$ is a complete fuzzy metric space satisfaying $\star \geq *_{n}$, for some $n \in \mathbb{N}$. Moreover, the self mapping $T$ satisfies

$$
M_{N}(T x, T y, t) \geqslant 1-k+k M_{N}(x, y, t)
$$

for all $x, y \in X$ and $t>0$. By Theorem 7.1, $T$ has a unique fixed point.

Then, by Theorem 7.1 and Theorem 8.2 we have the following theorem

Theorem 8.3. Let $(X, M, N, *, \diamond)$ be an intuitionistic fuzzy metric space, and let $T: X \rightarrow X$ a self-map satisfying

$$
\text { (1) } M(T x, T y, t) \geq 1-k+k M(x, y, t)
$$

or

$$
\text { (2) } N(T x, T y, t) \leq k N(x, y, t)
$$

for all $x, y \in X$ and $t>0$ (with $k \in(0,1)$ ). If $(X, M, N, *, \diamond)$ is a complete intuitionistic fuzzy metric space satisfying (1), with $* \geq *_{n}$, for some $n \in \mathbb{N}$ or, if $(X, M, N, *, \diamond)$ is a $N$-complete intuitionistic fuzzy metric space satisfying (2), with $\diamond \leq \diamond_{n}$, for some $n \in \mathbb{N}$, then $T$ has a unique fixed point.

Definition 8.9. Let $(X, M, N, *, \diamond)$ be an intuitionistic fuzzy metric space. Then

a) a sequence $\left\{x_{n}\right\}_{n}$ in $X$ is said to be $N G$-Cauchy if for each $\varepsilon>0$, $p \in \mathbb{N}$ and $t>0$, there exists $n_{0} \in \mathbb{N}$ such that $N\left(x_{n}, x_{n+p}, t\right)<\varepsilon$ for all $n \geq n_{0}$.

b) $(X, M, N, *, \diamond)$ is called $N G$-complete if every $N G$-Cauchy sequence is convergent with respect to $\tau_{N}$.

Every G-Cauchy sequence is an NG-Cauchy sequence, although the opposite is not true. On the other hand, it is easy to see that the G-completeness 
and the NG-completeness are not related.

Definition 8.10. Let $(X, N, \diamond)$ be a co-fuzzy metric space. A sequence $\left\{x_{n}\right\}_{n}$ in $X$ is said to be G-Cauchy if for each $\varepsilon>0, p \in \mathbb{N}$ and each $t>0$, there exists $n_{0} \in \mathbb{N}$ such that $N\left(x_{n}, x_{n+p}, t\right)<\varepsilon$ for all $n \geq n_{0} .(X, N, \diamond)$ is called $G$-complete if every G-Cauchy sequence is convergent with respect to $\tau_{N}$.

Let $(X, M, N, *, \diamond)$ be an intuitionistic fuzzy metric space, then a sequence $\left\{x_{n}\right\}_{n}$ is a NG-Cauchy sequence if and only if it is a G-Cauchy sequence in the associated co-fuzzy metric space $(X, N, \diamond)$, and the intuitionistic fuzzy metric space $(X, M, N, *, \diamond)$ is NG-complete if and only if its associated cofuzzy metric space $(X, N, \diamond)$ is G-complete. Moreover it is easy to see that a sequence $\left\{x_{n}\right\}_{n}$ in $(X, N, \diamond)$ is a G-Cauchy sequence if and only if it is a G-Cauchy sequence in $\left(X, M_{N}, \star\right)$.

Since $\tau_{M_{N}}=\tau_{N}$, we have that an intuitionistic fuzzy metric space $(X, M, N, *, \diamond)$ is NG-complete if and only if its associated co-fuzzy metric space $(X, N, \diamond)$ is $\mathrm{G}$-complete if and only if the fuzzy metric space $\left(X, M_{N}, \star\right)$ is $\mathrm{G}$-complete if and only if the intuitionistic fuzzy metric space $\left(X, M_{N}, N, \star, \diamond\right)$ is $\mathrm{G}$ complete. So, we give some fixed point theorems satisfaying contraction conditions with respect to $N$.

Theorem 8.4. Let $(X, M, N, *, \diamond)$ be an $N G$-complete intuitionistic fuzzy metric space, and let $T: X \rightarrow X$ a self-map satisfying

$$
N(T x, T y, t) \leq k N(x, y, t)
$$

for all $x, y \in X$ and $t>0$ (with $k \in(0,1)$ ). Then $T$ has a unique fixed point.

Proof. It is clear that $\left(X, M_{N}, \star\right)$ is a $\mathrm{G}$-complete fuzzy metric space. Moreover, the self-map $T$ satisfies 


$$
M_{N}(T x, T y, t) \geqslant 1-k+k M_{N}(x, y, t)
$$

for all $x, y \in X$ and $t>0$. By Corollary $6.4 T$ has a unique fixed point.

Then, by Corollary 6.4 and Theorem 8.4 we have the following theorem

Theorem 8.5. Let $(X, M, N, *, \diamond)$ be an intuitionistic fuzzy metric space, and let $T: X \rightarrow X$ a self-map satisfying

$$
\text { (1) } M(T x, T y, t) \geq 1-k+k M(x, y, t)
$$

or

$$
\text { (2) } N(T x, T y, t) \leq k N(x, y, t)
$$

for all $x, y \in X$ and $t>0$ (with $k \in(0,1)$ ). If $(X, M, N, *, \diamond)$ is a $G$ complete intuitionistic fuzzy metric space satisfying (1), or, if $(X, M, N, *, \diamond)$ is an NG-complete intuitionistic fuzzy metric space satisfying (2), then $T$ has a unique fixed point.

Theorem 8.6. Let $(X, M, N, *, \diamond)$ be an $N G$-complete intuitionistic fuzzy metric space, and let $T: X \rightarrow X$ a self-map satisfying

$$
N(T x, T y, k t) \leq N(x, y, t)
$$

for all $x, y \in X$ and $t>0$ (with $k \in(0,1))$. Then $T$ has a unique fixed point.

Proof. It is clear that $\left(X, M_{N}, \star\right)$ is a G-complete fuzzy metric space, moreover the self-map satisfies

$$
M_{N}(T x, T y, k t) \geq M_{N}(x, y, t)
$$


for all $x, y \in X$ and $t>0$. By Theorem 1.2 $T$ has a unique fixed point.

So, by Corollary 8.4 and Theorem 8.6 we have the following theorem

Theorem 8.7. Let $(X, M, N, *, \diamond)$ be an intuitionistic fuzzy metric space, and let $T: X \rightarrow X$ a self-map satisfying

$$
\text { (1) } M(T x, T y, k t) \geq M(x, y, t)
$$

or

$$
\text { (2) } N(T x, T y, k t) \leq N(x, y, t)
$$

for all $x, y \in X$ and $t>0$ (with $k \in(0,1)$ ). If $(X, M, N, *, \diamond)$ is a $G$ complete intuitionistic fuzzy metric space satisfying (1) or $(X, M, N, *, \diamond)$ is an NG-complete intuitionistic fuzzy metric space satisfying (2), $T$ has a unique fixed point.

The notion of a non-Archimedean fuzzy metric space was introduced by Sapena [49]. We gave in Chapter 5 a natural generalization of this concept to the quasi-metric setting. Next we give a natural generalization of this concept to the intuitionistic fuzzy metric setting.

Definition 8.11. An intuitionistic fuzzy metric space $(X, M, N, *, \diamond)$ such that $(M, *)$ is an non-Archimedean fuzzy metric on $X$ and $N(x, z, t) \leqslant$ $\max \{N(x, y, t), N(y, z, t)\}$ for all $x, y, z \in X, t>0$, is called a non-Archimedean intuitionistic fuzzy metric space, and $(M, N, *, \diamond)$ is called a non-Archimedean intuitionistic fuzzy metric on $X$.

Definition 8.12. A co-fuzzy metric space $(X, N, \diamond)$ such that $N(x, z, t) \leqslant$ $\max \{N(x, y, t), N(y, z, t)\}$ for all $x, y, z \in X, t>0$, is called a non-Archimedean 
co-fuzzy metric space, and $(N, \diamond)$ is called a non-Archimedean co-fuzzy metric on $X$.

It is clear that if $(X, M, *)$ is a non-Archimedean fuzzy metric space, then $(X, M, 1-M, *, \diamond)$ is a non-Archimedan intuitionistic fuzzy metric space, where $a \diamond b=1-[(1-a) *(1-b)]$ for all $a, b \in[0,1]$. Conversely, if $(X, M, N, *, \diamond)$ is a non-Archimedean intuitionistic fuzzy metric space, then $(X, M, *)$ is a non-Archimedean fuzzy metric space. In the same way, if $(X, N, \diamond)$ is a non-Archimedan co-fuzzy metric space, then $\left(X, M_{N}, N, \star, \diamond\right)$ is a non-Archimedean intuitionistic fuzzy metric space, where $a \star b=1-$ $[(1-a) \diamond(1-b)]$ for all $a, b \in[0,1]$. Conversely, if $(X, M, N, *, \diamond)$ is a non-Archimedean intuitionistic fuzzy metric space, then $(X, N, \diamond)$ is a nonArchimedean co-fuzzy metric space.

Theorem 8.8. Let $(X, M, N, *, \diamond)$ be an $N$-complete non-Arquimedean intuitionistic fuzzy metric space, and let $T: X \rightarrow X$ a self-map satisfying

$$
N(T x, T y, t) \leq k N(x, y, t)
$$

for all $x, y \in X$ and $t>0$ (with $k \in(0,1)$ ). Then $T$ has a unique fixed point.

Proof. It is clear that $\left(X, M_{N}, \star\right)$ is a complete non-Arquimedean fuzzy metric space. Moreover, the self-map $T$ satisfies

$$
M_{N}(T x, T y, t) \geqslant 1-k+k M_{N}(x, y, t)
$$

for all $x, y \in X$ and $t>0$. By Corollary $6.5 T$ has a unique fixed point.

Then, by Corollary 6.5 and Theorem 8.8 we have the following theorem

Theorem 8.9. Let $(X, M, N, *, \diamond)$ be a non-Archimedean intuitionistic fuzzy metric space, and let $T: X \rightarrow X$ a self-map satisfying 


$$
\text { (1) } M(T x, T y, t) \geq 1-k+k M(x, y, t)
$$

or

$$
\text { (2) } N(T x, T y, t) \leq k N(x, y, t)
$$

for all $x, y \in X$ and $t>0$ (with $k \in(0,1)$ ). If $(X, M, N, *, \diamond)$ is a complete intuitionistic fuzzy metric space satisfying (1), or, if $(X, M, N, *, \diamond)$ is an $N$-complete intuitionistic fuzzy metric space satisfying (2), then $T$ has a unique fixed point.

Remark 8.5. Note that conditions $(g)$ and $(m)$ are not necesary in the context of Theorems 8.2, 8.3, 8.4, 8.5, 8.8 and 8.9.

We present an example for which Corollary 8.4 cannot be applied to deduce the existence of a unique fixed point, and nevertheless, Theorem 8.7 can be applied.

Example 8.3. Let $X:=\left\{\frac{1}{2 n}: n \in \mathbb{N}\right\}$ and let $d$ be the metric on $X$ given by $d(x, x)=0$ and $d(x, y)=\max \{x, y\}$ for all $x, y \in X$ with $x \neq y$. Define two fuzzy sets, $M$ and $N$ on $X \times X \times[0, \infty)$ by:

$$
\begin{aligned}
& M(x, y, 0)=0, \\
& M(x, y, t)=\frac{1}{2}-d(x, y) \text { if } x \neq y \text { and }, 0<t \leq 1, \\
& M(x, y, t)=1-\frac{d(x, y)}{t} \text { if } x \neq y \text { and }, t>1, \\
& M(x, x, t)=1 \\
& N(x, y, 0)=1 \\
& N(x, y, t)=d(x, y), \text { if } x \neq y \text { and } 0<t \leq 1, \\
& N(x, y, t)=\frac{d(x, y)}{t}, \text { if } x \neq y \text { and } t>1 \\
& N(x, x, t)=0 .
\end{aligned}
$$

It is routine to show that $(X, M, N, \wedge, \vee)$ is an intuitionistic fuzzy metric space. Since every sequence of dictinct points in $X$ conveges to 0 with respec 
to $\tau_{d}$, and $\tau_{N}=\tau_{d}$, it follws that $(X, M, N, \wedge, \vee)$ is $\mathrm{NG-complete.}$

Now let $T: X \rightarrow X$ be given by $T x=x / 2$ for all $x \in X$. An easy computation shows that there is not a $k \in(0,1)$ such that $M(T x, T y, k t) \geq$ $M(x, y, t)$ for all $x, y \in X$ and $t>0$, however $N(T x, T y, t / 2) \leq N(x, y, t)$ for all $x, y \in X$ and $t>0$. Therefore, the conditions of previous theorem are satisfied for $k=1 / 2$. Then $T$ has a unique fixed point which is, obviously, $x=0$.

\subsection{Intuitionistic fuzzy quasi-metric spaces (ifqm- spaces) and fixed point theorems}

We generalize the notions of intuitionistic fuzzy metric space by Alaca, Turkoglu and Yildiz to the quasi-metric setting and we present intuitionistic fuzzy quasi-metric versions of the Banach contraction principle. We apply this approach to deduce the existence of solution for the recurrence equations associated to the analysis of Quicksort algorithm in the framework of intuitionistic fuzzy quasi-metric spaces (ifqm-spaces, in short).

Definition 8.13. An intuitionistic fuzzy quasi-metric space (in the following, an ifqm-space) is a 5-tuple $(X, M, N, *, \diamond)$ such that $X$ is a (nonempty) set, $*$ is a continuous t-norm, $\diamond$ is a continuous t-conorm and $M, N$ are fuzzy sets on $X \times X \times[0, \infty)$ satisfying the following conditions, for all $x, y, z \in X$ :

(a) $M(x, y, t)+N(x, y, t) \leq 1$ for all $t \geq 0$;

(b) $M(x, y, 0)=0$;

(c) $x=y$ if and only if $M(x, y, t)=M(y, x, t)=1$ for all $t>0$;

(d) $M(x, y, t) * M(y, z, s) \leq M(x, z, t+s)$ for all $t, s \geq 0$;

(e) $M\left(x, y,_{-}\right):[0, \infty) \rightarrow[0,1]$ is left continuous; 
(f) $N(x, y, 0)=1$;

(g) $x=y$ if and only if $N(x, y, t)=N(y, x, t)=0$ for all $t>0$;

(h) $N(x, y, t) \diamond N(y, z, s) \geq N(x, z, t+s)$ for all $t, s \geq 0$;

(i) $N\left(x, y,,_{-}\right):[0, \infty) \rightarrow[0,1]$ is left continuous.

In this case we say that $(M, N, *, \diamond)$ is an intuitionistic fuzzy quasi-metric (in the following, an ifqm) on $X$.

If in addition, $M$ and $N$ satisfy that $M(x, y, t)=M(y, x, t)$ and $N(x, y, t)=$ $N(y, x, t)$ for all $x, y \in X$ and $t>0$, then $(M, N, *, \diamond)$ is called an intuitionistic fuzzy metric on $X$ and $(X, M, N, *, \diamond)$ is called an intuitionistic fuzzy metric space.

Note that the authors of [2] require conditions $\lim _{t \rightarrow \infty} M(x, y, t)=1$ and $\lim _{t \rightarrow \infty} N(x, y, t)=0$ in their notion of intuitionistic fuzzy metric space.

Remark 8.6. It is clear that if $(X, M, N, *, \diamond)$ is an ifqm-space, then $(X, M, *)$ is a fuzzy quasi-metric space. Conversely, if $(X, M, *)$ is a fuzzy quasi-metric space, then $(X, M, 1-M, *, \diamond)$ is an ifqm-space, where $a \diamond b=1-[(1-a) *$ $(1-b)]$ for all $a, b \in[0,1]$.

If $(M, N, *, \diamond)$ is an ifqm on $X$, then $\left(M^{-1}, N^{-1}, *, \diamond\right)$ is also an ifqm on $X$, where $M^{-1}$ is the fuzzy set in $X \times X \times[0, \infty)$ defined by $M^{-1}(x, y, t)=$ $M(y, x, t)$ and $N^{-1}$ is the fuzzy set in $X \times X \times[0, \infty)$ defined by $N^{-1}(x, y, t)=$ $N(y, x, t)$. Moreover, if we define $M^{i}$ as above and denote by $N^{s}$ the fuzzy set in $X \times X \times[0, \infty)$ given by $N^{s}(x, y, t)=\max \left\{N(x, y, t), N^{-1}(x, y, t)\right\}$ then $\left(M^{i}, N^{s}, *, \diamond\right)$ is an intuitionistic fuzzy metric on $X$.

In order to construct a suitable topology on an ifqm-space $(X, M, N, *, \diamond)$ it seems natural to consider "balls" $B(x, r, t)$ defined, similarly to [36] and [2], by:

$$
B(x, r, t)=\{y \in X: M(x, y, t)>1-r, N(x, y, t)<r\}
$$


for all $x \in X, r \in(0,1)$ and $t>0$.

Then, one can prove, as in [36], that the family of sets of the form $\{B(x, r, t): x \in X, r \in(0,1), t>0\}$ is a base for a topology $\tau_{(M, N)}$ on $X$.

The following result is analogous to Proposition 1 of [25].

Proposition 8.8. Let $(X, M, N, *, \diamond)$ be an ifqm-space. Then, for each $x \in X, r \in(0,1), t>0$, we have $B(x, r, t)=B_{M}(x, r, t)$.

Proof. It is clear that $B(x, r, t) \subseteq B_{M}(x, r, t)$.

Now suppose that $y \in B_{M}(x, r, t)$. Then $M(x, y, t)>1-r$, so, by condition (a) of Definition 8.10, we have:

$$
N(x, y, t) \leq 1-M(x, y, t)<1-(1-r)=r .
$$

Consequently $y \in B(x, r, t)$. This concludes the proof.

From Proposition 8.8 we immediately deduce the following results.

Corollary 8.8. Let $(X, M, N, *, \diamond)$ be an ifqm-space. Then $\tau_{(M, N)}=\tau_{M}$, $\tau_{\left(M^{-1}, N^{-1}\right)}=\tau_{M^{-1}}$ and $\tau_{\left(M^{i}, N^{s}\right)}=\tau_{M^{i}}$ on $X$.

Corollary 8.9. Let $\left\{x_{n}\right\}_{n}$ be a sequence in an ifqm-space $(X, M, N, *, \diamond)$ and let $x \in X$. Then, the following statements are equivalent.

(1) The sequence $\left\{x_{n}\right\}_{n}$ converges to $x$ with respect to $\tau_{\left(M^{i}, N^{s}\right)}$.

(2) The sequence $\left\{x_{n}\right\}_{n}$ converges to $x$ with respect to $\tau_{M^{i}}$.

(3) $\lim _{n \rightarrow \infty} M^{i}\left(x, x_{n}, t\right)=1$ for all $t>0$.

(4) $\lim _{n \rightarrow \infty} M^{i}\left(x, x_{n}, t\right)=1$ and $\lim _{n \rightarrow \infty} N^{s}\left(x, x_{n}, t\right)=0$ for all $t>0$.

Corollary 8.10. Let $(X, M, N, *, \diamond)$ be an ifqm-space. Then $\left(X, \tau_{(M, N)}\right)$ is a quasi-metrizable topological space and $\left(X, \tau_{\left(M^{i}, N^{s}\right)}\right)$ is a metrizable topological 
space.

Definition 8.14. A co-fuzzy quasi-metric space is a triple $(X, N, \diamond)$ such that $X$ is a (nonempty) set, $\diamond$ is a continuous t-conorm and $N$ is a fuzzy set on $X \times X \times[0, \infty)$ satisfying the following conditions, for all $x, y, z \in X$ :

(i) $N(x, y, 0)=1$;

(ii) $x=y$ if and only if $N(x, y, t)=N(y, x, t)=0$ for all $t>0$;

(iii) $N(x, y, t) \diamond N(y, z, s) \geq N(x, z, t+s)$ for all $t, s \geq 0$;

(iv) $N\left(x, y,_{-}\right):[0, \infty) \rightarrow[0,1]$ is left continuous.

If $(X, N, \diamond)$ is a co-fuzzy quasi-metric space, we will say that $(N, \diamond)$ (or simply $N$ ) is a co-fuzzy quasi-metric on $X$.

If $(X, M, N, *, \diamond)$ is an intuitionistic fuzzy quasi-metric space, we will called to $(X, M, *)$ the associated cfuzzy quasi-metric space, and to $(X, N, \diamond)$ the associated co-fuzzy quasi-metric space.

Given a co-fuzzy quasi-metric space $(X, N, \diamond)$ we define the open ball $B_{N}(x, r, t)$, for $x \in X, r \in(0,1)$, and $t>0$, as the set $B_{N}(x, r, t)=\{y \in X:$ $N(x, y, t)<r\}$. Obviously, $x \in B_{N}(x, r, t)$.

For each $x \in X, 0<r_{1} \leq r_{2}<1$ and $0<t_{1} \leq t_{2}$, we have $B_{N}\left(x, r_{1}, t_{1}\right) \subseteq$ $B_{N}\left(x, r_{2}, t_{2}\right)$. Consequently, we may define a topology $\tau_{N}$ on $X$ as

$\tau_{N}:=\left\{A \subseteq X:\right.$ for each $x \in A$ there are $r \in(0,1), t>0$, with $\left.B_{N}(x, r, t) \subseteq A\right\}$

Proposition 8.9. Let $(X, M, N, *, \diamond)$ be an ifqm-space. Then the pair $\left(M_{N}, \star\right)$ is a fuzzy quasi-metric on $X$ where $M_{N}$ is defined on $X \times X \times[0, \infty)$ by 
$M_{N}(x, y, t)=1-N(x, y, t)$, and $\star$ is the continuous $t$-norm defined by $a \star b=1-[(1-a) \diamond(1-b)]$, for all $a, b \in[0,1]$.

Remark 8.7. Let $(X, M, N, *, \diamond)$ be an ifqm-space and let $\left(M_{N}, \star\right)$ be the fuzzy quasi-metric constructed in Proposition 8.9. Then $\tau_{M_{N}} \subseteq \tau_{M}$, because for each $x \in X, r \in(0,1)$ and $t>0$ we have, by Proposition 8.8, that $B_{M}(x, r, t) \subseteq\{y \in X: N(x, y, t)<r\}=B_{M_{N}}(x, r, t)$. It is obvious that $\tau_{M_{N}}=\tau_{N}$, so $\tau_{N}$ is a quasi-metrizable topology on $X$.

Remark 8.8. It is clear that if $(X, M, N, *, \diamond)$ is an ifqm-space, then $(X, N, \diamond)$ is a co-fuzzy quasi-metric space. Conversely, if $(X, N, \diamond)$ is a co-fuzzy quasi-metric space, then $\left(X, M_{N}, N, \star, \diamond\right)$ is an ifqm-space, where $a \star b=$ $1-[(1-a) \diamond(1-b)]$ for all $a, b \in[0,1]$.

Remark 8.9. If $(X, M, N, *, \diamond)$ is an ifqm-space and $M$ satisfies the symmetry axiom, then $\left(X, M_{1}, N_{1}, *, \diamond_{*}\right)$ is an intuitionistic fuzzy metric space, where $M_{1}=M, N_{1}=1-M$ and $a \diamond_{*} b=1-[(1-a) *(1-b)]$ for all $a, b \in[0,1]$. Moreover $\tau_{M, N}=\tau_{M_{1} N_{1}}$. Reciprocally if $(X, M, N, *, \diamond)$ is an ifqm-space and $N$ satisfies the symmetry axiom then $\left(X, M_{2}, N_{2}, \star, \diamond\right)$ is an intuitionistic fuzzy metric space, where $M_{2}=M_{N}, N_{2}=N$ and $a \star b=1-[(1-a) \diamond(1-b)]$, for all $a, b \in[0,1]$. Moreover $\tau_{N}=\tau_{M_{2} N_{2}} \subseteq \tau_{M, N}$, so, in this case, $\tau_{M, N}$ is a metrizable topology on $X$.

Next we present some fixed point theorems in the framework of ifqmspaces.

Definition 8.15. A sequence $\left\{x_{n}\right\}_{n}$ in an ifqm-space $(X, M, N, *, \diamond)$ is called sequence if for each $\varepsilon \in(0,1), t>0$, there exists $n_{0} \in \mathbb{N}$ such that $M\left(x_{n}, x_{m}, t\right)>$ $1-\varepsilon$, and $N\left(x_{n}, x_{m}, t\right)<\varepsilon$, for all $n, m \geqslant n_{0}$. 
Proposition 8.10. A sequence in an ifqm-space $(X, M, N, *, \diamond)$ is a Cauchy sequence if and only if it is a Cauchy sequence in the fuzzy quasi-metric space $(X, M, *)$.

Proof. Clearly, every Cauchy sequence in $(X, M, N, *, \diamond)$ is a Cauchy sequence in $(X, M, *)$.

Conversely, suppose that $\left\{x_{n}\right\}_{n}$ is a Cauchy sequence in $(X, M, *)$. Fix $\varepsilon \in(0,1)$ and $t>0$; then, there is $n_{0} \in \mathbb{N}$ such that $M^{i}\left(x_{n}, x_{m}, t\right)>1-\varepsilon$ for all $n, m \geq n_{0}$. Hence

$$
N^{s}\left(x_{n}, x_{m}, t\right) \leq 1-M^{i}\left(x_{n}, x_{m}, t\right)<\varepsilon
$$

for all $n, m \geq n_{0}$. So $\lim _{n \rightarrow \infty} M^{i}\left(x_{n}, x_{m}, t\right)=1$ and $\lim _{n \rightarrow \infty} N^{s}\left(x_{n}, x_{m}, t\right)=0$. Therefore $\left\{x_{n}\right\}_{n}$ is a Cauchy sequence in $(X, M, N, *, \diamond)$.

It is proved in Propositio 8.6 that an intuitionistic fuzzy metric space $(X, M, N, *, \diamond)$ is complete if and only if $(X, M, *)$ is complete.

Definition 8.16. An ifqm-space $(X, M, N, *, \diamond)$ is called bicomplete if the intuitionistic fuzzy metric space $\left(X, M^{i}, N^{s}, *, \diamond\right)$ is complete.

Proposition 8.11. An ifqm-space $(X, M, N, *, \diamond)$ is bicomplete if and only if the fuzzy quasi-metric space $(X, M, *)$ is bicomplete.

Definition 8.17. A sequence $\left\{x_{n}\right\}_{n}$ in an ifqm-space $(X, M, N, *, \diamond)$ is called $N$-Cauchy if for each $\varepsilon \in(0,1), t>0$, there exists $n_{0} \in \mathbb{N}$ such that $N\left(x_{n}, x_{m}, t\right)<\varepsilon$, for all $n, m \geqslant n_{0}$.

Definition 8.18. An ifqm-space $(X, M, N, *, \diamond)$ is called $N$-bicomplete if every $N$-Cauchy sequence is convergent with respect to $\tau_{N^{s}}$. 
Definition 8.19. A sequence $\left\{x_{n}\right\}_{n}$ in a co-fuzzy quasi-metric space $(X, N, \diamond)$ is called Cauchy if for each $\varepsilon>0$ and each $t>0$, there exists $n_{0} \in \mathbb{N}$ such that $N\left(x_{n}, x_{m}, t\right)<\varepsilon$ for all $n, m \geq n_{0}$.

Definition 8.20. A co-fuzzy quasi-metric space $(X, N, \diamond)$ is called bicomplete if every Cauchy sequence is convergent with respect to $\tau_{N^{s}}$.

Let $(X, M, N, *, \diamond)$ be an intuitionistic fuzzy quasi-metric space, then a sequence $\left\{x_{n}\right\}_{n}$ is a N-Cauchy sequence if and only if it is a Cauchy sequence in the associated co-fuzzy quasi-metric space $(X, N, \diamond)$, and the intuitionistic fuzzy quasi-metric space $(X, M, N, *, \diamond)$ is N-bicomplete if and only if its associated co-fuzzy quasi-metric space $(X, N, \diamond)$ is bicomplete. Moreover, it is easy to see that a sequence $\left\{x_{n}\right\}_{n}$ in the co-fuzzy quasi-metric spaces $(X, N, \diamond)$ is a Cauchy sequence if and only if it is a Cauchy sequence in its associated fuzzy quasi-metric space $\left(X, M_{N}, \star\right)$.

Since $\tau_{M_{N}}=\tau_{N}$, we have that an intuitionistic fuzzy quasi-metric space $(X, M, N, *, \diamond)$ is N-bicomplete if and only if its associated co-fuzzy quasimetric space $(X, N, \diamond)$ is bi-complete if and only if the fuzzy quasi-metric space $\left(X, M_{N}, \star\right)$ is bicomplete if and only if the intuitionistic fuzzy quasimetric space $\left(X, M_{N}, N, \star, \diamond\right)$ is bicomplete.

Theorem 8.10. Let $(X, M, N, *, \diamond)$ be an intuitionistic fuzzy quasi-metric space, and let $T: X \rightarrow X$ a self map satisfying

$$
\text { (1) } M(T x, T y, t) \geq 1-k+k M(x, y, t)
$$

or

$$
\text { (2) } N(T x, T y, t) \leq k N(x, y, t)
$$

for all $x, y \in X$ and $t>0$ (with $k \in(0,1)$ ). If $(X, M, N, *, \diamond)$ is a bicomplete intuitionistic fuzzy metric space satisfying (1), with $* \geq *_{n}$, for some 
$n \in \mathbb{N}$ or, if $(X, M, N, *, \diamond)$ is a N-bicomplete intuitionistic fuzzy metric space satisfying (2), with $\diamond \leq \nabla_{n}$, for some $n \in \mathbb{N}$, then $T$ has a unique fixed point.

Proof. $\left(X, M^{i}, N^{s}, *, \diamond\right)$ is an intuitionistic fuzzy metric space and $T$ a self-mapping satisfying the conditions of Theorem 8.3 Apply. Theorem 8.3.

Definition 8.21. A sequence $\left\{x_{n}\right\}_{n}$ in an ifqm-space $(X, M, N, *, \diamond)$ is called $G$-Cauchy if $\left\{x_{n}\right\}_{n}$ is a G-Cauchy sequence in $\left(X, M^{i}, N^{s}, *, \diamond\right)$.

Definition 8.22. An ifqm-space $(X, M, N, *, \diamond)$ is called G-bicomplete if $\left(X, M^{i}, N^{s}, *, \diamond\right)$ is a $G$-complete intuitionistic fuzzy metric space.

Proposition 8.12. A sequence in an ifqm-space $(X, M, N, *, \diamond)$ is a $G$ Cauchy sequence if and only if it is a G-Cauchy sequence in the fuzzy quasimetric space $(X, M, *)$.

Proposition 8.13. An ifqm-space $(X, M, N, *, \diamond)$ is G-bicomplete if and only if the fuzzy quasi-metric space $(X, M, *)$ is G-bicomplete.

Definition 8.23. A sequence $\left\{x_{n}\right\}_{n}$ in an ifqm-space $(X, M, N, *, \diamond)$ is called $N G$-Cauchy if $\left\{x_{n}\right\}_{n}$ is an $N G$-Cauchy sequence in $\left(X, M^{i}, N^{s}, *, \diamond\right)$.

Definition 8.24. An ifqm-space $(X, M, N, *, \diamond)$ is called $N G$-bicomplete if $\left(X, M^{i}, N^{s}, *, \diamond\right)$ is an $N G$-complete intuitionistic fuzzy metric space.

Let $(X, N, \diamond)$ be a co-fuzzy quasi-metric space. A sequence $\left\{x_{n}\right\}_{n}$ in $X$ is said to be G-Cauchy if $\left\{x_{n}\right\}_{n}$ is a G-Cauchy sequence in $\left(X, N^{s}, \diamond\right) .(X, N, \diamond)$ is called G-bicomplete if every G-Cauchy sequence is convergent with respect to $\tau_{N^{s}}$. Let $(X, M, N, *, \diamond)$ be an intuitionistic fuzzy quasi-metric space, then 
a sequence $\left\{x_{n}\right\}_{n}$ is a NG-Cauchy sequence if and only if it is a G-Cauchy sequence in the associated co-fuzzy quasi-metric space $(X, N, \diamond)$, and the intuitionistic fuzzy quasi-metric space $(X, M, N, *, \diamond)$ is NG-bicomplete if and only if its associated co-fuzzy quasi-metric space $(X, N, \diamond)$ is G-bicomplete. Moreover, it is easy to see that a sequence $\left\{x_{n}\right\}_{n}$ in the co-fuzzy quasi-metric space $(X, N, \diamond)$ is a G-Cauchy sequence if and only if it is a G-Cauchy sequence in its associated fuzzy quasi-metric space $\left(X, M_{N}, \star\right)$

Since $\tau_{M_{N}}=\tau_{N}$, we have that an intuitionistic fuzzy quasi-metric space $(X, M, N, *, \diamond)$ is NG-bicomplete if and only if its associated co-fuzzy quasimetric space $(X, N, \diamond)$ is G-bicomplete if and only if the fuzzy quasi-metric space $\left(X, M_{N}, \star\right)$ is G-bicomplete if and only if the intuitionistic fuzzy quasimetric space $\left(X, M_{N}, N, \star, \diamond\right)$ is G-bicomplete. So, we give some fixed point theorems satisfaying contraction conditions in $N$ or $M$.

Theorem 8.11. Let $(X, M, N, *, \diamond)$ be an intuitionistic fuzzy quasi-metric space, and let $T: X \rightarrow X$ a self-map satisfying

$$
\text { (1) } M(T x, T y, t) \geq 1-k+k M(x, y, t)
$$

or

$$
\text { (2) } N(T x, T y, t) \leq k N(x, y, t)
$$

for all $x, y \in X$ and $t>0$ (with $k \in(0,1)$ ). If $(X, M, N, *, \diamond)$ is a $G$ bicomplete intuitionistic fuzzy metric space satisfying (1), or, if $(X, M, N, *, \diamond)$ is an NG-bicomplete intuitionistic fuzzy metric space satisfying (2), then $T$ has a unique fixed point.

Proof. $\left(X, M^{i}, N^{s}, *, \diamond\right)$ is an intuitionistic fuzzy metric space and $T$ a self-mapping satisfying the conditons of Theorem 8.5. Apply Theorem 8.5. 
Theorem 8.12. Let $(X, M, N, *, \diamond)$ be an intuitionistic fuzzy quasi-metric space, and let $T: X \rightarrow X$ a self-map satisfying

$$
\text { (1) } M(T x, T y, k t) \geq M(x, y, t) \text { and } \lim _{t \rightarrow \infty} M(x, y, t)=1 \text {, }
$$

or

(2) $N(T x, T y, k t) \leq N(x, y, t)$ and $\lim _{t \rightarrow \infty} N(x, y, t)=0$,

for all $x, y \in X$ and $t>0$ (with $k \in(0,1)$ ). If $(X, M, N, *, \diamond)$ is a $G$ bicomplete intuitionistic fuzzy metric space satisfying (1) or $(X, M, N, *, \diamond)$ is an NG-bicomplete intuitionistic fuzzy metric space satisfying (2), then $T$ has a unique fixed point.

Proof. $\left(X, M^{i}, N^{s}, *, \diamond\right)$ is an intuitionistic fuzzy metric space and $T$ a self-map satisfying the conditons of Theorem 8.7. Apply Theorem 8.7.

The notion of a non-Archimedean fuzzy metric space was introduced by Sapena [49]. We gave in Chapter 4 a natural generalization of this concept to the quasi.metric setting. Nex we give a natural generalization of this concept to the intuitionistic fuzzy quasi-metric setting.

Definition 8.25. An ifqm-space $(X, M, N, *, \diamond)$ such that $(M, *)$ is an nonArchimedean fuzzy quasi-metric on $X$ and $N(x, z, t) \leqslant \max \{N(x, y, t), N(y, z, t)\}$ for all $x, y, z \in X, t>0$, is called a non-Archimedean ifqm-space, and $(M, N, *, \diamond)$ is called a non-Archimedean ifqm on $X$.

Definition 8.26. A co-fuzzy quasi-metric space $(X, N, \diamond)$ such that $N(x, z, t) \leqslant$ $\max \{N(x, y, t), N(y, z, t)\}$ for all $x, y, z \in X, t>0$, is called a non-Archimedan co-fuzzy quasi-metric space, and $(N, \diamond)$ is called a non-Archimedan co-fuzzy quasi-metric on $X$. 
It is clear that if $(X, M, *)$ is a non-Archimedean fuzzy quasi-metric space, then $(X, M, 1-M, *, \diamond)$ is a non-Archimedan intuitionistic fuzzy quasimetric space, where $a \diamond b=1-[(1-a) *(1-b)]$ for all $a, b \in[0,1]$. Conversely, if $(X, M, N, *, \diamond)$ is a non-Archimedean intuitionistic fuzzy quasimetric space, then $(X, M, *)$ is a non-Archimedean fuzzy quasi-metric space. In the same way, if $(X, N, \diamond)$ is a non-Archimedan co-fuzzy quasi-metric space, then $\left(X, M_{N}, N, \star, \diamond\right)$ is a non-Archimedean intuitionistic fuzzy quasimetric space, where $a \star b=1-[(1-a) \diamond(1-b)]$ for all $a, b \in[0,1]$. Conversely, if $(X, M, N, *, \diamond)$ is a non-Archimedean intuitionistic fuzzy quasi-metric space, then $(X, N, \diamond)$ is a non-Archimedean co-fuzzy quasi-metric space.

Theorem 8.13. Let $(X, M, N, *, \diamond)$ be a non-Archimedean intuitionistic fuzzy quasi-metric space, and let $T: X \rightarrow X$ a self-map satisfying

$$
\text { (1) } M(T x, T y, t) \geq 1-k+k M(x, y, t)
$$

or

$$
\text { (2) } N(T x, T y, t) \leq k N(x, y, t)
$$

for all $x, y \in X$ and $t>0$ (with $k \in(0,1)$ ). If $(X, M, N, *, \diamond)$ is a bicomplete intuitionistic fuzzy metric space satisfying (1), or, if $(X, M, N, *, \diamond)$ is an $N$-bicomplete intuitionistic fuzzy metric space satisfying (2), then $T$ has a unique fixed point.

Proof. $\left(X, M^{i}, N^{s}, *, \diamond\right)$ is a non-Archimedean intuitionistic fuzzy metric space and $T$ a self-map satisfying the conditions of Theorem 8.9. Apply Theorem 8.9.

Example 8.4. Let $(X, d)$ be a quasi-metric space. It is immediate to show that $d$ is a non-Archimedean quasi-metric if and only if $\left(M_{d}, 1-M_{d}, \cdot, \diamond\right)$ is 
a non-Archimedean ifqm, where $a \diamond b=1-[(1-a) \cdot(1-b)]$ for all $a, b \in[0,1]$.

The following result, permits us to construct in an easy way a nonArchimedean ifqm from a bounded non-Archimedean quasi-metric $d$, which is different from the ifqm as defined in Example 8.4.

Proposition 8.14. Let $d$ be a non-Archimedean quasi-metric on a set $X$ such that $d(x, y) \leq 1$ for all $x, y \in X$. Let

$$
\begin{aligned}
& M_{d 1}(x, y, 0)=0 \quad \text { for all } x, y \in X, \\
& M_{d 1}(x, y, t)=1-d(x, y) \quad \text { for all } x, y \in X \text { and } t>0, \\
& N_{d 1}(x, y, 0)=1 \quad \text { for all } x, y \in X, \\
& N_{d 1}(x, y, t)=d(x, y) \quad \text { for all } x, y \in X \text { and } t>0,
\end{aligned}
$$

Then the following statements hold.

(1) $\left(M_{d 1}, N_{d 1}, *, \diamond\right)$ is a non-Archimedean ifqm on $X$, where by * we denote any continuous t-norm and by $\diamond$ we denote the t-conorm associated to $*$, and given by $a \diamond b=1-[(1-a) *(1-b)]$, for all $a, b \in[0,1]$

(2) For each $x, y \in X, t \in(0,1)$ and $\varepsilon \in(0,1)$ :

$M(x, y, t)>1-\varepsilon$ and $N(x, y, t)<\varepsilon \Leftrightarrow d(x, y)<\varepsilon \Leftrightarrow M(x, y, t)>1-\varepsilon$

(3) $\tau_{\left(M_{d 1}, N_{d 1}\right)}=\tau_{d}=\tau_{M_{d 1}}$, and $\tau_{\left(\left(M_{d 1}\right)^{-1},\left(N_{d 1}\right)^{-1}\right)}=\tau_{d^{-1}}=\tau_{\left(M_{d 1}\right)^{-1}}$

(4) A sequence in $X$ is Cauchy in $\left(X, M_{d 1}, N_{d 1}, *, \diamond\right)$ if and only if it is Cauchy in $(X, d)$.

(5) $\left(X, M_{d 1}, N_{d 1}, *, \diamond\right)$ is bicomplete if and only if $(X, d)$ is bicomplete.

\subsection{Application to recurrence equations of Quick- sort}

Next we construct an example of bicomplete non-Archimedean ifqm on $\Sigma^{\infty}$ (see Section 5.4) that are related to the Baire quasi-metric defined in Section 
5.4 and for which Theorem 8.13 applies.

Remark 8.10. Let $d_{\sqsubseteq}$ be the Baire quasi-metric on $\Sigma^{\infty}$. Then $d_{\sqsubseteq}$ is a non-Archimedean quasi-metric on $\Sigma^{\infty}$ and $d_{\sqsubseteq}(x, y) \leq 1$ for all $x, y \in \Sigma^{\infty}$. Let

$$
\begin{aligned}
& M_{d_{\sqsubseteq 1}}(x, y, 0)=0 \quad \text { for all } x, y \in \Sigma^{\infty}, \\
& M d_{\sqsubseteq 1}(x, y, t)=1-d_{\sqsubseteq}(x, y) \quad \text { for all } x, y \in \Sigma^{\infty} \text { and } t>0, \\
& N d_{\sqsubseteq 1}(x, y, 0)=1 \quad \text { for all } x, y \in \Sigma^{\infty}, \\
& N d_{\sqsubseteq 1}(x, y, t)=d_{\sqsubseteq}(x, y) \quad \text { for all } x, y \in \Sigma^{\infty} \text { and } t>0 .
\end{aligned}
$$

It follows from Proposition 8.14 that $\left(\Sigma^{\infty}, M_{d_{\sqsubseteq 1}}, N_{d_{\sqsubseteq 1}}, *, \diamond\right)$ is a bicomplete non-Archimedean ifqm-space, where $*$ is any continuous $t$-norm and $\diamond$ is its associated continuous t-conorm.

Next we apply Remark 8.10 and Theorem 8.13 to the complexity analysis of Quicksort algorithm.

Example 8.5. Let $\Phi: \Sigma^{\infty} \rightarrow \Sigma^{\infty}$ be the functional associated to the recurrence equation of the complexity analysis of Quicksort algorithm (Example 5.3). Next we show that $\Phi$ is a contraction (in the sense of Theorem 8.13) on the bicomplete ifqm-space $\left(\Sigma^{\infty}, M_{d_{\sqsubseteq}}, N_{d_{\sqsubseteq}}, *, \diamond\right)$, with contraction constant, $k=1 / 2$.

From Example 5.3 we have that $\ell(\Phi(x) \sqcap \Phi(y)) \geq \ell(x \sqcap y)+1$ for all $x, y \in \Sigma^{\infty}$. So we have:

$$
\begin{aligned}
M_{d_{\sqsubseteq 1}}(\Phi(x), \Phi(y), t) & =1-2^{-\ell(\Phi x \sqcap \Phi y)} \\
& \geq 1-2^{-\ell(\Phi(x \sqcap y))}=1-2^{-(\ell(x \sqcap y)+1)} \\
& =1-\frac{1}{2} 2^{-\ell(x \sqcap y)}=1-\frac{1}{2}+\frac{1}{2} M_{d_{\sqsubseteq 1}}(x, y, t)
\end{aligned}
$$

for all $t>0$. 
Therefore $\Phi$ is a contraction on the bicomplete non-Archimedean ifqmspace $\left(\Sigma^{\infty}, M_{d_{\sqsubseteq 1}}, N_{d_{\sqsubseteq 1}}, *, \diamond\right)$ with contraction constant 1/2. So, by Theorem 8.13, $\Phi$ has a unique fixed point $z=z_{1} z_{2} \ldots$, which is obviously the unique solution to the recurrence equation $T$, i.e. $z_{1}=0$ and

$$
z_{n}=\frac{2(n-1)}{n}+\frac{n+1}{n} z_{n-1}
$$

for all $n \geq 2$. 


\section{Chapter 9}

\section{Contraction maps on fuzzy complexity spaces and expoDC algorithms}

\subsection{Introduction}

In Chapter 3 we discussed the complexity analysis of expoDC algorithms by using techniques of Denotational Semantics. To this end we constructed a suitable quasi-metric space, showing that the recurrence inequation associated to an expoDC algorithm gives rise to a contraction map on this quasi-metric space.

On the other hand, the extra general framework of fuzzy quasi-metric spaces provides extra information about the computational process because, in this context, the measurement of the distance from a "word" $x$ to another "word" $y$, automatically indicates if $x$ is a prefix of $y$ or not. In this chapter we discuss the complexity analysis of expoDC algorithms in the fuzzy quasi-metric context. This is done by showing that the recurrence inequation associated to an expoDC algorithm gives rise to a contraction map on a 
fuzzy quasi-metric space which is constructed here and whose elements are "complexity" functions of the function space $[0, \infty)^{\mathbb{N} \times \mathbb{N}}$. We prove that this contraction map has a unique fixed point, which, as in the construction given in Chapter 3, is the maximal element of the set of solutions of the recurrence inequation.

Let $d_{\sqsubseteq}$ be the Baire quasi-metric defined on $\Sigma^{\infty}$ (Example 5.3). It is well known that the quasi-metric space $\left(\Sigma^{\infty}, d_{\sqsubseteq}\right)$ is bicomplete. Let $\Sigma^{\omega}$ be the set of all infinite sequences ("words") over $\Sigma$. Then $\Sigma^{\omega}$ is a closed subset in $\left(\Sigma^{\infty}, d_{\sqsubseteq}^{s}\right)$. Indeed, let $w \in \Sigma^{\infty}$ and let $\left\{w_{n}\right\}_{n}$ be a sequence in $\Sigma^{\omega}$ such that $\lim _{n \rightarrow \infty} d_{\sqsubseteq}^{s}\left(w, w_{n}\right)=0$. Suppose that $\ell(w)<\infty$, then $d_{\sqsubseteq}^{s}\left(w, w_{n}\right) \geqslant 2^{-\ell(w)}$, which is a contradiction, hence $\ell(w)=\infty$, i.e. $w \in \Sigma^{\omega}$. So $\left(\Sigma^{\omega}, d_{\sqsubseteq}\right)$ is a bicomplete quasi-metric space.

Let $\mathcal{C}:=\{f: \mathbb{N} \times \mathbb{N} \rightarrow \Sigma\}$. We can define:

$$
\mathcal{C}_{m}:=\left\{\begin{array}{c}
f_{m}: \mathbb{N} \rightarrow \Sigma, \\
f_{m}(n)=f(m, n), \text { for each } m \in \mathbb{N}, \text { and } f \in \mathcal{C}
\end{array}\right\}
$$

It is clear that for each $m \in \mathbb{N}, \mathcal{C}_{m}$ can be identified with $\Sigma^{\omega}$, so, $\left(\mathcal{C}_{m}, d_{\sqsubseteq}\right)$ is a bicomplete quasi-metric space for each $m \in \mathbb{N}$.

Next we construct, for each $m \in \mathbb{N}$ the function $M_{m}$ on $\mathcal{C}_{m} \times \mathcal{C}_{m}$, given in the following way:

$$
\begin{aligned}
& M_{m}\left(f_{m}, g_{m}, 0\right)=0 \quad \text { for all } f_{m}, g_{m} \in \mathcal{C}_{m}, \\
& M_{m}\left(f_{m}, g_{m}, t\right)=1 \quad \text { if } f_{m} \text { is a prefix of } g_{m}, \text { and } t>0, \\
& M_{m}\left(f_{m}, g_{m}, t\right)=1-d_{\sqsubseteq}\left(f_{m}(n), g_{m}(n)\right)=1-2^{-\ell\left(f_{m} \sqcap g_{m}\right)} \quad \text { if } f_{m} \text { is not }
\end{aligned}
$$

a prefix of $g_{m}$, and $t>0$.

Then, by Proposition $5.4\left(\mathcal{C}_{m}, M_{m}, \wedge\right)$ is a G-bicomplete non-Archimedean 
fuzzy quasi-metric space.

We can define $M_{m}$ on $\mathcal{C} \times \mathcal{C}$ in the following way:

$$
M_{m}(f, g, t)=M_{m}\left(f_{m}, g_{m}, t\right)
$$

It is clear that

$$
M_{m}\left(f_{m}, g_{m}, t\right) \geq M_{m}\left(f_{m}, h_{m}, t\right) \wedge M_{m}\left(h_{m}, g_{m}, t\right)
$$

We define $M: \mathcal{C} \times \mathcal{C} \times[0, \infty) \rightarrow[0,1]$ as follows:

$$
\begin{aligned}
M(f, g, 0) & =0 \\
M(f, g, t) & =\inf _{m>n} M_{m}(f, g), t \in(n, n+1], n \in w
\end{aligned}
$$

for all $f, g \in \mathcal{C}$ and $t>0$. Then, we have the following theorem:

Theorem 9.1. $(\mathcal{C}, M, \wedge)$ is a non-Archimedean fuzzy quasi-metric space.

Proof. It is obvious that $M$ is a fuzzy set on $\mathcal{C} \times \mathcal{C} \times[0, \infty)$ which satisfies the following conditions for all $f, g \in \mathcal{C}$ :

$M(f, g, 0)=0$

$M(f, g, t)=M(g, f, t)=1$ for all $t>0$ if and only if $f=g$,

$M\left(f, g,_{-}\right)$is left continuous.

Let us see that:

$$
M(f, g, t+s) \geq M(f, h, t) \wedge M(h, g, s)
$$

Indeed, let $t \in(l, l+1], s \in(p, p+1]$ and $t+s \in(l+p+1, l+p+2]$, then we have: 


$$
\begin{aligned}
M(f, g, t+s) & =\inf _{m>l+p+1} M_{m}(f, g) \geq \inf _{m>l+p+1}\left(M_{m}(f, h) \wedge M_{m}(h, g)\right) \\
& =\inf _{m>l+p+1} M_{m}(f, h) \wedge \inf _{m>l+p+1} M_{m}(h, g),
\end{aligned}
$$

therefore

$$
\begin{aligned}
M(f, g, t+s) & \geq \inf _{m>l+p+1} M_{m}(f, h) \wedge \inf _{m>l+p+1} M_{m}(h, g) \\
& \geq \inf _{m>l} M_{m}(f, h) \wedge \inf _{m>p} M_{m}(h, g) \\
& =M(f, h, t) \wedge M(h, g, s) .
\end{aligned}
$$

Note that:

$$
\begin{aligned}
M(f, g, t+s) & \geq \inf _{m>l+p+1} M_{m}(f, h) \wedge \inf _{m>l+p+1} M_{m}(h, g) \\
& =\min \{M(f, h, t+s), M(h, g, t+s)\}
\end{aligned}
$$

for all $f, g \in \mathcal{C}$ and $t+s>0$, so $(\mathcal{C}, M, \wedge)$ is a non-Archimedean fuzzy quasimetric space.

It is well known that if $\left(\mathcal{C}_{m}, d_{\sqsubseteq}\right)$ is a bicomplete quasi-metric space, for each $m \in \mathbb{N}$, then $\left(\mathcal{C}, d_{s}\right)$ is a bicomplete quasi-metric space, where

$$
d_{s}(f, g)=\sup _{m \in \mathbb{N}} d_{\sqsubseteq}\left(f_{m}, g_{m}\right) .
$$

So, by Proposition $5.1\left(\mathcal{C}, M_{d_{s} 1}, \wedge\right)$ is a G-bicomplete non-Archimedean fuzzy quasi-metric space. It easy to see that $M(f, g, t)=M_{d_{s} 1}(f, g, t)$ if $t \in$ $(0,1]$, so (see Remark 2.1) the topoligies $\tau_{M}$ and $\tau_{M_{d_{s} 1}}$ are the same. Moreover $(\mathcal{C}, M, \wedge)$ is G-bicomplete if and only if $\left(\mathcal{C}, M_{d_{s} 1}, \wedge\right)$ is G-bicomplete. Hence, $(\mathcal{C}, M, \wedge)$ is a $\mathrm{G}$-bicomplete non-Archimedean fuzzy quasi-metric space. 


\subsection{Complexity fuzzy quasi-metric spaces and expoDC algorithms}

Following Remak 2.1 and Theorem 7.3 we present the following theorem.

Theorem 9.2. Let $(X, M, *)$ be a G-bicomplete fuzzy quasi-metric space. If $T$ is a self-map on $X$ such that there is $k \in(0,1)$ satisfying

$$
M(T x, T y, t) \geqslant 1-k+k M(x, y, t)
$$

for all $x, y \in X$ and $t \in(0, \varepsilon)$, with $\varepsilon>0$, then $f$ has a unique fixed point.

We shall prove that the recurrence inequation associated to an expoDC algorithm gives rise to a contraction map on $(\mathcal{C}, M, \wedge)$ in the sense of Theorem 9.2, so the contraction map has a unique fixed point, and then the complexity of the algorithm is represented via this fixed point.

Example 9.1. Let $\Phi: \mathcal{C} \rightarrow \mathcal{C}$ be the functional associated to the recurrence inequation of the complexity analysis of expoDC algorithm (see Section 3.4). Next we show that $\Phi$ is a contraction (in the sense of Theorem 9.2) on the G-bicomplete non-Archimedean fuzzy quasi-metric space $(\mathcal{C}, M, \wedge)$, with contraction constant $1 / 2$.

To this end, we first note that, by construction, given $m \in \mathbb{N}$, we have $\ell\left(\Phi\left(f_{m}\right)\right) \geq \ell\left(f_{m}\right)+1$ for all $f_{m} \in \mathcal{C}_{m}$ (in particular, $\ell\left(\Phi\left(f_{m}\right)\right)=\infty$ whenever $\left.\ell\left(f_{m}\right)=\infty\right)$.

Furthermore, it is clear that

$$
f_{m} \sqsubseteq g_{m} \Longleftrightarrow \Phi\left(f_{m}\right) \sqsubseteq \Phi\left(g_{m}\right)
$$

and consequently

$$
\Phi\left(f_{m} \sqcap g_{m}\right) \sqsubseteq \Phi\left(f_{m}\right) \sqcap \Phi\left(g_{m}\right)
$$


for all $f_{m}, g_{m} \in \mathcal{C}_{m}$. Hence

$$
\ell\left(\Phi\left(f_{m}\right) \sqcap \Phi\left(g_{m}\right)\right) \geq \ell\left(\Phi\left(f_{m} \sqcap g_{m}\right)\right) \geq \ell\left(f_{m} \sqcap g_{m}\right)+1
$$

for all $f_{m}, g_{m} \in \mathcal{C}_{m}$.

For each $m \in \mathbb{N}$ we have:

$$
\begin{aligned}
M_{m}(\Phi f, \Phi g, t) & =M_{m}\left(\Phi f_{m}, \Phi g_{m}, t\right)=1-2^{-\ell\left(\Phi f_{m} \sqcap \Phi g_{m}\right)} \\
& \geq 1-2^{-\ell\left(\Phi\left(f_{m} \sqcap g_{m}\right)\right)} \geq 1-2^{-\left(\ell\left(f_{m} \sqcap g_{m}\right)+1\right)} \\
& =1-\frac{1}{2} 2^{-\ell\left(f_{m} \sqcap g_{m}\right)} \\
& =1-\frac{1}{2}+\frac{1}{2} M_{m}(f, g, t)
\end{aligned}
$$

for all $f, g \in \mathcal{C}$.

So, we have:

$$
\begin{aligned}
M(\Phi f, \Phi g, t) & =\inf _{m \geq 1} M_{m}(\Phi f, \Phi g, t) \geq \inf _{m \geq 1}\left(1-\frac{1}{2}+\frac{1}{2} M_{m}(f, g, t)\right) \\
& =1-\frac{1}{2}+\frac{1}{2} M(f, g, t)
\end{aligned}
$$

for all $t \in(0, \varepsilon)$, with $\varepsilon>0$, and for all $f, g \in \mathcal{C}$. Hence $\Phi$ is a contraction on the G-bicomplete non-Archimedean fuzzy quasi-metric space $(\mathcal{C}, M, \wedge)$, with contraction constant $1 / 2$, and the conditions of Theorem 9.2 are satisfied, therefore $\Phi$ has a unique fixed point $f_{0}$.

Finally we claim that $f_{0}$ is the maximal element of the set of solutions of the recurrence inequation associated to expoDC algorithm (Section 3.4). Indeed, by construction if $g$ satisfies this inequation then $\Phi g$ satisfies this inequation too, so we have 


$$
g \leq \Phi g \leq \Phi \Phi g \leq \ldots \leq \Phi^{n} g
$$

so

$$
g \leq \lim _{n \rightarrow \infty} \Phi^{n} g=f_{0}
$$

i.e

$$
g \leq f_{0}
$$




\section{Bibliography}

[1] A.V. Aho, J.E. Hopcroft, J.D. Ullman, Data Structures and Algorithms, Addison-Wesley Publishing Company, Massachusetts.1983.

[2] C. Alaca, D. Turkoglu, C. Yildiz, Fixed points in intuitionistic fuzzy metric spaces, Chaos, Solitons \& Fractals 29 (2006), 1073-1078.

[3] J.W. de Bakker, E.P. de Vink, A metric approach to control flow semantics, in: Proc. Eleventh Summer Conference on General Topology and Applications, Ann. New York Acad. Sci. 806 (1996), 11-27.

[4] J.W. de Bakker, E.P. de Vink, Denotational models for programming languages: applications of Banach's fixed point theorem, Topology Appl. 85 (1998), 35-52.

[5] J.W. de Bakker, E.P. de Vink, Control Flow Semantics, The MIT Press, Cambridge, Massachusetts, 1996.

[6] G. Brassard, P. Bratley, Fundamentals of Algorithms, Prentice Hall, 1996.

[7] M. Davis, E. Weyuker, Computability, Complexity and Languages, New York Academic Press, 1983.

[8] R.V. Demico, G.J. Klir, Fuzzy logic in geology, Elsevier Academic Press, 2004. 
[9] M.S. El Naschie, On the uncertainly of Cantorian geometry and two-slit experiment, Chaos, Solitons \& Fractals 9 (1998), 517-529.

[10] M.S. El Naschie, On the verification of heterotic strings theory and $\epsilon^{(\infty)}$ theory, Chaos, Solitons \& Fractals 11 (2000), 2397-2408.

[11] M.S. El Naschie, The two-slit experiment as the foundation of E-infinity of high energy physics, Chaos, Solitons \& Fractals 25 (2005), 509-514.

[12] M.S. El Naschie, 't Hooft ultimate building blocks and space-time as an infinite dimensional set of transfinite discrete points, Chaos, Solitons \& Fractals 25 (2005), 521-524.

[13] M.S. El Naschie, Non-Euclidean spacetime structure and the two-slit experiment, Chaos, Solitons \& Fractals 26 (2005), 1-6.

[14] R. Engelking, General Topology, PWN-Polish Sci. Publ., Warsaw, 1977.

[15] L. Fan, A new approach to quantitative domain theory, Electronic Notes Theoret. 45 (2001), URL: htt://www.elsevier.nl/locate/entcs/volume45.html 11 pages.

[16] P. Flajolet, Analytic analysis of algorithms, in: 19th Internat. Colloq. ICALP'92, Vienna, July 1992; Automata, Languages and Programming, Lecture Notes in Computer Science 623, W. Kuich editor (1992), pp. $186-210$.

[17] P. Fletcher, W.F. Lindgren, Quasi-Uniform Spaces, Marcel Dekker, New York, 1982.

[18] L.M. García-Raffi, S. Romaguera, E.A. Sánchez-Pérez, Sequence spaces and asymmetric norms in the theory of computational complexity, Math. Comput. Model. 36 (2002), 1-11. 
[19] A. George, P. Veeramani, On some results in fuzzy metric spaces, Fuzzy Sets and Systems 64 (1994), 395-399.

[20] A. George, P. Veeramani, On some results of analysis of fuzzy metric spaces, Fuzzy Sets and Systems 90 (1997), 365-368.

[21] M. Grabiec, Fixed points in fuzzy metric spaces, Fuzzy Sets and Systems 27 (1988), 385-389.

[22] V. Gregori, S. Romaguera, Some Properties of Fuzzy Metric Spaces, Fuzzy Sets and Systems 115 (2000), 485-489.

[23] V. Gregori, S. Romaguera, On Completion of Fuzzy Metric Spaces, Fuzzy Sets and Systems 130 (2002), 399-404.

[24] V. Gregori, S. Romaguera, Fuzzy quasi-metric spaces, Appl. Gen. Topology 5 (2004), 129-136.

[25] V. Gregori, S. Romaguera, P. Veeramani, A note on intuitionistic fuzzy metric spaces, Chaos, Solitons \& Fractals 28 (2006), 902-905.

[26] V. Gregori, A. Sapena, On fixed point theorems in fuzzy metric spaces, Fuzzy Sets and Systems 125 (2002), 245-253.

[27] P. Hàjek, Metamathematics of Fuzzy Logic Kluwer, Dordrecht, 1998

[28] P. Hàjek, Basic fuzzy and BL-algebras, Soft Computing 2 (1998) 124128.

[29] G. Kahn, The semantics of a simple language for parallel processing, in: Proc. IFIP Congresss, Elsevier and North-Holland, Amsterdam 1974, pp. $471-475$.

[30] I. Kramosil, J. Michalek, Fuzzy metrics and statistical metric spaces, Kybernetika 11 (1975), 336-344. 
[31] R. Kruse, Data Structures and Program Design, Prentice-Hall, Inc., 1984.

[32] H.P.A. Künzi, Nonsymmetric topology, in: Proc. Colloquium on Topology, Szekszárd, Hungary, 1993, Colloq. Math. Soc. János Bolyai Math. Studies 4 (1995), 303-338.

[33] H.P.A. Künzi, Nonsymmetric distances and their associated topologies: About the origins of basic ideas in the area of asymmetric topology, in: C.E. Aull, R. Lowen (Eds.), Handbook of the History of General Topology, vol. 3, Kluwer, Drodrecht, 2001, pp. 853-968.

[34] S.G. Matthews, Partial metric topology, in: Proc. 8th Summer Conference on General Topology and Applications, Ann. New York Acad. Sci. 728 (1994), 183-197.

[35] D. Mihet, A Banach contraction theorem in fuzzy metric spaces, Fuzzy Sets and Systems 144 (2004), 431-439.

[36] J.H. Park, Intuitionistic fuzzy metric spaces, Chaos, Solitons \& Fractals 22 (2004), 1039-1046.

[37] J. Rodríguez-López, S. Romaguera, A. Sapena, Casi-métricas difusas y dominios de computación; aceptado para su publicación en Rev. Iberoamericana de Sistemas, Cibernética e Informática. Publicación previa en: Memorias CISCI 2004, vol. II, pp. 388-391.

[38] J. Rodríguez-López, S. Romaguera, O.Valero, Asympotitc complexity of algorithms via the nonsymmetric Hausdorff distance, Computing Letters 2 (2006), 155-161.

[39] J. Rodríguez-López, S. Romaguera, O.Valero, Denotational semantics for programming languages, balanced quasi-metrics and fixed points, Internat. J. Comput. Math., to appear. 
[40] S. Romaguera, E.A. Sánchez-Pérez, O. Valero, Computing complexity distances between algorithms, Kybernetika 39 (2003), 569-582.

[41] S. Romaguera, A. Sapena, P. Tirado, The Banach fixed point theorem in fuzzy quasi-metric spaces with application to the domain of words, Topology Appl 154 (2007), 2196-2203.

[42] S. Romaguera, M. Schellekens, Quasi-metric properties of complexity spaces, Topology Appl., Vol. 98, 1999, pp. 311-322.

[43] S. Romaguera, M. Schellekens, Duality and quasi-normability for complexity spaces, Appl. Gen. Topology 3 (2002), 91-112.569-582.

[44] S. Romaguera, M.P. Schellekens, P. Tirado, O. Valero, Contraction maps on complexity spaces and expoDC algorithms, Proceedings ICCMSE 2007, American Institute of Physics, (AIP), Mathematical and Statistical Physics Subseries, Vol. II, pp. 1343-1346.

[45] S. Romaguera, P. Tirado, Complexity fuzzy quasi-metric spaces. Univ. Politécnica de Valencia, preprint.

[46] S. Romaguera, P. Tirado, Funciones de Contracción Difusa y Métodos de Punto Fijo Aplicados al Análisis de Complejidad Algorítmica. Memorias CISCI 2005, Vol. I, pp. 339-342.

[47] S. Romaguera, P. Tirado, On fixed point theorems in intuitionistic fuzzy metric spaces, Internat. J. Nonlinear Sci. Numer. Simul., 8 (2007), 233238.

[48] S. Romaguera, P. Tirado, Contraction maps on ifqm-spaces with application to recurrence equations of Quicksort, In: Proc. MFCSIT 06, Electronic Notes Theoret. Comput. Sci., to appear.

[49] A. Sapena, A contribution to the study of fuzzy metric spaces, Appl. Gen. Topology 2 (2001), 63-76. 
[50] M. Schellekens, The Smyth completion: a common foundation for denonational semantics and complexity analysis, in: Proc. MFPS 11, Electronic Notes in Theoretical Computer Science 1 (1995), 211-232.

[51] M. Schellekens, Complexity domains. A relative view of complexity, manuscript.

[52] M.P. Schellekens, A characterization of partial metrizability. Domains are quantifiable, Theoret. Comput. Sci. 305 (2003), 409-432.

[53] M.P. Schellekens, The correspondence between partial metrics and semivaluations, Theoret. Comp. Sci. 315 (2004), 135-149.

[54] V.M. Sehgal, A.T. Bharucha-Reid, Fixed points of contraction mappings on PM-spaces, Math. Systems Theory 6 (1972), 97-100.

[55] B. Schweizer, A. Sklar, Statistical metric spaces, Pacific J. Math. 10 (1960), 314-334.

[56] B. Schweizer, A. Sklar, Probabilistic metric spaces, North-Holland, Amsterdam, 1983.

[57] M.B. Smyth, Quasi-uniformities: Reconciling domains with metric spaces, in: Mathematical Foundations of Programming Langauge Semantics, 3rd Workshop, Tulane 1987, Lecture Notes Computer Science, vol. 298, M. Main et al (eds.), Springer, Berlin, 1988, 236-253.

[58] P. Tirado, Algunas observaciones sobre la topología de los espacios métricos difusos , Memorias XIII Encuentro de Topología, Castro Urdiales, Spain, 2006.

[59] R. Vasuki, P. Veeramani, Fixed point theorems and Cauchy sequences in fuzzy metric spaces, Fuzzy Sets and Systems 135 (2003), 415-417. 
[60] Q. Zhang, L. Fan, Continuity in quantitative domains, Fuzzy Sets and Systems 154 (2005) 118-131. 